\title{
Directional radiometry and radiative transfer: The convoluted path from centuries-old phenomenology to physical optics
}

\author{
Michael I. Mishchenko* \\ NASA Goddard Institute for Space Studies, 2880 Broadway, New York, NY 10025, USA
}

\section{A R T I C L E I N F O}

Article history:

Received 18 February 2014

Received in revised form

26 February 2014

Accepted 28 February 2014

Available online 13 March 2014

Keywords:

Electromagnetic scattering

Physical optics

Directional radiometry

Radiative transfer

Macroscopic Maxwell equations

Mesoscopic physics

\begin{abstract}
A B S T R A C T
This Essay traces the centuries-long history of the phenomenological disciplines of directional radiometry and radiative transfer in turbid media, discusses their fundamental weaknesses, and outlines the convoluted process of their conversion into legitimate branches of physical optics.
\end{abstract}

Published by Elsevier Ltd.

\section{Introduction}

It is a great honor to have become the second recipient of the Hendrik C. Van de Hulst Award presented by Elsevier in the general category of Electromagnetic Scattering. It is also a special pleasure for me to receive this award following Prof. Joop W. Hovenier (Fig. 1) who has always exerted strong influence on my research and had unknowingly served as an implicit adviser during my $\mathrm{PhD}$ studies and early years in science. Part of the award ceremony was a Van de Hulst Lecture presented at the

\footnotetext{
Abbreviations: CCD, charge-coupled device; DTO, dyadic transition operator; ELS, Electromagnetic and Light Scattering; FEs, Foldy equations; GISS, Goddard Institute for Space Studies; JQSRT, Journal of Quantitative Spectroscopy and Radiative Transfer; MMEs, macroscopic Maxwell equations; NASA, National Aeronautics and Space Administration of the USA; PST, Poytning-Stokes tensor; QED, quantum electrodynamics; RTE, radiative transfer equation; RTT, radiative transfer theory; VIE, volume integral equation; WCR, well-collimated radiometer

*Tel.: +1 212678 5590; fax: +12126785222.

E-mail address: crmim2@gmail.com
}

14th Conference on Electromagnetic and Light Scattering (ELS) on 20 June 2013. Another official part of this award is the honor and obligation to publish in the Journal of Quantitative Spectroscopy and Radiative Transfer (JQSRT) a scientific Essay intended to summarize the recipient's personal view of the state-of-the-art of one or more disciplines related to electromagnetic scattering by particles and particulate media. Needless to say, these disciplines are expected to have been foci of the recipient's own research leading to the Van de Hulst Award.

The formats and styles of the Van de Hulst Lecture and the Van de Hulst Essay are still in a state of flux since only two such lectures have been presented and only one such Essay has been published [1]. On one hand, this lack of established traditions appears to represent a challenge. Yet on the other hand I feel that it sets me free to adopt an ad hoc format for this Essay and essentially speak my mind with the hope that the result will be instructive to the JQSRT readership. Therefore, I have decided to focus on the current state of the disciplines of directional radiometry and radiative transfer as I understand them following 


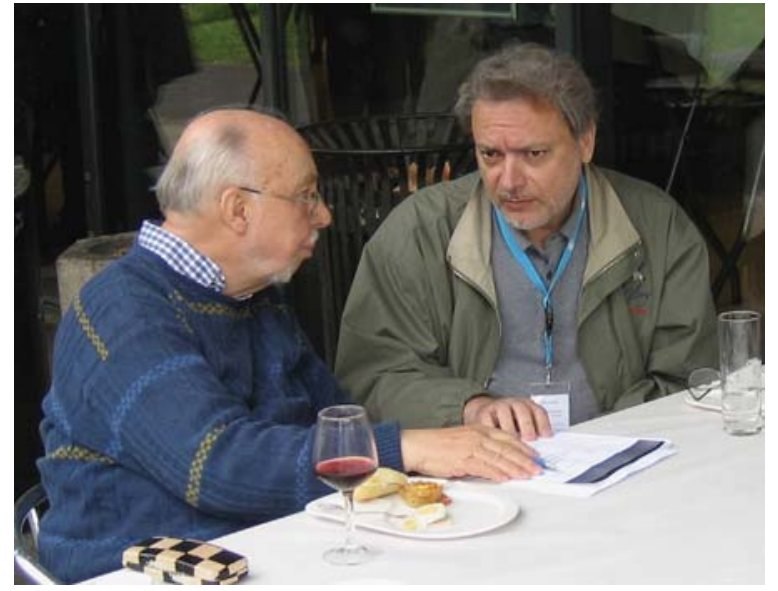

Fig. 1. Joop Hovenier (left) and Michael Mishchenko at ELS-XIV in Lille on 17 June 2013.

almost 30 years of research. More specifically, this Essay will largely be devoted to the centuries-long history of the phenomenological stage of these disciplines which, intriguingly, is not over yet, followed by the history of the uneasy process that has ultimately led both disciplines to become full-fledged branches of physical optics.

Although Van de Hulst's classical treatises on light scattering [2] and radiative transfer [3] have been essential sources of my knowledge of both disciplines, I have had the privilege of meeting him in person only twice. The first meeting occurred in Leningrad (now St. Petersburg) in October of 1990 during a Symposium dedicated to the 100th anniversary of the radiative transfer equation (RTE). That Symposium was organized by Academician Viktor Sobolev and Professor Vsevolod Ivanov and served to assemble many members of the renowned Soviet school of radiative transfer (Fig. 2). Professor Van de Hulst (Fig. 3) was a special invitee and a de facto ambassador of the western school. The majority of presentations were given in Russian, and so during two sessions I was asked by Vsevolod Ivanov to translate the talks into English personally for Professor Van de Hulst. That task of speaking so much English for the first time in my life turned out to be a challenge, and by the middle of the second session my tongue and lips started to fail. Professor Van de Hulst did notice that and with a soft smile told me not to worry since most formulas required no translation. I was also asked to accompany Professor Van de Hulst on his commutes between Pulkovo (the location of the famous Pulkovo Astronomical Observatory) and Petrodvorets (the location of the Astronomy Department of the Leningrad University). One of these trips coincided with a beautiful sunset which we could observe through the train window; looking at splendid colors of that sunset naturally triggered an instructive discussion of various atmospheric optical phenomena.

Our second meeting occurred during the inaugural Conference on Light Scattering by Non-Spherical particles in May of 1995. That event was organized by Joop Hovenier at the Free University of Amsterdam [4] and served to initiate the prominent series of ELS conferences.
The 1998 ELS conference was convened at the NASA Goddard Institute for Space Studies (GISS) in New York, and Professor Van de Hulst had kindly agreed to open it with a keynote lecture. He was very enthusiastic about the occasion to visit the place where he spent a six-month sabbatical in 1962, the result of which was his famous NASA report on the adding/doubling method [5]. Unfortunately, just two days before the opening of the conference Professor Van de Hulst faxed me with an apology for being unable to come to New York because of certain health issues. However, he was still able to contribute the instructive Foreword [6] to the monograph on light scattering by nonspherical particles [7] which has proved to be an important collective outcome of the New York conference.

Since Joop Hovenier did his PhD work under the supervision of Professor Van de Hulst and since my early research had benefitted so much from Joop's publications (especially the 1983 review [8] co-authored by Cornelis van der Mee), I consider myself one of Van de Hulst's "indirect" disciples. My direct scientific genealogy can be traced to Academician Viktor Ambartsumian, one of the founders of theoretical astrophysics. Indeed, I did my PhD work under the supervision of Dr. Edgard Yanovitskij whose PhD thesis was in turn supervised by Academician Sobolev (Fig. 4), the prominent Soviet astrophysicist and the best known PhD student of Academician Ambartsumian (Fig. 5). My early research was also influenced by two other members of the Ambartsumian-Sobolev school of radiative transfer, Vsevolod Ivanov and Helmut Domke (Fig. 6). Furthermore, Vsevolod Ivanov served as an Official Opponent on my PhD and Habilitation thesis defenses.

As already mentioned, I owe my initial basic knowledge of the theory of radiative transfer to Van de Hulst's monograph [3], as well as to the so-called "blue Sobolev" [9]. ${ }^{1}$ That nickname refers to the navy blue color of the cover in the original Russian edition of this well-known monograph and was used by Soviet scientists to distinguish it casually from the "black Sobolev" [11]. As a novice in the field of radiative transfer, I took for granted the apparent simplicity and obviousness of the main phenomenological concepts of this discipline and for some time had not realized that the "traditional" radiative transfer theory (RTT) is, figuratively speaking, a "colossus with feet of clay". My awakening started in 1986 when I read the Russian edition of the 1978 book by Ishimaru [12]. It was quite surprising to learn that

\footnotetext{
${ }^{1}$ Another essential source was the famous review by my future colleagues at GISS James Hansen and Larry Travis [10]. This paper was immensely popular among my Soviet colleagues despite the fact that only a few preprints and even fewer reprints were in circulation. I still vividly remember how upset Edgard Yanovitskij became when he found that his copy of the preprint had been stolen from his desk. While being unethical, this was obviously an act of desperation on someone's part as well as an implicit compliment to a great publication. In 1985, the first year of my PhD studies, it took a bit of courage to send a postcard to Larry Travis asking for a reprint. I thought that I had no chance since 11 years after the publication of the paper all reprints were likely to be gone; furthermore, I did not know the street address (it was not included as part of the authors' affiliation) and sent the postcard to NASA GISS, New York, NY 10025, USA. To everyone's (and my own) surprise, two months later I became a proud owner of an original reprint. Needless to say, I never left it on my desk unattended.
} 

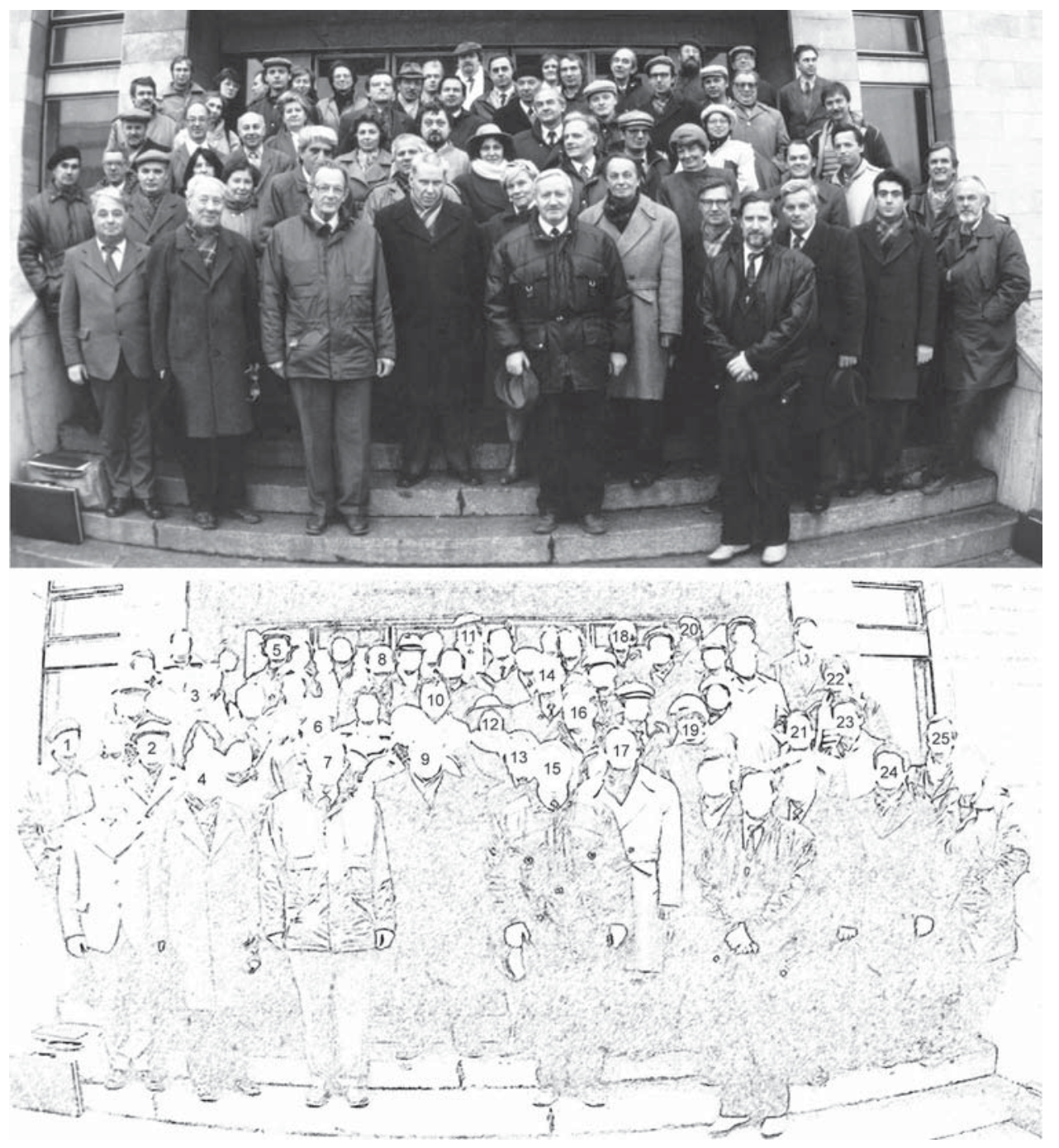

Fig. 2. Participants of the Leningrad Symposium dedicated to the 100th anniversary of the radiative transfer equation (October 1990). From left to right: Roman Kostyk (1), Viktor Loskutov (2), Dmitry Nagirner (3), Kusiel Shifrin (4), Michael Mishchenko (5), Arthur Nikoghossian (6), Hendrik Van de Hulst (7), Alexander Kokhanovsky (8), Viktor Sobolev (9), Nikoli Konovalov (10), Aavo Heinlo (11), Tamara Sushkevich (12), Tatyana Germogenova (13), Arkady Ivanov (14), Igor Minin (15), Vsevolod Ivanov (16), Yuri Gnedin (17), Edgard Yanovitskij (18), Zhanna Dlugach (19), Nikolai Rogovtsov (20), Aleksandr Kolesov (21), Nikolai Voshchinnikov (22), Vladimir Grinin (23), Hovannes Pikichian (24), and Tõnu Viik (25).

there appeared to be at least two different and completely unrelated ways of arriving at exactly the same scalar RTE for a turbid medium, viz., the "traditional" back-of-an-envelope derivation allegedly based on the concept of radiance and energy conservation considerations as well as the one allegedly based on the scalar wave theory. As someone with a bit of a background in mathematical logic, I thought that if that were the case then one of the derivations must be a direct corollary of the other because otherwise it would be a fake derivation essentially amounting to the postulation of the RTE based on verbal "simple physical considerations".

The following 25 years of study and research have confirmed that initial suspicion. Furthermore, I believe that this research has served to conclude the work initiated by Rudolph Preisendorfer, Yuri Barabanenkov, Anatoli Borovoi, Yuri Gnedin, Emil Wolf, Akira Ishimaru, Leung Tsang and others in that it has brought the disciplines of directional radiometry and radiative transfer into the realm of physical optics. Therefore, what follows is a personal and, by definition, subjective account of what it has taken to finally convert the centuries-old phenomenologies of directional radiometry and radiative transfer, as applied to particulate media, into first-principle theories.

\section{Phenomenology}

A thorough account of the early history of directional photometry $^{2}$ was provided by DiLaura in the introduction to Ref. [13]. He attributed the culmination of medieval optics to

\footnotetext{
${ }^{2}$ For the purposes of this Essay, the terms "photometry" and "radiometry" will be used interchangeably.
} 


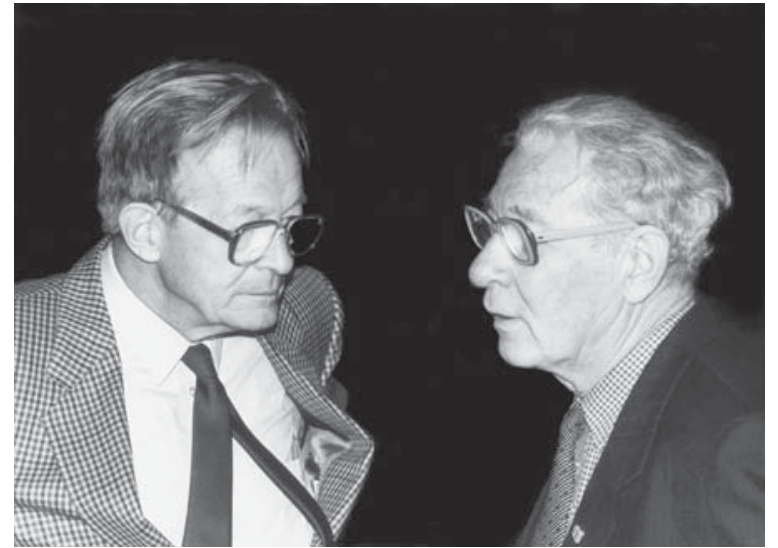

Fig. 3. Hendrik Van de Hulst (left) and Kusiel Shifrin at the Leningrad Symposium dedicated to the 100th anniversary of the radiative transfer equation (October 1990).

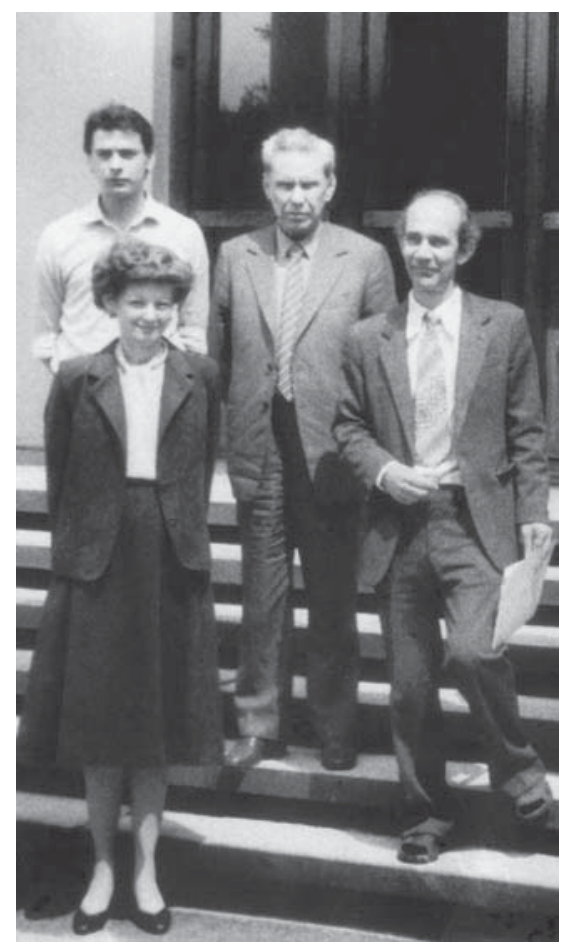

Fig. 4. From left to right: Michael Mishchenko, Zhanna Dlugach, Viktor Sobolev, and Edgard Yanovitskij (Kyiv, June 1985).

Ad Vitellionem Paralipomena [14] by Johannes Kepler (15711630; Fig. 7) published in 1604 and containing one of the most fundamental elements of photometry, viz., the attenuation of the intensity of light as the inverse square of distance from a point-like source. However, establishing photometry as a scientific discipline was the outcome of systematic studies by the French natural scientist and engineer Pierre Bouguer (1698-1758) followed by those of the Swiss mathematician, natural scientist, and philosopher Johann Lambert (17281777).

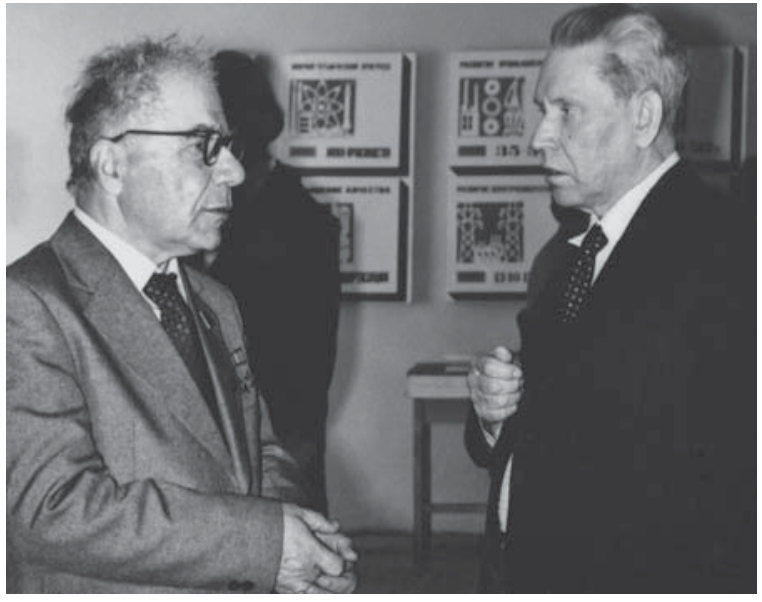

Fig. 5. Academicians Viktor Ambartsumian (left) and Viktor Sobolev (Leningrad, May 1980).

Bouguer's Essai d'Optique [15] was published in 1729, while its thorough augmentation, Traité d'Optique [16] (Fig. 8), appeared posthumously in 1760. Bouguer's research was mostly experimental and relied on several ingeniously designed photometric instruments. He was the first to realize that the human eye cannot be used as an accurate absolute meter of brightness but is quite capable of establishing the equality of brightness of two adjacent surfaces. In Essai d'Optique Bouguer describes the use of Kepler's law of inverse squares and the human eye as an equality indicator to derive the ratio of luminous intensities of two light sources and discovers the famous exponential attenuation law ${ }^{3}$ by studying the diminution of light as it passes through translucent media (Fig. 9). He also analyzed the reflection of light by rough surfaces and proposed the idea of modeling such diffuse reflectors as consisting of small randomly oriented mirrors whose orientation distribution would determine the macroscopic angular reflectance. In his Photometria [17] (Fig. 8), Lambert was the first to extensively and systematically use contemporary mathematics, including calculus, to interpret experimental results and developed the mathematical foundation of radiometry by introducing specific definitions of photometric quantities and a unified set of photometric principles and laws.

The impact of Bouguer's and Lambert's work was so profound that even now much of illumination engineering is based, directly or indirectly, on their treatises. Perhaps the only significant augmentation dating from 1854 was the incorporation of the solution concentration into Bouguer's exponential attenuation law by August Beer (18251863) [18].

With the development of Maxwell's electromagnetics in 1864 and the realization that light consists of electromagnetic waves, the photometry of Bouguer and Lambert

\footnotetext{
${ }^{3}$ Bouguer's exponential attenuation law is often incorrectly attributed to Lambert. This is thoroughly inappropriate since Lambert had read Bouguer's Essai d'Optique published in 1729 and frequently cited it in his own Photometria [17] published in 1760.
} 


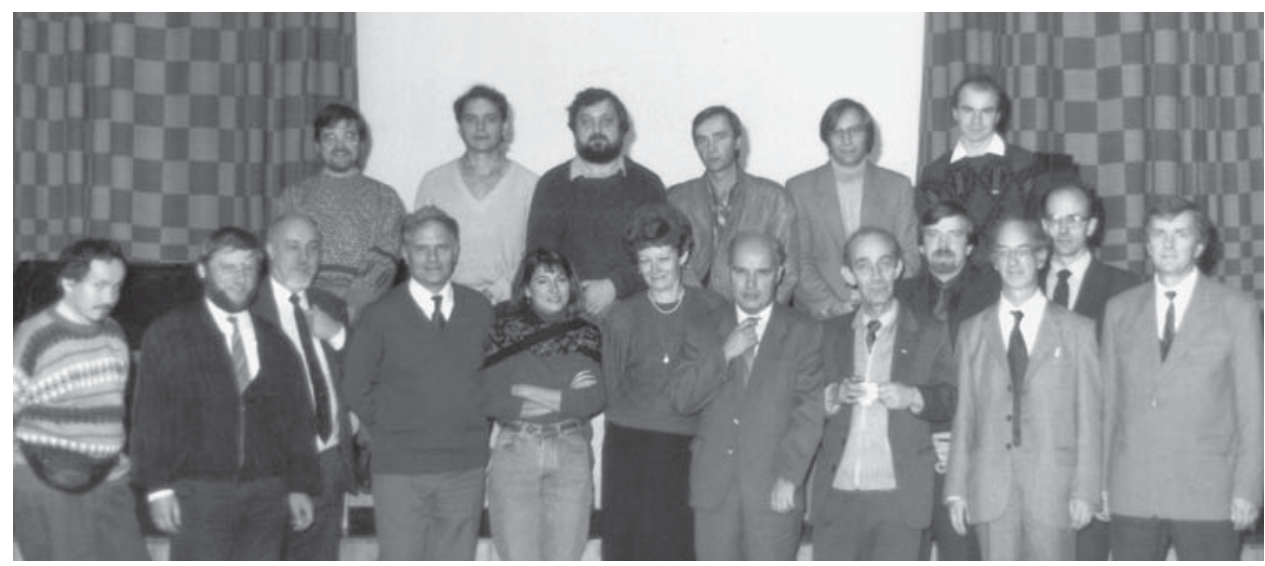

Fig. 6. Participants of the 2nd Conference on Polarized Radiative Transfer, Tõravere, Estonia, 17-18 October 1991. First row, from left to right: Cornelis van der Mee, Helmut Domke, Joop Hovenier, Vsevolod Ivanov, Marianne Faurobert-Scholl, Zhanna Dlugach, Viktor Loskutov, Edgard Yanovitskij, Aavo Heinlo, Dmitry Nagirner, Juris Freimanis, and Tõnu Viik. Second row, from left to right: Hans Scholl, Michael Mishchenko, Nikolai Konovalov, Eugene Ustinov, and Sergei Strelkov.

could no longer be considered a branch of physics based on first principles and had thus become a purely phenomenological discipline. ${ }^{4}$ However, this profound and irreversible shift in the conceptual status of the old directional photometry has largely been ignored. All in all, phenomenological photometry, as summarized eloquently in 1936 by Andrei Gershun (1903-1952) in terms of the so-called "light field" [21], has been one of the oldest surviving paradigms ${ }^{5}$ in contemporary science despite its whopping

\footnotetext{
${ }^{4}$ A physical theory is called phenomenological if it expresses mathematically the results of observed phenomena without clarifying their fundamental origin and significance. Typically, the development of a phenomenological theory is based on experience-based heuristic shortcuts lacking rigorous justification. Most phenomenological theories are short-lived and get replaced by fundamental first-principle theories. However, as we discuss in this Essay, some phenomenologies can survive for centuries despite their inherently limited scientific value and eventually become an impediment to scientific progress. The Latin term "Phenomenologia" was introduced by the German Lutheran theologian and theosopher Christoph Friedrich Oetinger in 1736 [19]. Subsequently, the German term "Phänomenologie" was used by Lambert to name his "doctrine of appearance" [20]. It should be noted that Lambert viewed his photometry as a phenomenological discipline from the very outset.

5 The concepts of scientific paradigms and paradigm shifts emerged in the framework of the historical approach in the philosophy of science according to which only by studying history of science can we gain an adequate understanding of human reason. These profound notions were introduced and analyzed, using different terminology, by the 20th century French philosophers Gaston Bachelard, Georges Canguilhem, and Michel Foucault, but the roots of their work can be traced to the older French tradition of science studies, going back to Auguste Comte and including later thinkers such as Pierre Duhem and Henri Poincaré (see, e.g., Refs. [22-26]). Thomas Kuhn [27] systematized and popularized these ideas and re-instituted the use of the term "paradigm" introduced by Plato in his dialog Timaeus to name the eternal pattern used by a divine Craftsman (the Demiurge) to create the universe. A universally accepted definition of a scientific paradigm hardly exists, but in general it can be summarized as a self-contained set of concepts, values, perceptions, and practices shared by a large scientific community; it forms a particular vision of reality and is the basis of the way the community organizes itself. A paradigm shift (or, in Kuhn's terminology, a scientific revolution) occurs when a given discipline switches from one distinct paradigm to another, often as a result of a long process.
}

disconnect from the "mainland" of modern physics (in the words of Rudolph Preisendorfer [28]).

The main contribution by the German physicist and mathematician Eugen von Lommel (1837-1899) was to introduce, in 1887, the notion of the amount of radiant energy crossing an imaginary geometrical rather than an actual physical surface element. This allowed him to conceptualize the directional flow of radiant energy through space and introduce the integral form of the RTE as a way of solving the problem of diffusion of light through a turbid medium composed of isotropically scattering centers [29] (Fig. 10). Virtually identical results were published independently by the Russian physicist Orest Khvolson (1852-1934) two years later ${ }^{6}$ [30] (Fig. 10; see also the instructive account of the early history of the phenomenological RTT by Ivanov [31]).

The work by Lommel and Khvolson has remained largely unnoticed. The first introduction of the RTE has traditionally been attributed to Arthur Schuster (18511934). In actuality, however, Schuster's paper of 1905 [32] contains what is now known as the two-stream approximation rather than the integral or integro-differential form of the RTE. The first phenomenological derivation of the RTE in the case of anisotropic scattering was given by Louis Vessot King in 1913 [33]. Like Lommel and Khvolson, King introduced the integral form of the RTE rather than the integro-differential equation that eventually assumed the status of being the canonical form of the RTE. Interestingly, the direct microphysical derivation from the Maxwell equations yields the integral form of the RTE, the integro-differential form being a corollary.

\footnotetext{
${ }^{6}$ The first page of Ref. [30] contains an interesting footnote by Academician $\mathrm{H}$. Wild according to which the paper was originally submitted in the fall of 1885 . It was then withdrawn by the author who hoped to obtain a more complete solution of the main equation. The paper was resubmitted in the fall of 1888 essentially in its original form. It thus appears that Lommel and Khvolson introduced the RTE independently of each other.
} 


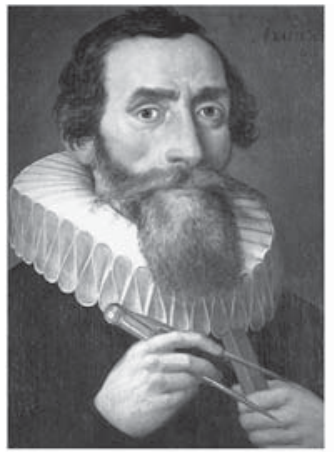

Johannes Kepler

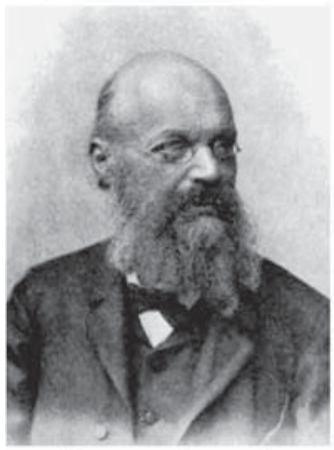

Eugen von Lommel

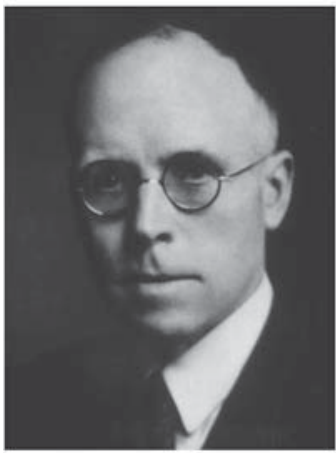

E. Arthur Milne

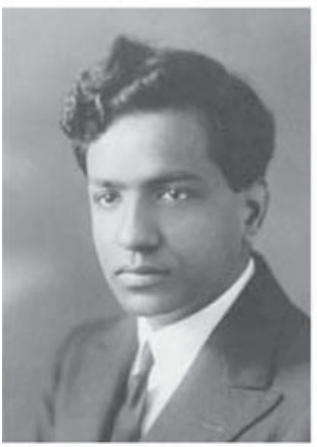

S. Chandrasekhar

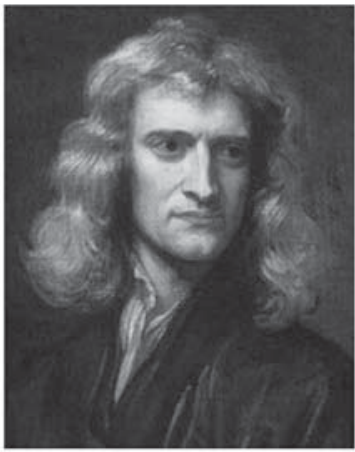

Isaac Newton

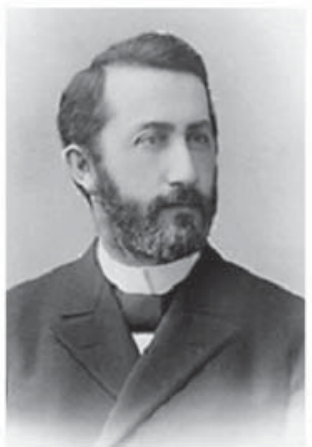

Orest Khvolson

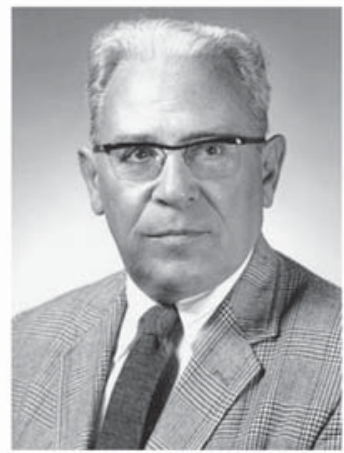

Eberhard Hopf

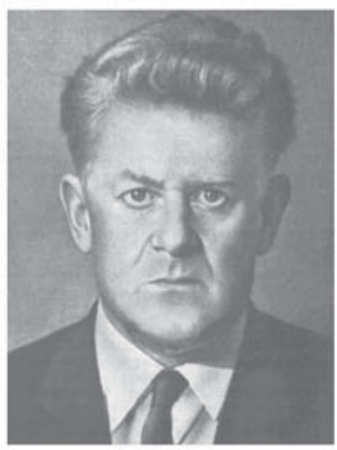

Georgi Rozenberg

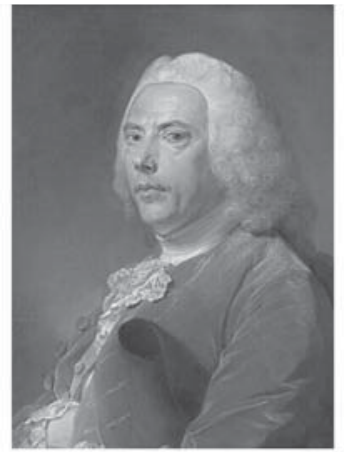

Pierre Bouguer

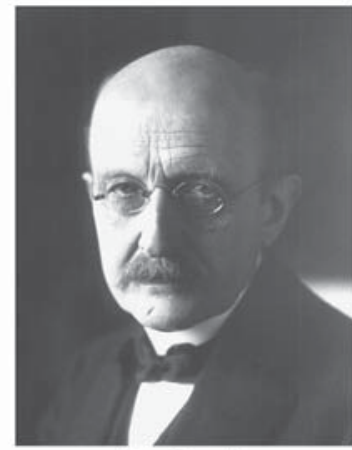

Max Planck

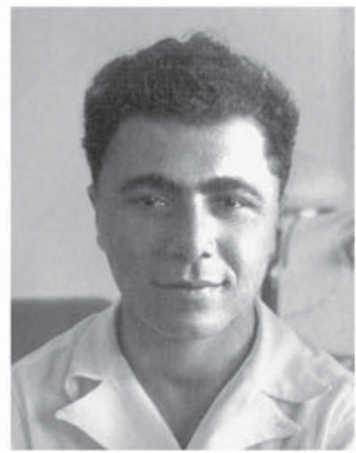

Viktor Ambartsumian

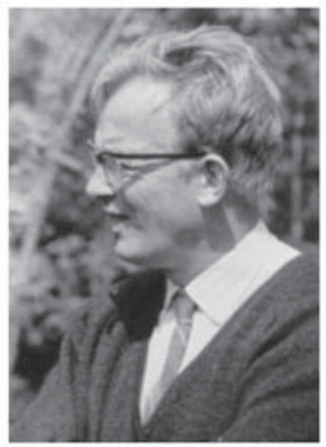

Hendrik van de Hulst

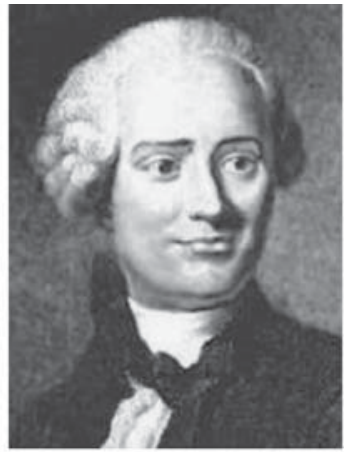

Johann Lambert

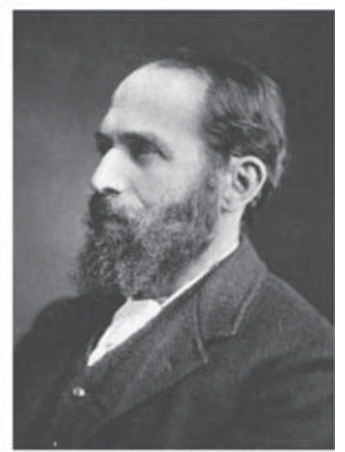

Arthur Schuster

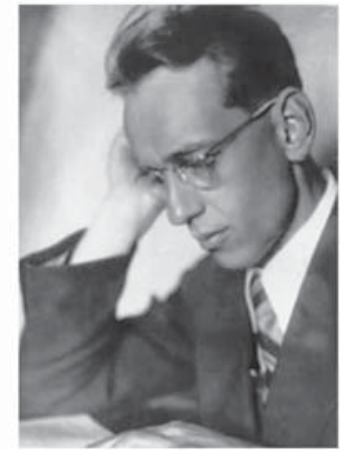

Andrei Gershun

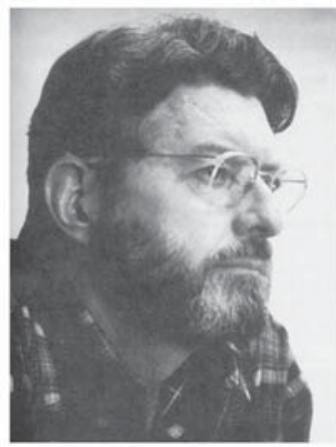

Rudolph Preisendorfer

Fig. 7. Scientists who had contributed to phenomenology and mathematics of directional radiometry and radiative transfer.

The key quantity of the phenomenological photometry and phenomenological RTT is the specific intensity (also called radiance), which is postulated to have primor- dial physical existence and a priori defined properties. The standard definition of the specific intensity was given in 1906 by Max Planck (1858-1947) in his famous Theorie 


\section{T R A I T É \\ D' O P T I Q U E}

GRADATION DE LA LUMIERE:

Ouvrage pofthume de M. BOVGUER, de l'Académie Royale des Sciences, \&C.

Et publís par M. PAbbé DE LA CAILLE, de la même Académic, \&ce.

Pour fervir de Suite aux Mémoires de I Académie Royale des Sciences.

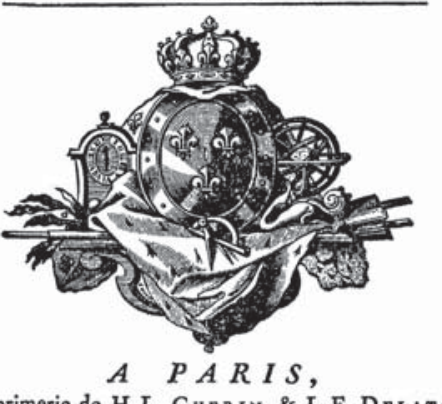

De l'Imprimerie de H.L. GUERIN \& L.F. DenctovR, rue Saint Jacques, à Saint Thomas d'Aquin.

M. D C C. L X.

AVEC APPEOATION ET PEIVILEGE DU ROTO
I. H. LAMBERT

ACADEMIAE SCIENTIARVM ELECTORALIS BOICAE, ET SOCIETATIS PHYSICO-MEDICAE BASILIENSIS MEMBRI, REGIAE SOCIETATI SCIENTIARUM GOETINGENSI COMMERCIO

\section{PHOTOMETRIA SIVE $D E$ \\ MENSVRAET GRADIBVS L V M I NIS, COLORVM ET VMBRAE.}

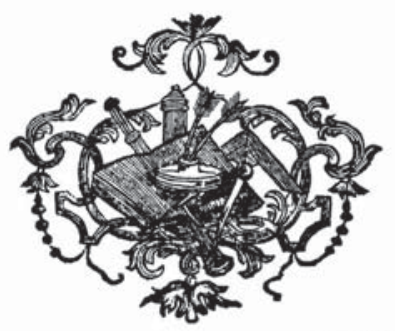

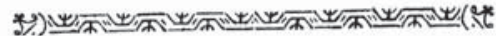

Augustae Vindelicorum, Sumptibus Vidvae Eberhardi Klett Typis Christophort Petri Detleffsen. MDCCLX.

Fig. 8. The title pages of Bouguer's Traité [16] (left) and Lambert's Photometria [17].

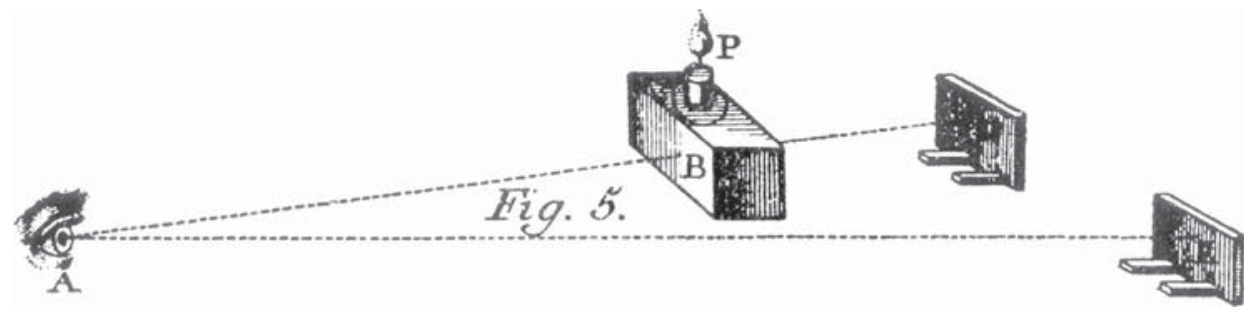

Fig. 9. Experimental setup used by Bouguer to discover the exponential attenuation law.

der Wärmestrahlung [34] (Fig. 11). On page 1 of the English edition of this book [35], one can find the following manifesto:

The state of the radiation at a given instant and at a given point of the medium cannot be represented... by a single vector (that is, a single directed quantity). All heat rays which at a given instant pass through the same point of the medium are perfectly independent of one another, and in order to specify completely the state of the radiation the intensity of radiation must be known in all the directions, infinite in number, which pass through the point in question.

Based on this premise, the monochromatic radiance $\widetilde{I}(\mathbf{r}, \hat{\mathbf{q}})$ is defined by stating that the amount of monochromatic radiant energy $\mathrm{d} E$ transported through an arbitrarily chosen differential element of area $\mathrm{d} S$ in the interior of a medium in directions confined to a differential element of solid angle $\mathrm{d} \Omega_{\hat{\mathbf{q}}}$, centered around the propagation direction $\hat{\mathbf{q}}$, during a differential time interval $\mathrm{d} t$ is given by

$\mathrm{d} E=\widetilde{I}(\mathbf{r}, \hat{\mathbf{q}}) \cos \theta \mathrm{d} S \mathrm{~d} t \mathrm{~d} \Omega_{\hat{\mathbf{q}}}$,

where $\mathbf{r}$ is the position vector of the differential surface element and $\theta$ is the angle between $\hat{\mathbf{q}}$ and the normal $\hat{\mathbf{n}}$ to $\mathrm{d} S$ (Fig. 12a). This definition was eventually adopted in the classical works by E. Arthur Milne [36], Eberhard Hopf [37], and Subramanyan Chandrasekhar [38] as well as in virtually all subsequent monographs and textbooks on the RTT and directional radiometry (see, e.g., Refs. [9,11,39-61]). Although in his treatise Planck specifically considered black-body electromagnetic radiation, his concept of the specific intensity was extended to encompass the scattering of light by cloudy and 
Photometrie der diffusen Zurückwerfung.

473

XI. Die Photometrie der diffusen Zurückwerfung; von E. Lom mel.

(Aus den Sitzungsber. d. math. phys. Classe d. K. Aead. zu Münehen, mitgetheilt voin Hrn. Verf)

In einer früheren Abhandlung ,über Fluorescenz ${ }^{(41}$ ) habe ich in einem: "Ueber die Grundsătze der Photometrie", ũberschriebenen Abschnitt gezeigt, dass in der theoretischen Photometrie nicht, wie bis dahin üblich war, die Flăchenelemente einer leuchtenden Oberfläche, sondern die Volumenelemente des leuchtenden Körpers als lichtstrahlend zu betrachten seien. Demgemăss wurden der theoretischen Behandlung photometrischer Probleme die folgenden drei Sătze zu Grunde gelegt:

I. Die von einem Volumenelement nach einem anderen strahlende Lichtmenge ist dem Quadrate ihrer Entfernung umgekehrt proportional.

II. Die von einem Volumenelement ausstrahlende und auf ein Flächenelement fallende Lichtmenge ist dem Cosinus des Incidenzwinkels proportional.

III. Das von einem Volumenelement ausstrahlende Licht wird auf seinem Wege innerhalb des strahlenden Körpers nach Masssgabe des Absorptionsgesetzes geschwächt.

1) Lommel, Wied. Ann. 10. p. 449 u. 631.1890.

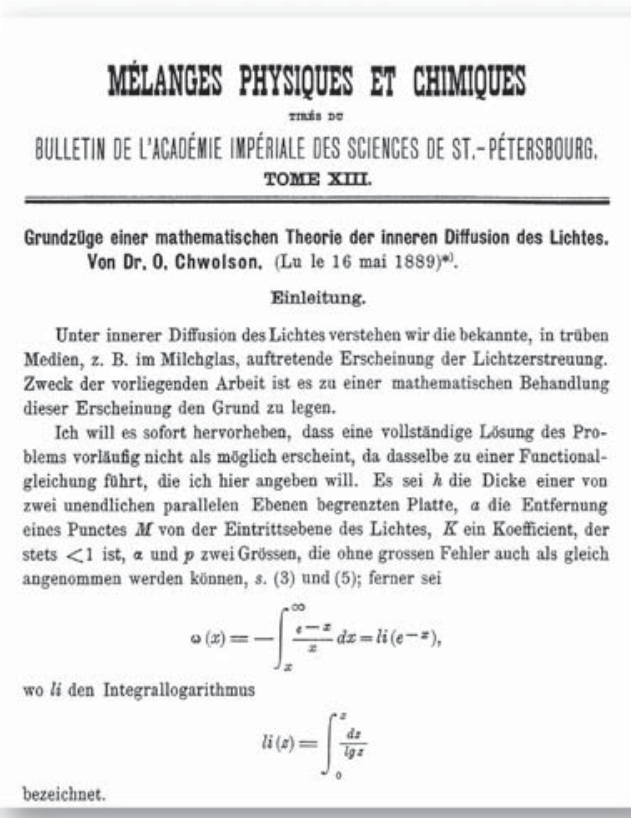

Fig. 10. The first pages of Refs. [29] (left) and [30].

other particulate media, which is the specific subject of this Essay. The heuristic notion of the radiance was eventually supplemented by the traditional belief that it can be directly measured with a suitable optical device such as the so-called Gershun tube sketched in Fig. 13 [21,47,55].

The French physicist and astronomer (as well as the 25th Prime Minister of France) Dominique François Arago (1786-1853) was among the first to criticize phenomenological radiometry for complete ignorance of polarization state of light. This criticism was nominally addressed by replacing the radiance $\widetilde{I}(\mathbf{r}, \hat{\mathbf{q}})$ with the four-element specific intensity column vector $\hat{\mathbf{I}}(\mathbf{r}, \hat{\mathbf{q}})$. In 1924, Richard Gans (1880-1954) considered the transfer of polarized light in a plane-parallel Rayleigh-scattering atmosphere [62], but analyzed only the special case of perpendicularly incident light and considered only the first two components of the specific intensity column vector. The case of arbitrary illumination and arbitrary polarization was addressed in 1950 by Subrahmanyan Chandrasekhar (1910-1995) [38]. In a 1955 paper never translated into English, Georgi Rozenberg (1914-1982) introduced the most general form of the integro-differential vector RTE applicable to sparse scattering media composed of arbitrarily shaped and arbitrarily oriented particles [63]. This publication (see also Ref. [64]) had essentially concluded the conceptual development of the phenomenological RTT. Perhaps the only significant subsequent augmentation was the inclusion of the thermal emission vector in the Rozenberg's vector RTE by Leung Tsang in 1984 [65] (corrected in Ref. [66]).

The work by E. Arthur Milne (1896-1950), Eberhard Hopf (1902-1983), Viktor Ambartsumian (1908-1996), Subramanyan Chandrasekhar, Viktor Sobolev (1915-1999), and Hendrik van de Hulst (1918-2000), among others, had served to establish the phenomenological RTT as a branch of mathematical physics (see Refs. [67-75] and references therein). Most recently, the phenomenological RTT has been incorporated into the equally phenomenological discipline of computer graphics studying techniques to digitally synthesize and manipulate images (see, e.g., Refs. [76-78] and references therein).

\section{What is fundamentally wrong with the phenomenological approach to directional radiometry and RTT?}

\subsection{Polydirectional flow of radiant energy}

In the introduction to the English translation of Gershun's treatise [21], Moon and Timoshenko wrote in 1939:

Theoretical photometry constitutes a case of "arrested development", and has remained basically unchanged since 1760 while the rest of physics has swept triumphantly ahead. In recent years, however, the increasing needs of modern lighting technique have made the absurdly antiquated concepts of traditional photometric theory more and more untenable.

The Gershun's treatise was then presented as part of "a vigorous attempt to bring the theory of light calculation into conformity with the spirit of physics." However, some 70 years after Maxwell's Dynamical Theory [79], Gershun's way of conforming with Maxwell's electromagnetics was, in fact, to ignore it. Instead, the centerpiece of his treatise is the so-called "light field", i.e., "a part of space studied from the standpoint of transmission of radiant energy 


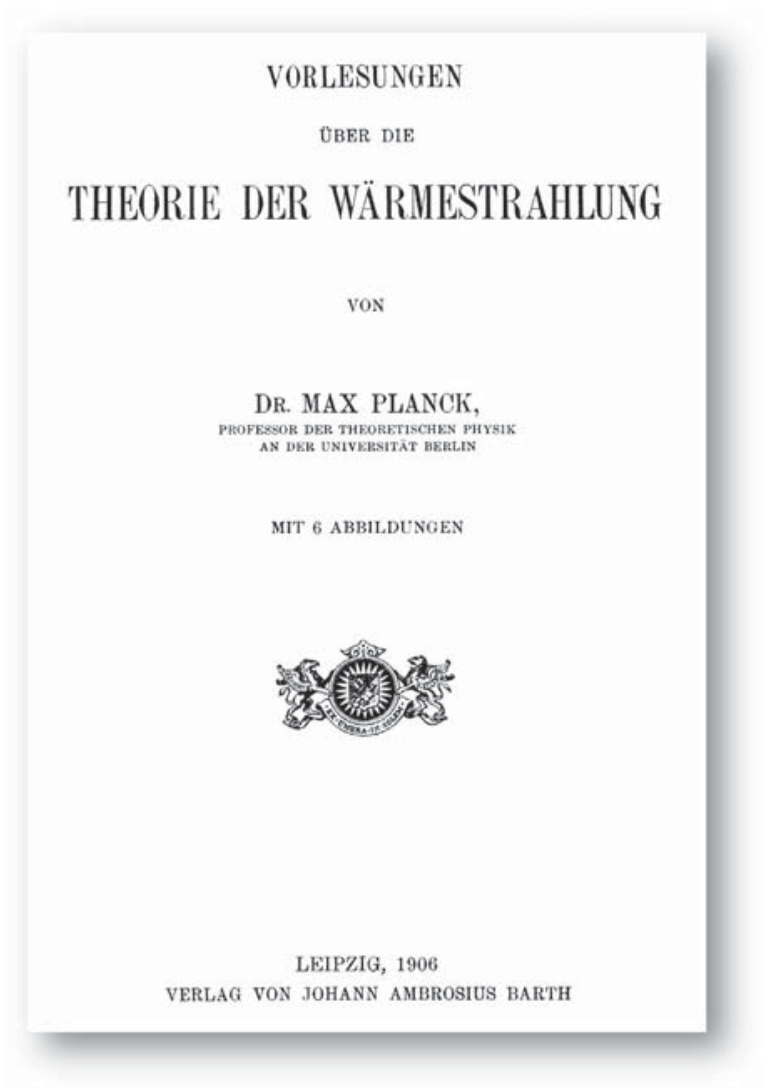

Fig. 11. The title page of Planck's Theorie der Wärmestrahlung [34].

within that space." To justify this approach, Gershun wrote in the introduction to his book:

A reader-physicist would naturally ask why the author distinguishes the light field from the electromagnetic field well studied in physics. It is true that the light field is caused by the electromagnetic field, but qualitatively it is quite different. ${ }^{7}$

After this programmatic statement, electromagnetics is hardly mentioned in the rest of Ref. [21]. This manifesto, according to which the radiance field is somehow qualitatively different from the electromagnetic field and thus must be studied using different physical principles, has been implicit in the majority of "phenomenological" publications on directional radiometry and radiative transfer.

Whether spelled out explicitly or not, the key premise of phenomenological photometry as well as of the phenomenological RTT is that matter interacts with the energy of the electromagnetic field rather than with the electromagnetic field itself. This profoundly false assumption explains the deceitful simplicity of the phenomenological concepts as well as their ultimate failure. Indeed, the very outset of both phenomenological disciplines is the postulation of the existence of the radiance as the primordial

\footnotetext{
${ }^{7}$ This translation is somewhat different from that by Moon and Timoshenko and, in my opinion, is more accurate.
}

physical quantity describing the "instantaneous directional distribution of the radiant energy flow" at a point in space. This is followed by a "derivation" of the scalar RTE on the basis of "simple energy conservation considerations" and the postulation that it is the electromagnetic energy rather than the electromagnetic field that gets scattered by particles and surfaces.

However, it is imperative to recognize that according to classical electromagnetics, the field-matter interaction is controlled by the electric and magnetic field vectors rather than by the energy of the electromagnetic field $[80,81]$. Similarly, the canonical formulation of classical electrodynamics is based on expressing the field-matter interaction term in the Lagrangian and Hamiltonian densities in terms of the electromagnetic 4-vector potential, which, in turn, is again related to the electric and magnetic fields. The canonical formulation of quantum electrodynamics (QED) is obtained by promoting the electromagnetic 4-vector potential and the current density of matter to field operators that satisfy specific commutation relations [82-88]. This implies that any attempt to bypass the explicit solution of the Maxwell equations or an explicit QED computation in a first-principle development of the RTT and thus build the RTT on the notion of radiant energy rather than on the notion of the electromagnetic field (see, e.g., Ref. [89]) is fundamentally flawed and is doomed from the very outset.

Furthermore, the very notion of polydirectional propagation of electromagnetic energy at a point in space, as allegedly described by the radiance, contradicts basic laws of classical electromagnetics and does not follow from QED. Indeed, the quantity characterizing instantaneous electromagnetic energy transport in classical electromagnetics is the Poynting vector $\mathbf{S}$ given by the vector product of the real-valued electric, E, and magnetic, $\mathbf{H}$, field vectors:

$\mathbf{S}(\mathbf{r}, t)=\mathbf{E}(\mathbf{r}, t) \times \mathbf{H}(\mathbf{r}, t)$,

where $\mathbf{r}$ is the position vector and $t$ is time [80,81]. However, by virtue of being a direct corollary of the Maxwell equations, the famous Poynting theorem involves the integral of the Poynting vector over a closed surface. As such, it quantifies the energy budget of a finite volume element rather than the local flow of electromagnetic energy at a point in space. Back in 1916, the Dutch physicist Hendrik Antoon Lorentz (1853-1928) already warned against too literal an interpretation of the Poynting vector as describing a current of electromagnetic energy by noting that

in general it will not be possible to trace the paths of parts or elements of energy in the same sense in which we can follow in their course the ultimate particles of which matter is made up. ...It might even be questioned whether, in electromagnetic phenomena, the transfer of energy really takes place in the way indicated by Poynting's law

(see pages 25-26 of The Theory of Electrons [90]). Even if the Poynting vector could be claimed to characterize the local current of electromagnetic energy, this vector is inherently monodirectional at any moment in time and remains monodirectional upon averaging over any time interval. Therefore, there is no reason whatsoever to 
a

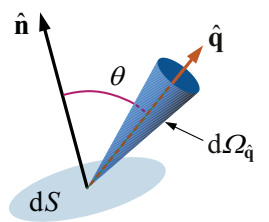

b

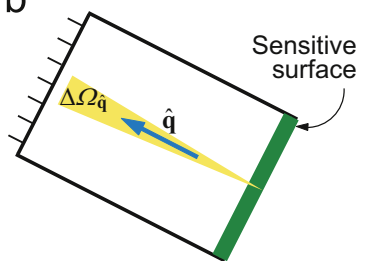

d

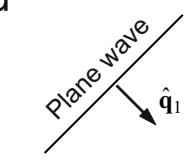

C
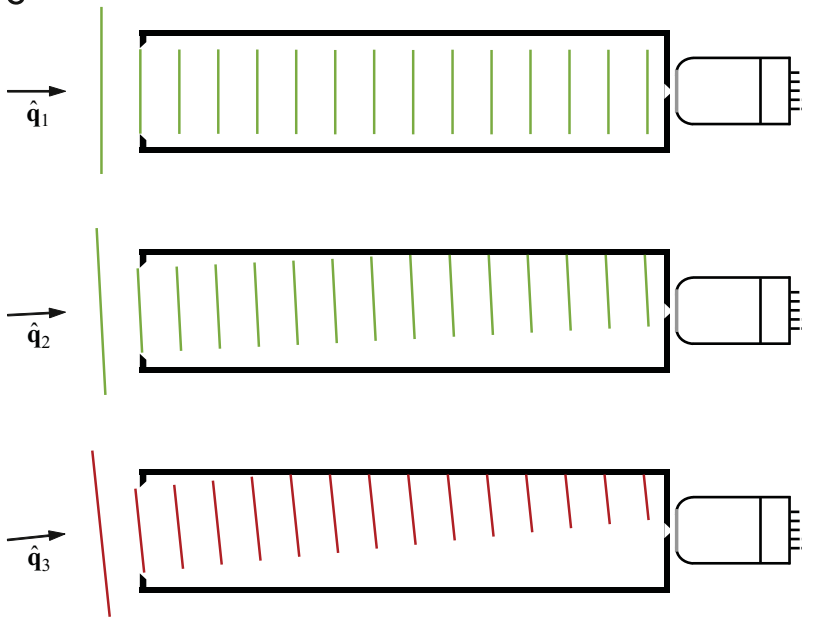

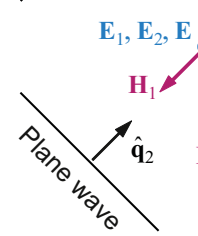

e

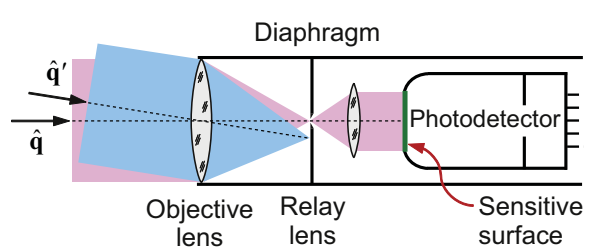

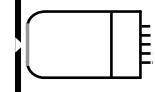

f

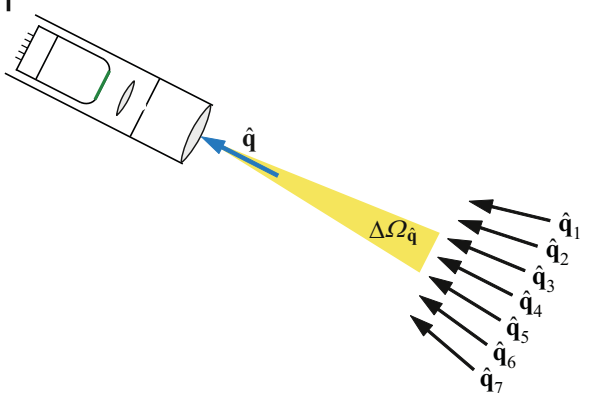

Fig. 12. (a) Definition of the phenomenological specific intensity. (b) Hypothetical radiance function meter accumulating local instantaneous Poynting vectors with directions falling within the acceptance solid angle $\Delta \Omega_{\hat{\mathbf{q}}}$. (c) The Gershun tube is a wavefront filter. (d) The Gershun tube does not respond to the Poynting vector directed along the optical axis of the instrument. (e) Typical optical scheme of a lens-based WCR. (f) Response of a lens-based WCR to a superposition of plane electromagnetic waves.

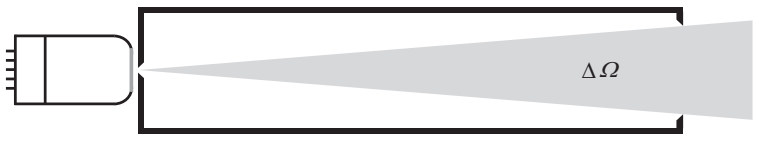

Fig. 13. The Gershun tube is a simple optical device consisting of a long tube with entrance and exit apertures followed by a photodetector. The internal walls of the Gershun tube are assumed to be perfectly absorbing.

postulate that there exists a primordial physical quantity, viz., the radiance, that characterizes the polydirectional local flow of electromagnetic energy.

\subsection{Photonic confusion}

The often uncontrollable use of the word "photon" taken out of its proper QED framework is another manifestation of the implicit desire to bypass the complexities of dealing with the electromagnetic field. Indeed, a popular way to justify the primordial existence of the polydirectional radiance is to claim that the light field is in fact a "gas" of localized point-like particles of light called photons. Usually this is done with reference to Albert Einstein (1879-1955) who attempted to model the photoelectric effect heuristically by resurrecting the idea of light corpuscles advocated by Sir Isaac Newton (1643-1727). Specifically, Einstein suggested in 1905 that

the energy of a light ray spreading out from a point source is not continuously distributed over an increasing space but consists of a finite number of energy quanta which are localized at points in space, which move without dividing, and which can only be produced and absorbed as complete units 
(see Ref. [91]). Since such localized point-like photons must exist in vast numbers, one can imagine that at any given moment many photons can fly by a point in space in different directions, thereby representing the sought polydirectional localized transport of radiant energy [92-96].

The photons are not allowed to collide with each other, but they are allowed to collide with macroscopic particles such as cloud droplets. In the words of Kirk [46], "the photons follow a zig-zag path as they ricochet from one scattering particle to the next." These "ricochets" are random and are claimed (again, on the basis of "simple physical considerations") to lead to a Boltzmann type kinetic equation, viz., the scalar RTE. Unfortunately, it has never been explained why the kernel of this equation, which by design describes the "ricochet" of a photon from a macroscopic particle, is always calculated by solving the classical macroscopic Maxwell equations (MMEs) which involve neither photons nor "photon-particle collisions." Apparently, it is believed that upon approaching a droplet the photon becomes an electromagnetic wave. This allows the photon to "ricochet" from the droplet according to the MMEs (e.g., according to the formulas of the Lorentz - Mie theory) and become an outgoing spherical wave. Eventually, the outgoing spherical wave switches back to being a bunch of localized point-like photons. Needless to say, such mysterious transformations of photons into waves and then back into photons can only happen in the confused human mind rather than in nature. The only outcome of such verbal speculations can be - and has been - the notorious "photonic confusion".

The lasting misinterpretation of the actual QED photons as localized particles of light has been kept flourishing by scores of incompetent popularizers of science, contributors of amateur Wikipedia articles, and authors of many school and college textbooks and even professional monographs. A typical example is the textbook by Taylor et al. [97]. On p. 139, one can find the following misleading statement: "Today all physicists accept that the photoelectric effect, the Compton effect, and numerous other experiments demonstrate beyond doubt the particle nature of light." This statement ignores the well-established fact that the alleged particle behavior of light in phenomena such as the photoelectric effect can be explained quantitatively in terms of the semi-classical approach wherein the electromagnetic field is not quantized and is described by the classical microscopic Maxwell equations [98-100]. Furthermore, based on the advanced QED theory of the photoelectric effect, Kimble and Mandel [101] concluded that photodetectors do not count photons in any precise sense. For some reason these facts are virtually never mentioned in school and college textbooks. Another profoundly wrong statement can be found on p. 125 of Ref. [97]: "It was found that an electromagnetic wave consists of tiny localized bundles of energy. These bundles, or quanta of light, have come to be called photons". Similarly, McCluney [47] speaks of "photon flux", "photon intensity", "photon radiance", and "photon irradiance", while Petty [102] characterizes electromagnetic radiation as a "shower of particles". In Ref. [103] "a beam of radiation is looked upon as a stream of particles called photons", while photons are looked upon "as discrete blobs of energy without phases".
However, the obsolete heuristic ${ }^{8}$ nature of Einstein's localized light quanta [104-106] becomes patently obvious upon opening an advanced textbook on the QED or quantum optics (e.g., Refs. [107-110]). Indeed, although the term "photon" is ubiquitous in those disciplines, it is well recognized that there is no position operator for an actual QED photon and that it is impossible to define a photon wave function in the coordinate representation, which precludes photon localizability in space (e.g., Section 2.2 of Ref. [83]). The real QED photons are quantum excitations of the normal modes of the electromagnetic field and as such are associated with electromagnetic plane waves of definite wave vector and definite polarization but infinite lateral extent. These factors imply that QED photons are not localized point-like particles of light and as such cannot be used to justify the notion of polydirectional local flow of radiant energy allegedly described by the specific intensity [111].

Some 85 years since the development of QED by Paul Adrien Maurice Dirac, Ernst Pascual Jordan, and Werner Karl Heisenberg [112-115], the superficial use of the word "photon" is still frequently accompanied by the mention of the alleged "wave-particle duality" of light. It is therefore instructive to quote from the famous 1995 Anti-Photon by Willis Lamb Jr. [106], where he notes that talking

about the wave - particle duality in discussion of quantum mechanics ... may be necessary for those who are unwilling or unable to acquire an understanding of the theory. However, this concept is even more pointlessly introduced in discussions of problems in the quantum theory of radiation.

He concludes the Anti-Photon by stating that

It is high time to give up the use of the word "photon", and of a bad concept which will shortly be a century old. Radiation does not consist of particles, and the classical, i.e., non-quantum, limit of the quantum theory of radiation is described by Maxwell's equations for the electromagnetic field, which do not involve particles. Talking about radiation in terms of particles is like using such ubiquitous phrases as "You know" or "I mean" which are very much to be heard in some cultures. $^{9}$

In 1989, Kidd et al. [105] suggested that elementary texts would benefit from dropping the corpuscular photon (except, perhaps, as a historical topic) and switching to the semi-classical treatment as the first approximation to the modern QED approach. In 1995, Lamb Jr. [106] wrote that "the sooner an appropriate reformulation of our educational processes can be made, the better." However, it

\footnotetext{
${ }^{8}$ Einstein himself recognized the phenomenological character of his 1905 paper by giving it the title "Concerning an heuristic point of view toward the emission and transformation of light" [91].

9 I must admit my own "guilt" of misusing the word "photon" in several early publications. A typical example is the physically meaningless expression "weak localization of photons" used in lieu of the correct (but less catchy) expression "weak localization of electromagnetic waves".
} 
appears that imaginary point-like photons continue to proliferate, while the inability to explain the nature of the actual QED photon is still disguised by references to the mysterious "wave - particle duality" of light.

\subsection{Preisendorfer's radiance function}

The need to establish a conceptual link between the "island" of the phenomenological RTT and the "mainland of electromagnetic theory" was eloquently formulated in 1965 by Rudolph Preisendorfer (see Chapter XIV of Ref. [28]). He admitted that the heuristic polydirectional radiance cannot be defined as existing at a specific moment $t$ but rather must be the result of averaging over a sufficiently long time interval. He observed that in a turbid medium, the constituent particles are in constant motion and can also change their sizes, shapes, and orientations, thereby rendering the direction and magnitude of the Poynting vector $\mathbf{S}(\mathbf{r}, t)$ at an observation point $\mathbf{r}$ random functions of time. At certain moments the direction of the instantaneous Poynting vector can fall within the differential solid angle $\mathrm{d} \Omega_{\hat{\mathbf{q}}}$ in Fig. 12a. Therefore, Preisendorfer suggested that an appropriate definition of the specific intensity could be the time-averaged length of the Poynting vectors at $\mathbf{r}$ with directions falling within $\mathrm{d} \Omega_{\hat{\mathbf{q}}}$. More specifically, Preisendorfer's radiance function is defined as follows (see Fig. 12a):

$$
\begin{aligned}
\widetilde{N}(\mathbf{r}, \hat{\mathbf{q}})= & \frac{1}{\cos \theta} \lim _{\Delta S \rightarrow 0} \frac{1}{\Delta S} \lim _{\Delta \Omega_{\hat{\mathbf{q}}} \rightarrow 0} \frac{1}{\Delta \Omega_{\hat{\mathbf{q}}}} \lim _{T \rightarrow \infty} \frac{1}{T} \int_{\Delta S} \mathrm{~d}^{2} \mathbf{r}^{\prime} \\
& \times \int_{t-(T / 2)}^{t+(T / 2)} \mathrm{d} t^{\prime}\left|\mathbf{S}\left(\mathbf{r}^{\prime}, t^{\prime}\right)\right| \chi\left[\Delta \Omega_{\hat{\mathbf{q}}}, \hat{\mathbf{s}}\left(\mathbf{r}^{\prime}, t^{\prime}\right)\right],
\end{aligned}
$$

where $\hat{\mathbf{s}}\left(\mathbf{r}, t^{\prime}\right)=\mathbf{S}\left(\mathbf{r}, t^{\prime}\right) /\left|\mathbf{S}\left(\mathbf{r}, t^{\prime}\right)\right|$ is the unit vector in the direction of $\mathbf{S}\left(\mathbf{r}^{\prime}, t^{\prime}\right)$ and

$\chi\left[\Delta \Omega_{\hat{\mathbf{q}}}, \hat{\mathbf{s}}\left(\mathbf{r}^{\prime}, t^{\prime}\right)\right]= \begin{cases}1 & \text { if } \hat{\mathbf{s}}\left(\mathbf{r}^{\prime}, t^{\prime}\right) \in \Delta \Omega_{\hat{\mathbf{q}}} \\ 0 & \text { otherwise }\end{cases}$

is the angular step function. Essentially the same definition of the radiance can be found in Section 7.9 of Ref. [12]. Preisendorfer then attempted to use the Maxwell equations to demonstrate that his radiance function $\widetilde{N}(\mathbf{r}, \hat{\mathbf{q}})$ satisfies the scalar RTE.

However, Preisendorfer's attempt to bridge the gap between the phenomenological RTT and Maxwell's electromagnetics failed for several reasons. First of all, we have already mentioned that there is no fundamental reason to believe that the Poynting vector specifies the direction and magnitude of the instantaneous local flow of electromagnetic energy. Secondly, the derivation of the RTE in Ref. [28] turned out to be incorrect owing to the wrong underlying assumption that the instantaneous electric and magnetic field vectors at any point inside a turbid medium are always mutually orthogonal. Thirdly, it can be demonstrated that the radiance function $\widetilde{N}(\mathbf{r}, \hat{\mathbf{q}})$ does not satisfy the RTE [116]. Fourthly, even if $\widetilde{N}(\mathbf{r}, \hat{\mathbf{q}})$ were to satisfy the RTE, this quantity would be useless because it cannot be measured at a point inside a volume of turbid medium.
To illustrate the last statement, let us assume that there exists a hypothetical instrument that reacts to the magnitude of the instantaneous local Poynting vector only if the direction of $\mathbf{S}\left(\mathbf{r}^{\prime}, t\right)$ falls within a narrow acceptance solid angle $\Delta \Omega_{\hat{\mathbf{q}}}$, where $\mathbf{r}^{\prime}$ is a point on the sensitive surface $S$ and the unit vector $\hat{\mathbf{q}}$ specifies the orientation of the optical axis of the instrument (Fig. 12b). Let us also assume that this hypothetical instrument is placed inside a random cloud consisting of $N$ particles (Fig. 14). It is quite obvious that averaging the reading of such an instrument over a sufficiently long period of time would essentially yield the Preisendorfer's radiance function $\widetilde{N}(\mathbf{r}, \hat{\mathbf{q}})$ provided that the presence of the instrument does not affect the instantaneous Poynting vector $\mathbf{S}\left(\mathbf{r}^{\prime}, t\right)$ for any $\mathbf{r}^{\prime} \in S$, where $\mathbf{r}$ is the position vector of the central point of the sensitive surface.

However, it is easily seen that no matter how small the instrument is relative to the cloud, its very presence serves to not just affect, but completely destroy the quantity that it is supposed to react to [117]. Indeed, let us assume for simplicity that the cloud particles are separated widely enough to satisfy the conditions of applicability of the farfield Foldy equations (Section 4.5). Then the total instantaneous electric and magnetic fields at $\mathbf{r}^{\prime}$ in the absence of the detector are superpositions of the respective incident and $N$ partial scattered fields:

$\mathbf{E}\left(\mathbf{r}^{\prime}, t\right)=\mathbf{E}^{\mathrm{inc}}\left(\mathbf{r}^{\prime}, t\right)+\sum_{i=1}^{N} \mathbf{E}_{i}^{\text {sca }}\left(\mathbf{r}^{\prime}, t\right)$,

$\mathbf{H}\left(\mathbf{r}^{\prime}, t\right)=\mathbf{H}^{\mathrm{inc}}\left(\mathbf{r}^{\prime}, t\right)+\sum_{i=1}^{N} \mathbf{H}_{i}^{\mathrm{sca}}\left(\mathbf{r}^{\prime}, t\right)$,

where $\mathbf{E}_{i}^{\text {sca }}\left(\mathbf{r}^{\prime}, t\right)$ and $\mathbf{H}_{i}^{\text {sca }}\left(\mathbf{r}^{\prime}, t\right)$ describe an outgoing spherical wavelet centered at the origin of particle $i$. By definition, the corresponding local instantaneous Poynting vector is given by the vector product $\mathbf{S}\left(\mathbf{r}^{\prime}, t\right)=\mathbf{E}\left(\mathbf{r}^{\prime}, t\right) \times \mathbf{H}\left(\mathbf{r}^{\prime}, t\right)$. The major side effect of the presence of the hypothetical detector is to block the spherical wavelets generated by the $N^{\prime}$ particles located to the left of the plane through the sensitive surface shown schematically by the dashed line in Fig. 14. The resulting "truncated" electric and magnetic fields at $\mathbf{r}^{\prime}$ are now given by

$\mathbf{E}^{\prime}\left(\mathbf{r}^{\prime}, t\right)=\sum_{i=1}^{N-N^{\prime}} \mathbf{E}_{i}^{\mathrm{sca}}\left(\mathbf{r}^{\prime}, t\right)$

$\mathbf{H}^{\prime}\left(\mathbf{r}^{\prime}, t\right)=\sum_{i=1}^{N-N^{\prime}} \mathbf{H}_{i}^{\mathrm{sca}}\left(\mathbf{r}^{\prime}, t\right)$,

where the sums include only the contributions from the $N-N^{\prime}$ "unblocked" particles, and we assume for simplicity that the instrument blocks the incident plane wave as well. It is patently obvious that the corresponding "truncated" Poynting vector is not the same as the original Poynting vector:

$\mathbf{S}^{\prime}\left(\mathbf{r}^{\prime}, t\right)=\mathbf{E}^{\prime}\left(\mathbf{r}^{\prime}, t\right) \times \mathbf{H}^{\prime}\left(\mathbf{r}^{\prime}, t\right) \neq \mathbf{S}\left(\mathbf{r}^{\prime}, t\right)$.

Irrespective of its potential practical utility, the directional detector of electromagnetic energy flow sketched in Fig. 12b has never been built, and it remains unknown whether it can be designed in principle. Obviously, appraising the very feasibility of building a detector with directional sensitivity to the local instantaneous Poynting vector requires an advanced QED analysis of light-matter 


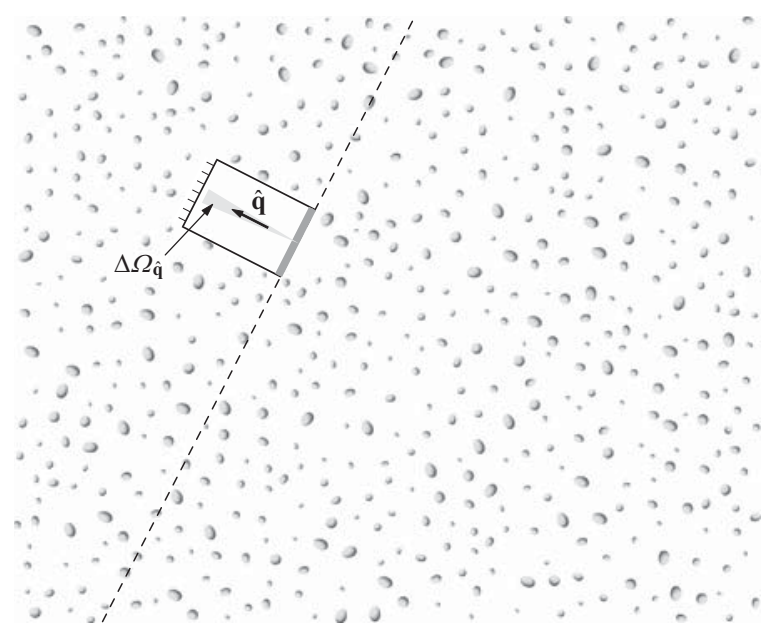

Fig. 14. The hypothetical radiance function meter is placed inside a cloud of randomly positioned and randomly moving particles.

interaction. It is not inconceivable that there exists a Heisenberg uncertainty relation fundamentally prohibiting such a measurement.

\subsection{Directional radiometers}

Another profound misconception of phenomenological radiometry is the belief that instruments like the Gershun tube (Fig. 13) measure the directional flow of electromagnetic energy, i.e., filter out the energy propagating within the acceptance solid angle

$\Delta \Omega=\frac{\pi D_{\mathrm{en}}^{2}}{4 L^{2}}$,

where $D_{\mathrm{en}}$ is the diameter of the entrance aperture and $L$ is the length of the tube (the diameter of the exit aperture $D_{\text {ex }}$ is assumed to be much smaller than $D_{\text {en }}$ ). Instead, an elementary physical-optics analysis shows that the Gershun tube filters out plane or near plane electromagnetic wavefronts with propagation directions $\hat{\mathbf{q}} \in \Delta \Omega$. Let us consider, for example, the incident field in the form of a superposition of three plane waves propagating in the directions $\hat{\mathbf{q}}_{1}, \hat{\mathbf{q}}_{2}$, and $\hat{\mathbf{q}}_{3}$, respectively, as shown separately in the three panels of Fig. 12c. The diffraction of the incident field on the circular entrance aperture of the Gershun tube is linear in the field: the total field inside the tube is the superposition of the fields resulting from the diffraction of the three individual components of the incident field. The standard theory of Fresenel diffraction on a circular aperture (e.g., Ref. [118]) implies that if the corresponding Fresnel number $N_{\mathrm{F}}=D_{\mathrm{en}}^{2} / \lambda L \rightarrow \infty$, where $\lambda$ is the wavelength, then the circular part of an incident plane wave cut out by the entrance aperture propagates inside the tube essentially unchanged. It is then clear that the truncated wavefronts propagating in the directions $\hat{\mathbf{q}}_{1}$ and $\hat{\mathbf{q}}_{2}$ will reach the exit aperture and contribute to the reading of the photodetector, whereas the entirety of the truncated wavefront propagating in the direction $\hat{\mathbf{q}}_{3}$ will be absorbed by the black walls of the Gershun tube and will not contribute to the photoelectric signal.
This analysis implies that the Gershun tube does not necessarily react to the local Poynting vector at a point in the entrance aperture even if this vector is directed along the optical axis of the instrument. To demonstrate this, let us consider the electromagnetic field formed by a superposition of two plane electromagnetic waves propagating in directions $\hat{\mathbf{q}}_{1}$ and $\hat{\mathbf{q}}_{2}$ such that both form a $45^{\circ}$ angle with the optical axis of the instrument (Fig. 12d). The waves are linearly polarized, with their electric vectors $\mathbf{E}_{1}$ and $\mathbf{E}_{2}$ oscillating perpendicularly to the paper, and fully coherent in that at any moment in time $\mathbf{E}_{1}=\mathbf{E}_{2}$ at the central point of the entrance aperture. Let the local instantaneous magnetic vectors of the waves be $\mathbf{H}_{1}$ and $\mathbf{H}_{2}$, respectively, as shown by the magenta arrows, while the corresponding instantaneous electric vectors $\mathbf{E}_{1}=\mathbf{E}_{2}$ are directed towards the reader. The cumulative local instantaneous field is given by the vectors $\mathbf{E}=2 \mathbf{E}_{1}$ and $\mathbf{H}=\mathbf{H}_{1}+\mathbf{H}_{2}$, the former again being directed towards the reader. One can see that the resulting local instantaneous Poynting vector $\mathbf{S}=\mathbf{E} \times \mathbf{H}$, shown by the green arrow, is directed along the optical axis of the Gershun tube. Moreover, it is easily verified that the Poynting vector at the central point is always directed along the optical axis of the instrument. Yet the reading of the end photodetector is identically equal to zero since neither truncated wavefront can reach the exit aperture.

Thus, by its very design, the Gershun tube is a wavefront filter with an acceptance solid angle given by Eq. (10). If this angle is sufficiently small then the Gershun tube can be said to be a well-collimated radiometer (WCR) in that it filters out only those plane or near-plane wavefronts that propagate in essentially the same direction given by the optical axis of the instrument.

In fact, the Gershun tube is a very inefficient WCR since its energy collection efficiency is defined only by the area of the exit aperture $\pi D_{\mathrm{ex}}^{2} / 4$. This area can be increased by increasing $D_{\text {ex }}$, but then the acceptance solid angle $\Delta \Omega$ also increases and becomes partially vignetted. Most WCRs in use today are based on a different optical design, as illustrated schematically in Fig. 12e. Now the main functional elements are the objective and relay lenses, the diaphragm, and the end photoelectric detector. Let us consider the response of such a lens-based instrument to the electromagnetic field formed by superposing two plane waves propagating in directions $\hat{\mathbf{q}}$ and $\hat{\mathbf{q}}^{\prime}$, respectively. The objective lens acts as a linear optical transformer in that its effect on the total field is a superposition of its effects on each plane-wave component. Specifically, the well-known paraxial approximation (see, e.g., Section 5.1 of Ref. [119]) implies that in the near zone of the objective lens either plane wavefront is transformed into a converging spherical wavefront with its respective focal point located in the plane of the diaphragm. However, the ultimate fates of the two spherical wavefronts in Fig. 12e are different. The pink spherical wavefront passes freely through the pinhole, is converted back into a plane wavefront, and is relayed onto the sensitive surface of the photodetector, thereby contributing to the cumulative reading of the WCR. The blue spherical wavefront gets annihilated by the diaphragm and does not contribute to the photoelectric signal. 
Thus the combination \{objective lens, diaphragm\} serves to select only plane (or near-plane) wavefronts propagating in directions very close to the optical axis of the instrument and falling within its small acceptance solid angle

$\Delta \Omega=\frac{\pi d^{2}}{4 f^{2}}$

where $d$ is the diameter of the pinhole and $f$ is the focal length of the objective lens. The key advantage of this design is that the energy-collection efficiency, defined by the diameter of the objective lens, and the acceptance solid angle are now independent of each other, so that the former can be increased without degrading the latter.

Thus, contrary to a widespread belief, a WCR cannot be said in general to measure the directional distribution of the electromagnetic energy flow at an observation point. It is therefore imperative to formulate precisely what a WCR does in actuality. Let us assume that the lens-based WCR shown in Fig. 12e is exposed to an electromagnetic field in the form of a superposition of several plane wavefronts, as depicted schematically in Fig. 12f. According to the above discussion, the instrument does the following:

- selects only the wavefronts with propagation directions falling within its small acceptance solid angle $\Delta \Omega_{\hat{\mathbf{q}}}$ (i.e., the wavefronts propagating in the directions $\hat{\mathbf{q}}_{3}, \hat{\mathbf{q}}_{4}$, and $\hat{\mathbf{q}}_{5}$, but not in the directions $\hat{\mathbf{q}}_{1}, \hat{\mathbf{q}}_{2}, \hat{\mathbf{q}}_{6}$, and $\hat{\mathbf{q}}_{7}$ );

- sums up the respective instantaneous electric and magnetic field vectors: $\mathbf{E}^{\prime}=\mathbf{E}_{3}+\mathbf{E}_{4}+\mathbf{E}_{5}$ and $\mathbf{H}^{\prime}=\mathbf{H}_{3}+$ $\mathbf{H}_{4}+\mathbf{H}_{5}$; and finally

- integrates the modulus of the vector product $\mathbf{E}^{\prime} \times \mathbf{H}^{\prime}$ (which, by its very construct, is always directed along or very close to - the optical axis of the WCR) over the objective lens as well as over time.

The Gershun tube does almost the same, except now the vector product $\mathbf{E}^{\prime} \times \mathbf{H}^{\prime}$ is integrated over the small exit aperture.

The reader should find it quite instructive to recognize that despite seemingly being quite different, the one natural and four manmade devices shown in Fig. 15 perform the same physical operation of filtering out electromagnetic wavefronts rather than electromagnetic energy currents. Some of these devices can be equipped with twodimensional pixelated detectors such as a CCD or retina, in which case each pixel has the same functionality as the diaphragm in Fig. 12e besides being an individual photodetector. In the final analysis, all these devices are WCRs, perhaps with the added panoramic capability.

It is remarkable that despite the massive practical use of optical instruments such as those shown in Figs. 12e and 13 for many decades, the fundamental physical principle of the corresponding measurements had not been recognized until quite recently [117]. We will see in the following section that once this principle had been clearly stated, it became possible to formulate the discipline of directional radiometry as a branch of physical optics.

\section{Path to physical optics}

The tenuous standing of the centuries-old phenomenological disciplines of directional radiometry and radiative transfer with respect to fundamental physics has been recognized for decades [12,21,28,64,109,111,121]. Over the past 50 years many important studies have been published with the goal of developing the requisite microphysical (i.e., back-traceable to the MMEs) foundation for both disciplines (e.g., Refs. [121-139] and references therein). In most cases the heuristic concept of the local polydirectional radiance has been viewed as worth being preserved despite being poorly defined. This has led to attempts to identify a quadratic form in the electric (or electric and magnetic) field vectors that would satisfy the RTE and thereby might be considered a legitimate microphysical proxy for the phenomenological specific intensity. However, those studies have not clarified the issue of the requisite non-negativity and physical measurability of such proxies as well as have not established their relevance to the actual electromagnetic energy transport in turbid media. Furthermore, in many cases the scalar wave equation has been used in lieu of the Maxwell equations. Some studies have been based on artificial mathematical models of a random electromagnetic field wherein certain statistical characteristics of the randomness were prescribed a priori rather than derived from direct solutions of the Maxwell equations for statistically random scattering media. It was thus assumed that the regime of radiative transfer must be attributed to a certain random behavior of the sources of the electromagnetic field rather than to the random behavior of the scattering medium. However, this assumption rules out the case of illumination of a turbid medium by a monochromatic or quasi-monochromatic parallel beam and as such has been shown to be generally incorrect.

As a consequence of various shortcuts, the majority of these publications have achieved only partial success in establishing the microphysical foundation of the RTT and directional radiometry. As recently as in 1995, Leonard Mandel and Emil Wolf remarked (see page 287 of Ref. [109]) that in spite of the long history of radiometry and the theory of radiative energy transfer, their foundations had not been fully clarified.

The final solution of the problem in the case of electromagnetic scattering by a turbid medium has become possible only with the recent realization that one does not need to use the specific intensity as the fundamental point of departure and contemplate it as an actual physical quantity required to possess certain desirable properties as well as satisfy the RTE. Instead of declaring the overarching objective of identifying a microphysical quantity that would allow one to derive the RTE for its own sake, one needs to focus on addressing the following problems that are consistent with the very structure and range of applicability of Maxwell's electromagnetics as well as have actual and straightforward practical importance:

1. How to evaluate theoretically the time-averaged radiation-energy budget of a macroscopic volume of random particulate medium?

2. Given the widespread practical use of WCRs, how to model theoretically the particular measurement afforded by a WCR and thereby clarify its ability to serve as (i) an energy-budget instrument and/or (ii) an integral part of a 
a

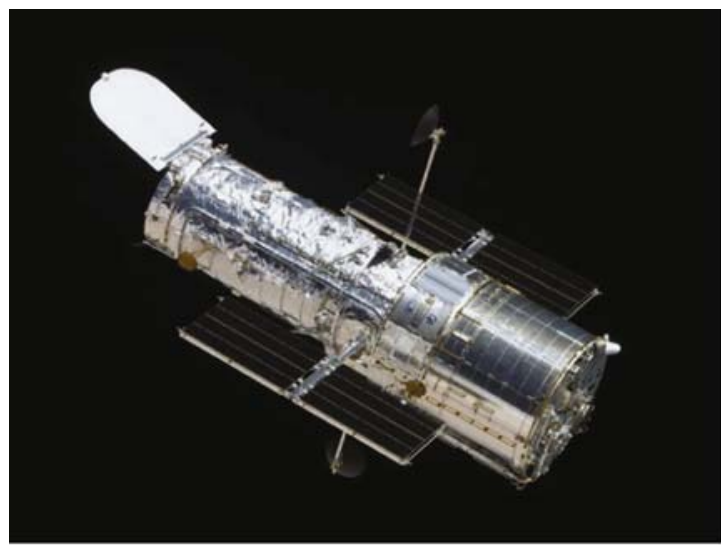

C

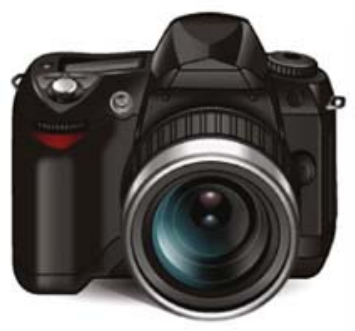

b
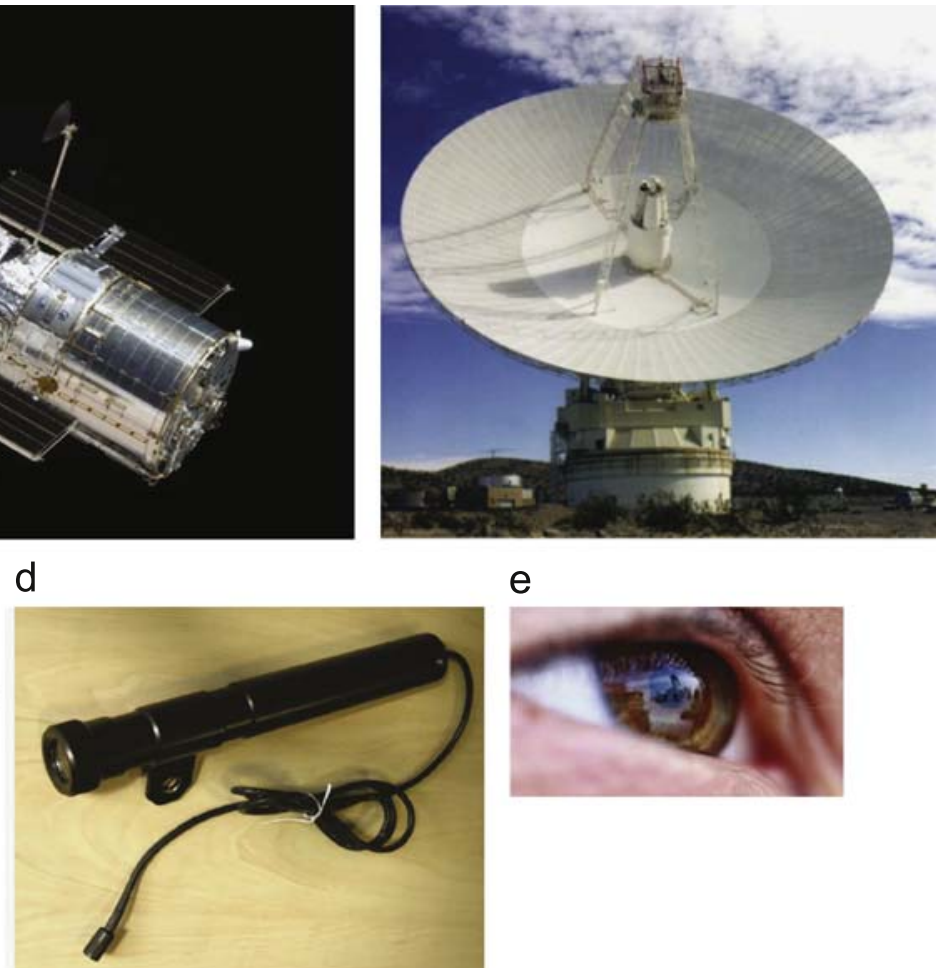

e

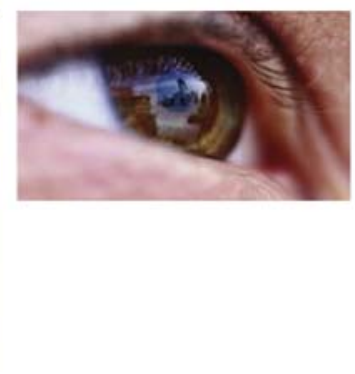

Fig. 15. (a) NASA's Hubble Space Telescope. (b) NASA's 64-m Goldstone radio telescope. (c) Digital photographic camera. (d) Gershun tube [120]. (e) Human eye.

diagnostic technique intended for optical characterization of a particulate medium in a laboratory, in situ, or remotesensing arrangement?

Indeed, it is the solution of these two specific and welldefined problems that one needs in the final analysis, the hypothetical "angular distribution of the local electromagnetic energy flow" being irrelevant and unnecessary in addition to being unphysical. Both problems have been addressed by directly and self-consistently solving the Maxwell equations, as applied to a turbid medium, in Refs. $[116,140]$ (see also Refs. [117,141]).

The process of establishing the microphysical foundation of the disciplines of directional radiometry and radiative transfer has been rather long and convoluted. In what follows, I will try to highlight the major milestones of this endeavor. Certain key developments, such as the discovery of the electromagnetic nature of light, have not been rooted specifically in either discipline and have a much broader significance. Yet they have provided the requisite conceptual framework and as such will also be mentioned.

\subsection{Maxwell's electromagnetics}

Although the pioneering research by Thomas Young (1773-1829), Augustin Jean Fresnel (1788-1827), and Sir George Gabriel Stokes (1819-1903; see Fig. 16) had established the wave nature of light, it was the existence of a specific plane-wave solution of the MMEs that had led
James Clerk Maxwell (1831-1879) to conclude that light consists of electromagnetic waves. Although visible light was the only part of the electromagnetic spectrum known to Maxwell, subsequent research led to the discovery of other parts of the spectrum which now extends from gamma rays to radio waves.

Maxwell's electromagnetics was the first relativistic field theory and constitutes one of the supreme intellectual achievements in the history of humankind. Ludwig Boltzmann was so enchanted by the beauty and might of the Maxwell equations that he quoted from Johann Goethe's Faust: "Was it a God who wrote these signs" (see page iii of Ref. [142]). In his magnificent history of mathematical thought from ancient to modern times, Morris Kline wrote: "The most spectacular triumph of the nineteenth century, with an enormous impact on science and technology, was Maxwell's derivation in 1864 of the laws of electromagnetism" (see page 698 of Ref. [143]).

A meticulous account of the history of classical electromagnetics from the time of Gilbert and Descartes to the relativity theory of Poincaré and Lorentz was given by Sir Edmund Taylor Whittaker [144]. Ref. [145] describes how Maxwell's ideas, summarized in his famous Treatise [146], were picked up, organized, and reworked mathematically by his immediate followers, most notably by Oliver Heaviside (1850-1925) [147]. An important "byproduct" of Heaviside's work on electromagnetics was the creation (independently of $\mathrm{J}$. Willard Gibbs) of vector algebra and vector analysis. Jules Henri Poincaré (1854-1912) 
introduced the overarching principle of relativity [148] and derived the relativity theory as a direct corollary of the Maxwell equations [149,150].

The famous Poynting theorem quantifying the energy budget of a finite volume element was derived by John Henry Poynting (1852-1914) in 1884 [151] and independently by Heaviside in 1885 [152]. Lorentz [90] gave the first derivation of the MMEs from classical microscopic electromagnetics. His work was refined and generalized by de Groot and Suttorp [153] and Robinson [154]. The direct derivation of the MMEs from QED has turned out to be a much more complex problem. It is especially involved if a dielectric medium is lossy, in which case the dielectric needs to be linked to a thermal field reservoir. Recent progress in this direction has been substantial [155-157], but further research is still needed [158].

Classical macroscopic electromagnetics is not the most fundamental physical theory of light-matter interactions. In particular, it ignores the discreteness of matter and operates with continuous sources of fields, which implies that its predictions can fall short in cases where quantum effects are essential. In spite of that, the quantum theory can often be used to determine the "bulk" electromagnetic properties of bodies consisting of very large numbers of atoms [158]. It has been demonstrated that this approach works well when the external electromagnetic field is sufficiently weak and the size of individual particles forming a turbid medium exceeds $\sim 50 \AA$ [159]. Obviously, this result implies a rather wide range of practical applicability of the MMEs.

A clear delineation of the type of field-matter interactions captured by classical macroscopic electromagnetics helps identify specific problems that can be addressed by solving the MMEs. For example, let us consider a cloud of liquid-water droplets illuminated by a parallel quasimonochromatic beam of light (Fig. 17) and suppose that we need to evaluate the energy budget of a macroscopic volume element $\Delta V$ bounded by the closed surface $\Delta S$. According to the Poynting theorem, the net average rate at which electromagnetic energy enters this volume element is given by the surface integral

$\left\langle\left\langle W_{\Delta S}\right\rangle\right\rangle=-\int_{\Delta S} \mathrm{~d}^{2} \mathbf{r}\langle\langle\mathbf{S}(\mathbf{r}, t)\rangle\rangle \cdot \hat{\mathbf{n}}(\mathbf{r}) \geq 0$,

where $\langle\langle\cdots\rangle\rangle$ denotes averaging over a sufficiently long period of time and the unit vector $\hat{\mathbf{n}}(\mathbf{r})$ is directed along the local outward normal to the boundary. The meaning of the Poynting theorem is straightforward if $\left\langle\left\langle W_{\Delta S}\right\rangle\right\rangle=0$, in which case the incoming energy is balanced by the outgoing energy and the particulate matter inside $\Delta V$ is not affected by the electromagnetic radiation. However, if $\left\langle\left\langle W_{\Delta S}\right\rangle\right\rangle>0$ and $\Delta V$ contains no free charges then the Poynting theorem implies that there is a continuous accumulation of electromagnetic energy inside $\Delta V$, which is physically unrealistic. This result exposes an inherent weakness of the MMEs rooted in their inability to describe nonlinear field-matter interactions. A conventional "patch" used to circumvent this issue is the postulate that if $\left\langle\left\langle W_{\Delta S}\right\rangle\right\rangle>0$ then the excess electromagnetic energy is transformed into other forms of energy (e.g., heat) via physical mechanisms not specifically described by classical macroscopic electromagnetics.

Another type of practical problems that can be addressed in the framework of classical macroscopic electromagnetics has to do with the fact that instruments such as WCRs are capable of measuring various manifestations of electromagnetic energy flow. The signal measured by these instruments can carry imbedded information on the physical properties of the scattering medium. To extract this information from the signal, one must (i) have a clear understanding of the physical nature of the measurements and (ii) be able to solve the inverse problem of identifying the physical model of a particulate medium that provides the best fit of theoretical computations of electromagnetic scattering to the measurement data. Of course, an integral part of solving the inverse problem is solving the direct problem, i.e., finding an accurate solution of the MMEs for a given model of particulate medium and a specific type of illumination.

\subsection{Electromagnetic scattering}

To evaluate theoretically the time-averaged radiation energy budget of a macroscopic volume of particulate medium or to model theoretically the time-averaged reading of a WCR, one must be able to address the following two problems:

- determine the instantaneous electric and magnetic fields by solving the MMEs for a fixed multi-particle configuration and

- average the Poynting vector and relevant optical observables over a sufficiently long period of time during which particle positions and microphysical states are allowed to randomly change.

The microphysical state of a particle is characterized by its size, morphology (including the potentially inhomogeneous distribution of the relative refractive index), and orientation.

To solve the first problem, it is usually assumed that the fixed $N$-particle group is imbedded in a homogeneous and nonabsorbing infinite host medium. Furthermore, it is assumed that over time intervals much longer than $2 \pi / \omega$, the time dependence of the electric and magnetic fields everywhere in space is harmonic and described, in the complex-field representation, by the simple complex exponential $\exp (-i \omega t)$, where $\omega$ is the angular frequency and $i=(-1)^{1 / 2}$. In other words, it is assumed that the complex electric and magnetic fields can be factorized as $\widetilde{\mathbf{E}}(\mathbf{r}, t)=\exp (-i \omega t) \widetilde{\mathbf{E}}(\mathbf{r})$ and $\widetilde{\mathbf{H}}(\mathbf{r}, t)=\exp (-i \omega t) \widetilde{\mathbf{H}}(\mathbf{r})$, respectively, while the actual real-valued fields are obtained by taking the real part of the respective complex fields: $\mathbf{E}(\mathbf{r}, t)=\operatorname{Re} \widetilde{\mathbf{E}}(\mathbf{r}, t)$ and $\mathbf{H}(\mathbf{r}, t)=\operatorname{Re} \widetilde{\mathbf{H}}(\mathbf{r}, t)$.

The frequency-domain monochromatic Maxwell curl equations for the time-independent electric and magnetic field amplitudes $\widetilde{\mathbf{E}}(\mathbf{r})$ and $\widetilde{\mathbf{H}}(\mathbf{r})$ are as follows:

$\left.\begin{array}{l}\nabla \times \widetilde{\mathbf{E}}(\mathbf{r})=i \omega \mu_{0} \widetilde{\mathbf{H}}(\mathbf{r}) \\ \nabla \times \widetilde{\mathbf{H}}(\mathbf{r})=-i \omega \varepsilon_{1} \widetilde{\mathbf{E}}(\mathbf{r})\end{array}\right\} \quad \mathbf{r} \in V_{\mathrm{EXT}}$, 


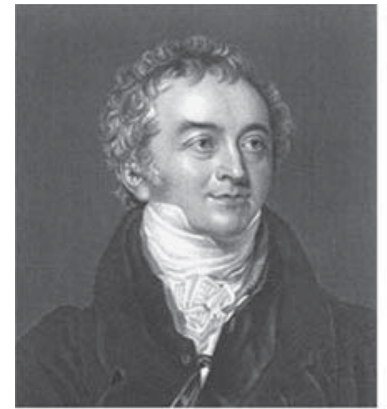

Thomas Young

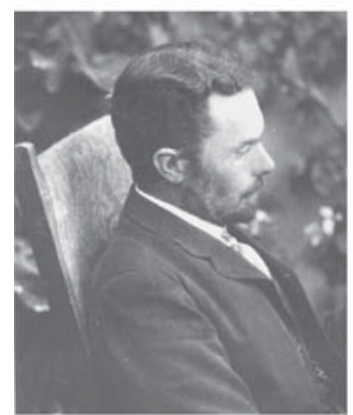

Oliver Heaviside

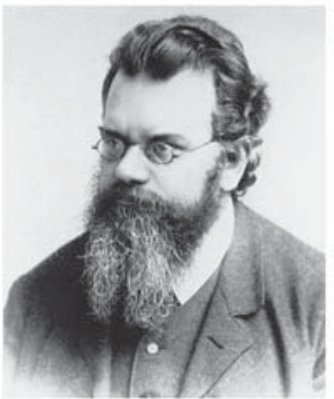

Ludwig Boltzmann

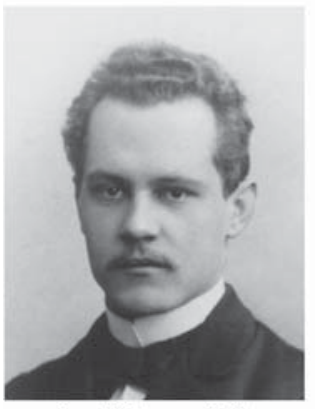

Arnold Sommerfeld

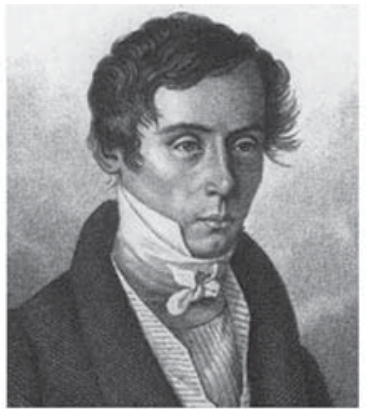

Augustin Jean Fresnel

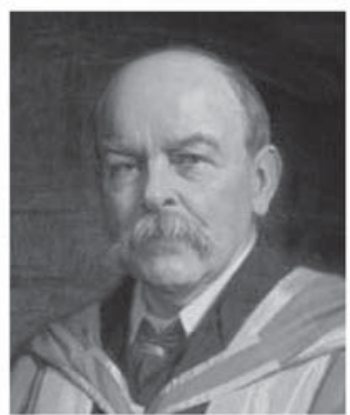

John Henry Poynting

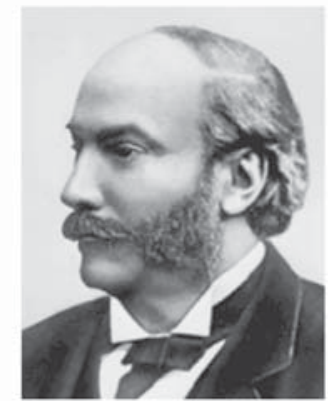

John William Strutt

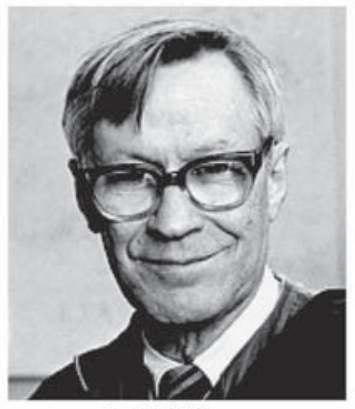

David S. Saxon

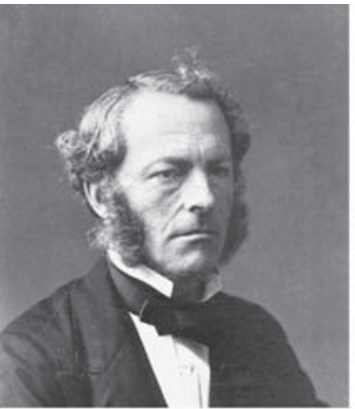

Sir George Gabriel Stokes

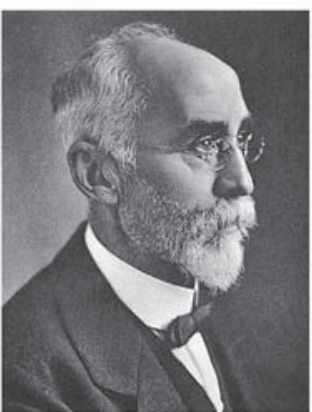

Hendrik Lorentz

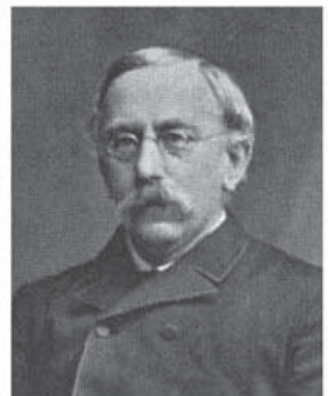

Ludvig Valentin Lorenz

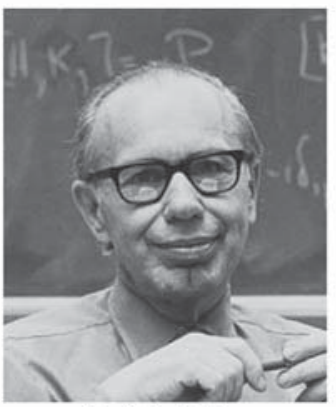

Leslie L. Foldy

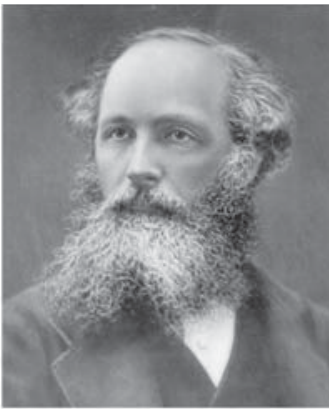

James Clerk Maxwell

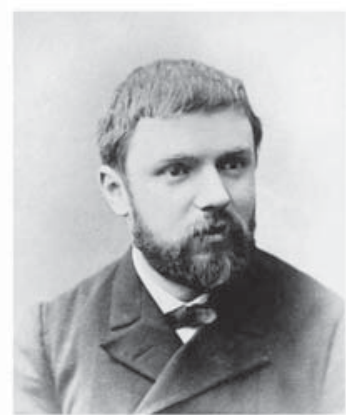

Jules Henri Poincaré

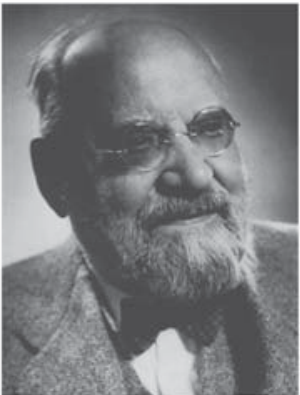

Gustav Mie

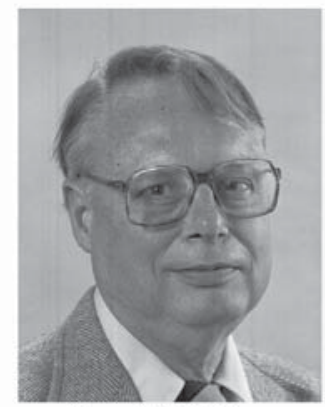

Kenneth M. Watson

Fig. 16. Scientists who had contributed (directly or indirectly) to the microphysical disciplines of directional radiometry and radiative transfer.

$\left.\begin{array}{l}\nabla \times \widetilde{\mathbf{E}}(\mathbf{r})=i \omega \mu_{0} \widetilde{\mathbf{H}}(\mathbf{r}) \\ \nabla \times \widetilde{\mathbf{H}}(\mathbf{r})=-i \omega \varepsilon_{2}(\mathbf{r}, \omega) \widetilde{\mathbf{E}}(\mathbf{r})\end{array}\right\} \quad \mathbf{r} \in V_{\mathrm{INT}}$.

In these equations, $V_{\text {INT }}$ is the cumulative "interior" volume occupied by the $N$ particles (Fig. 18):

$V_{\mathrm{INT}}=\bigcup_{i=1}^{N} V_{i}$ where $V_{i}$ is the volume occupied by the $i$ th particle; $V_{\mathrm{EXT}}$ is the infinite exterior region such that $V_{\text {INT }} \cup V_{\text {EXT }}=\Re^{3}$, where $\mathfrak{R}^{3}$ denotes the entire three-dimensional space; the host medium and the scattering object are assumed to be nonmagnetic; $\mu_{0}$ is the permeability of a vacuum; $\varepsilon_{1}$ is the real-valued electric permittivity of the host medium; and $\varepsilon_{2}(\mathbf{r}, \omega)$ is the (potentially coordinate-dependent) complex permittivity of the $N$-particle object. 


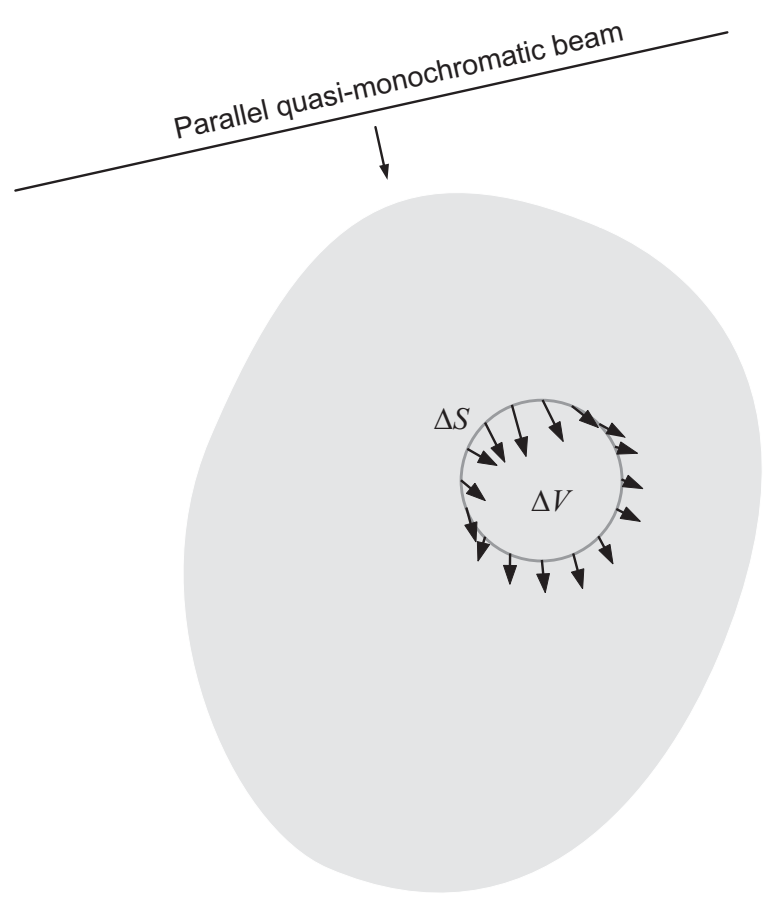

Fig. 17. Time-averaged energy budget of a volume element $\Delta V$ of a random particulate cloud bounded by the closed surface $\Delta S$. The arrows represent the distribution of $\langle\langle\mathbf{S}(\mathbf{r}, t)\rangle\rangle$ over the boundary $\Delta S$.

The corresponding boundary conditions read

$\left.\hat{\mathbf{n}} \times\left[\widetilde{\mathbf{E}}_{1}(\mathbf{r})-\widetilde{\mathbf{E}}_{2}(\mathbf{r})\right]=\mathbf{0}\right\}$

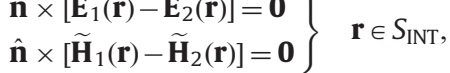

where $\mathbf{0}$ is a zero vector, the subscripts 1 and 2 correspond to the exterior and interior sides of the composite boundary $S_{\text {INT }}$ of the $N$-particle object, respectively, and $\hat{\mathbf{n}}$ is the local outward normal to $S_{\text {INT. }}$ In agreement with Eq. (15), $S_{\text {INT }}$ is the union of the closed surfaces of the $N$ particles

$S_{\mathrm{INT}}=\cup_{i=1}^{N} S_{i}$

Let us assume that the total field $\{\widetilde{\mathbf{E}}(\mathbf{r}), \widetilde{\mathbf{H}}(\mathbf{r})\}$ everywhere in space can be represented by a vector superposition of a plane-wave component (traditionally called the "incident field"; superscript "inc") propagating in the direction of the unit vector $\hat{\mathbf{n}}^{\text {inc }}$ and a "scattered field" (superscript "sca"):

$\widetilde{\mathbf{E}}(\mathbf{r})=\widetilde{\mathbf{E}}_{0}^{\text {inc }} \exp \left(i k_{1} \hat{\mathbf{n}}^{\text {inc }} \cdot \mathbf{r}\right)+\widetilde{\mathbf{E}}^{\text {sca }}(\mathbf{r})$,

$\widetilde{\mathbf{H}}(\mathbf{r})=\sqrt{\frac{\varepsilon_{1}}{\mu_{0}}} \hat{\mathbf{n}}^{\text {inc }} \times \widetilde{\mathbf{E}}_{0}^{\text {inc }} \exp \left(i k_{1} \hat{\mathbf{n}}^{\text {inc }} \cdot \mathbf{r}\right)+\widetilde{\mathbf{H}}^{\text {sca }}(\mathbf{r})$,

where $k_{1}=\omega\left(\varepsilon_{1} \mu_{0}\right)^{1 / 2}$ is the wave number of the exterior region. In addition, we postulate that the scattered field satisfies the following condition at infinity:

$\lim _{r \rightarrow \infty}\left\{\sqrt{\mu_{0}} \mathbf{r} \times \widetilde{\mathbf{H}}^{\text {sca }}(\mathbf{r})+r \sqrt{\varepsilon_{1}} \widetilde{\mathbf{E}}^{\text {sca }}(\mathbf{r})\right\}=\mathbf{0}$,

where $r=|\mathbf{r}|$ is the distance from the observation point to the fixed origin $O$ located near the "geometrical center" of the $N$-particle group (Fig. 18).
The curl Eqs. (13) and (14) supplemented by the boundary conditions (16), the decomposition (18), and the asymptotic condition (19) constitute the standard electromagnetic scattering problem for plane-wave illumination.

The above formulation of the standard electromagnetic scattering problem is hardly useful unless this problem has a solution and unless this solution is unique. To the best of my knowledge, the existence and the uniqueness of the solution of the standard scattering problem have been proven only in some particular cases. However, the thorough analysis by Claus Müller [160] demonstrates the fundamental importance of imposing both the boundary conditions and the asymptotic condition at infinity. It is, therefore, reasonable to assume that the standard scattering problem does have a solution, this solution being unique.

The pioneers of the theory of electromagnetic scattering by particles John William Strutt, Lord Rayleigh (1842-1919), Ludvig Valentin Lorenz (1829-1891), and Gustav Mie (1868-1957) considered it to be intuitively obvious that the "physically relevant" solution of the MMEs must involve the scattered field in the form of an outgoing spherical wave. Arnold Sommerfeld (1868-1951) was the first to realize in 1912 that the very uniqueness of the solution of an open-space scattering problem depends on the explicit postulation of an asymptotic condition at infinity [161]. The history of this so-called Sommerfeld radiation condition as well as its extensions and modifications are described in Ref. [162]. In the specific case of three-dimensional electromagnetic scattering by finite objects, the Sommerfeld radiation condition takes the form of Eq. (19) and is traditionally called the Silver-Müller radiation condition [160,163].

While the standard scattering problem is intentionally formulated for the incident field in the form of a plane

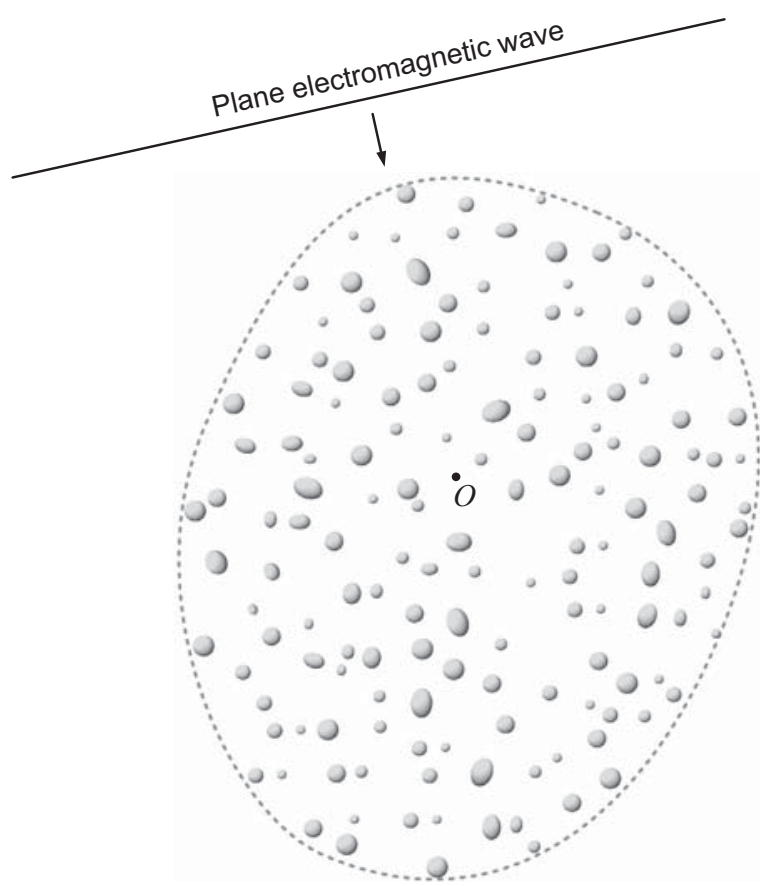

Fig. 18. A fixed $N$-particle group illuminated by a plane electromagnetic wave. 
electromagnetic wave, its actual range of relevance is much wider since the MMEs, the boundary conditions, and the radiation condition are all linear in the electric and magnetic fields. This implies that if $\left\{\widetilde{\mathbf{E}}^{\prime}, \widetilde{\mathbf{H}}^{\prime}\right\}$ and $\left\{\widetilde{\mathbf{E}}^{\prime \prime}, \widetilde{\mathbf{H}}^{\prime \prime}\right\}$ are the solutions of the standard problem corresponding to the incident plane waves $\left\{\left(\widetilde{\mathbf{E}}^{\text {inc }}\right)^{\prime},\left(\widetilde{\mathbf{H}}^{\text {inc }}\right)^{\prime}\right\}$ and $\left\{\left(\widetilde{\mathbf{E}}^{\text {inc }}\right)^{\prime \prime},\left(\widetilde{\mathbf{H}}^{\text {inc }}\right)^{\prime \prime}\right\}$, respectively, then $\left\{\widetilde{\mathbf{E}}^{\prime}+\widetilde{\mathbf{E}}^{\prime \prime}, \widetilde{\mathbf{H}}^{\prime}+\widetilde{\mathbf{H}}^{\prime \prime}\right\}$ is the unique solution of the boundary-value scattering problem for the incident field given by $\left\{\left(\widetilde{\mathbf{E}}^{\text {inc }}\right)^{\prime}+\left(\widetilde{\mathbf{E}}^{\text {inc }}\right)^{\prime \prime},\left(\widetilde{\mathbf{H}}^{\text {inc }}\right)^{\prime}+\left(\widetilde{\mathbf{H}}^{\text {inc }}\right)^{\prime \prime}\right\}$. Hence solutions of the standard scattering problem can be used to obtain the solution of a more general scattering problem provided that the corresponding incident field can be expanded in plane electromagnetic waves.

\subsection{Volume integral equation and Lippmann-Schwinger equation}

Although the standard scattering problem is stated as a boundary-value problem for the differential frequencydomain MMEs, it is often convenient to invoke an equivalent integral-equation formulation. Specifically, the socalled volume integral equation (VIE) expresses the total electric field everywhere in space in terms of the incident electric field and the total electric field in the interior volume of the $N$-particle group. The VIE is a straightforward consequence of Eqs. (13)-(19) and reads

$\widetilde{\mathbf{E}}(\mathbf{r})=\widetilde{\mathbf{E}}^{\text {inc }}(\mathbf{r})+k_{1}^{2} \int_{V_{\mathrm{INT}}} \mathrm{d}^{3} \mathbf{r}^{\prime}\left[m^{2}\left(\mathbf{r}^{\prime}\right)-1\right] \stackrel{\leftrightarrow}{G}\left(\mathbf{r}, \mathbf{r}^{\prime}\right) \cdot \widetilde{\mathbf{E}}\left(\mathbf{r}^{\prime}\right), \quad \mathbf{r} \in \Re^{3}$,

where $m(\mathbf{r})=k_{2}(\mathbf{r}) / k_{1}$ is the refractive index of the interior relative to that of the exterior, $k_{2}(\mathbf{r})=\omega\left[\varepsilon_{2}(\mathbf{r}, \omega) \mu_{0}\right]^{1 / 2}$ is the wave number of the interior region,

$\overleftrightarrow{G}\left(\mathbf{r}, \mathbf{r}^{\prime}\right)=\left(\overleftrightarrow{I}+\frac{1}{k_{1}^{2}} \nabla \otimes \nabla\right) \frac{\exp \left(i k_{1}\left|\mathbf{r}-\mathbf{r}^{\prime}\right|\right)}{4 \pi\left|\mathbf{r}-\mathbf{r}^{\prime}\right|}, \quad \mathbf{r}, \mathbf{r}^{\prime} \in \Re^{3}$

is the free-space dyadic Green's function, $\stackrel{\leftrightarrow}{I}$ is the identity dyadic, and $\otimes$ is the dyadic product sign. A major advantage of the VIE is that the boundary conditions (16) and the radiation condition (19) are built into it rather than serve as additional constraints imposed on the physically relevant solution of the differential frequency-domain MMEs. The electric field found by solving the VIE can be substituted in the second formulas of Eqs. (13) and (14) in order to find the magnetic field everywhere in space.

The dyadic Green's function was introduced in 1950 by Harold Levine and Julian Schwinger [164]. It belongs to the general class of functions first studied by the self-taught English mathematician (and professional miller) George Green (1793-1841) in his initially obscure, but now famous Essay of 1828 privately printed at Green's own expense in the form of a booklet [165]. Typically, a Green's function is an integral kernel that can be used to solve an inhomogeneous differential equation defined on a domain, with specified boundary conditions. The Essay was enthusiastically popularized by William Thomson, Lord Kelvin who arranged its re-publication in Journal für die reine und angewandte Mathematik (also known as Crelle's Journal) in 1850-1854. Green's approach was further developed into an efficient mathematical tool by Oliver Heaviside.

Note that the majority of derivations of the VIE published in monographs and journal papers, including the original derivation by David Saxon (1920-2005) in 1955 [166], do not invoke explicitly the boundary conditions (16) as the prerequisite of the uniqueness of solution of the standard scattering problem. As such, they are applicable only to "fuzzy" scattering objects without distinct boundaries. This means that the refractive index is implicitly assumed to depend on spatial coordinates continuously throughout the entire space rather than being discontinuous across particle surfaces. A complete derivation explicitly relying on Eq. (16) and thereby applicable to scattering objects with distinct boundaries is given in Refs. $[140,167]$.

The VIE can be perceived as the very embodiment of the concept of electromagnetic scattering. Indeed, it shows that in the absence of the scattering object, i.e., when $m\left(\mathbf{r}^{\prime}\right) \equiv 1$, the total field is identically equal to the incident field. The presence of the object changes the total field, which means that the scattered field can be defined as the difference between the total fields in the presence and in the absence of the object. Furthermore, the VIE makes it obvious that the incident field is not modified by the presence of the object and, contrary to the widespread misconception, is not "transformed into the scattered field" [168].

It is convenient to express the scattered field mathematically in terms of the incident field as follows:

$\widetilde{\mathbf{E}}^{\mathrm{sca}}(\mathbf{r})=\int_{V_{\mathrm{INT}}} \mathrm{d}^{3} \mathbf{r}^{\prime} \stackrel{\leftrightarrow}{G}\left(\mathbf{r}, \mathbf{r}^{\prime}\right) \cdot \int_{V_{\mathrm{INT}}} \mathrm{d}^{3} \mathbf{r}^{\prime \prime} \stackrel{\leftrightarrow}{T}\left(\mathbf{r}^{\prime}, \mathbf{r}^{\prime \prime}\right) \cdot \widetilde{\mathbf{E}}^{\text {inc }}\left(\mathbf{r}^{\prime \prime}\right), \quad \mathbf{r} \in \Re^{3}$,

where $\stackrel{\leftrightarrow}{T}$ is the so-called dyadic transition operator (DTO). Substituting Eq. (22) in Eq. (20) yields the following integral equation for $\stackrel{\leftrightarrow}{T}$ :

$$
\begin{aligned}
\stackrel{\leftrightarrow}{T}\left(\mathbf{r}, \mathbf{r}^{\prime}\right)= & k_{1}^{2}\left[m^{2}(\mathbf{r})-1\right] \delta\left(\mathbf{r}-\mathbf{r}^{\prime}\right) \stackrel{\leftrightarrow}{I}+k_{1}^{2}\left[m^{2}(\mathbf{r})-1\right] \\
& \times \int_{V_{\mathrm{INT}}} \mathrm{d}^{3} \mathbf{r}^{\prime \prime} \stackrel{\leftrightarrow}{G}\left(\mathbf{r}, \mathbf{r}^{\prime \prime}\right) \cdot \stackrel{\leftrightarrow}{T}\left(\mathbf{r}^{\prime \prime}, \mathbf{r}^{\prime}\right), \quad \mathbf{r}, \mathbf{r}^{\prime} \in V_{\text {INT }}
\end{aligned}
$$

Equations of this type first were introduced in 1950 by Bernard A. Lippmann and Julian Schwinger in the quantum theory of scattering [169] and are traditionally called Lippmann-Schwinger equations [170,171]. The first derivation of Eq. (23) in the electromagnetic-scattering case was given by Leung Tsang and Jin Au Kong in 1980 [172] (see also Refs. [173,174]).

A fundamental property of the DTO is that it is independent of the incident field and is a function of the scattering object only: it is fully defined by the distribution of the relative refractive index throughout $V_{\text {INT. }}$. It is this property that has made the concept of the DTO central to the theory of electromagnetic scattering by multi-particle groups. In particular, as discussed in the following subsection, it was used to derive the so-called Foldy equations (FEs) that eventually served as a natural precursor to the microphysical theory of radiative transfer in sparse particulate media. 


\subsection{Foldy equations}

If the scattering object is a multi-particle group, then it is very convenient to formally represent the total scattered field as a vector superposition of the "partial" fields contributed by the individual particles. In the case of an arbitrary $\mathrm{N}$-particle group, this cannot be done easily using the differential frequency-domain Maxwell equations, but is quite straightforward in the framework of the VIE formalism. Indeed, let us first re-write the VIE as follows:

$\widetilde{\mathbf{E}}(\mathbf{r})=\widetilde{\mathbf{E}}^{\text {inc }}(\mathbf{r})+\int_{\mathfrak{R}^{3}} \mathrm{~d}^{3} \mathbf{r}^{\prime} U\left(\mathbf{r}^{\prime}\right) \overleftrightarrow{G}\left(\mathbf{r}, \mathbf{r}^{\prime}\right) \cdot \widetilde{\mathbf{E}}\left(\mathbf{r}^{\prime}\right), \quad \mathbf{r} \in \Re^{3}$,

where the integration is performed over the entire space, the potential function $U(\mathbf{r})$ is given by

$U(\mathbf{r})=\sum_{i=1}^{N} U_{i}(\mathbf{r}), \quad \mathbf{r} \in \Re^{3}$,

and $U_{i}(\mathbf{r})$ is the potential function of the $i$ th-particle. The latter is given by

$U_{i}(\mathbf{r})=\left\{\begin{array}{cc}0, & \mathbf{r} \notin V_{i}, \\ k_{1}^{2}\left[m_{i}^{2}(\mathbf{r})-1\right], & \mathbf{r} \in V_{i},\end{array}\right.$

where

$m_{i}(\mathbf{r})=k_{2 i}(\mathbf{r}) / k_{1}$

is the refractive index of particle $i$ relative to that of the host medium. All position vectors originate at the common origin $O$ of the laboratory coordinate system (Fig. 18). It can then be shown that the total electric field everywhere in space is given by the following expression:

$\widetilde{\mathbf{E}}(\mathbf{r})=\widetilde{\mathbf{E}}^{\text {inc }}(\mathbf{r})+\sum_{i=1}^{N} \int_{V_{i}} \mathrm{~d}^{3} \mathbf{r}^{\prime} \stackrel{\leftrightarrow}{G}\left(\mathbf{r}, \mathbf{r}^{\prime}\right) \int_{V_{i}} \mathrm{~d}^{3} \mathbf{r}^{\prime \prime} \stackrel{\leftrightarrow}{T}_{i}\left(\mathbf{r}^{\prime}, \mathbf{r}^{\prime \prime}\right) \cdot \widetilde{\mathbf{E}}_{i}\left(\mathbf{r}^{\prime \prime}\right)$,

$\mathbf{r} \in \mathfrak{R}^{3}$,

where the total electric field $\widetilde{\mathbf{E}}_{i}(\mathbf{r})$ "exciting" particle $i$ is given by

$\widetilde{\mathbf{E}}_{i}(\mathbf{r})=\widetilde{\mathbf{E}}^{\mathrm{inc}}(\mathbf{r})+\sum_{j(\neq i)=1}^{N} \widetilde{\mathbf{E}}_{i j}^{\mathrm{exc}}(\mathbf{r})$

the $\widetilde{\mathbf{E}}_{i j}^{\mathrm{exc}}(\mathbf{r})$ are partial exciting fields given by

$\widetilde{\mathbf{E}}_{i j}^{\text {exc }}(\mathbf{r})=\int_{V_{i}} \mathrm{~d}^{3} \mathbf{r}^{\stackrel{\leftrightarrow}{G}}\left(\mathbf{r}, \mathbf{r}^{\prime}\right) \cdot \int_{V_{j}} \mathrm{~d}^{3} \mathbf{r}^{\prime \prime} \stackrel{\leftrightarrow}{T} j\left(\mathbf{r}^{\prime}, \mathbf{r}^{\prime \prime}\right) \cdot \widetilde{\mathbf{E}}_{j}\left(\mathbf{r}^{\prime \prime}\right), \quad \mathbf{r} \in V_{i}$,

and $\stackrel{\leftrightarrow}{T}_{i}$ is the $i$ th-particle DTO defined with respect to the laboratory coordinate system; it satisfies the following Lippmann-Schwinger equation:

$\overleftrightarrow{T}_{i}\left(\mathbf{r}, \mathbf{r}^{\prime}\right)=U_{i}(\mathbf{r}) \delta\left(\mathbf{r}-\mathbf{r}^{\prime}\right) \overleftrightarrow{I}+U_{i}(\mathbf{r}) \int_{V_{i}} \mathrm{~d}^{3} \mathbf{r}^{\prime \prime} \overleftrightarrow{G}\left(\mathbf{r}, \mathbf{r}^{\prime \prime}\right)$

$\stackrel{\leftrightarrow}{T}_{i}\left(\mathbf{r}^{\prime \prime}, \mathbf{r}^{\prime}\right), \quad \mathbf{r}, \mathbf{r}^{\prime} \in V_{i}$

Importantly, each $\stackrel{\leftrightarrow}{T}_{i}$ is computed individually, as if all the other particles did not exist.

A simplified version of Eqs. (28)-(31) was proposed heuristically in 1945 by Leslie L. Foldy (1919-2001) [175] to describe scattering of scalar waves by a system of widely separated isotropic point centers. Various applications of Foldy's approach were discussed in 1951 by Melvin Lax [176]. A rigorous derivation of the quantum-mechanical version of the FEs from the Schrödinger equation was given in 1953 by Kenneth M. Watson [177] (see also Section 11.3 of Ref. [170]). The case of electromagnetic scattering by a group of widely separated electrons was considered by Watson in 1969 [178]. The general electromagnetic FEs in the form of Eqs. (28)-(31) were derived by Tsang and Kong in 1980 [172] (see also Ref. [173] in which the electromagnetic FEs were derived using Watson's approach).

The FEs (28)-(31) (also known as Foldy-Lax equations $[136,179])$ are mathematically equivalent to the VIE (20) and hardly offer computational advantages. However, there are two factors which make them important. First, they allow one to introduce the Neumann expansion of the total field playing a key role in the microphysical theory of radiative transfer. Second, as discussed in the following subsection, they can be used to incorporate the notion of the single-particle far field in the computation of the near field of a multi-particle group.

Let us first define the $i$ th potential dyadic centered at the origin of the laboratory reference frame according to

$\stackrel{\leftrightarrow}{U}_{i}\left(\mathbf{r}, \mathbf{r}^{\prime}\right)=U_{i}(\mathbf{r}) \delta\left(\mathbf{r}-\mathbf{r}^{\prime}\right) \stackrel{\leftrightarrow}{I}$

and introduce the following operator notation:

$\hat{U}=\sum_{i=1}^{N} \hat{U}_{i}$

$\hat{B} E=\int \mathrm{d}^{3} \mathbf{r}^{\prime} \stackrel{\leftrightarrow}{B}\left(\mathbf{r}, \mathbf{r}^{\prime}\right) \cdot \widetilde{\mathbf{E}}\left(\mathbf{r}^{\prime}\right)$

Iterating Eqs. (29) and (30) yields

$$
\begin{aligned}
E_{i}= & E^{\mathrm{inc}}+\sum_{j(\neq i)=1}^{N} \hat{G} \hat{T}_{j} E^{\mathrm{inc}}+\sum_{j(\neq i)=1}^{N} \sum_{l(\neq j)=1}^{N} \hat{G} \hat{T}_{j} \hat{G} \hat{T}_{l} E^{\mathrm{inc}} \\
& +\sum_{j(\neq i)=1}^{N} \sum_{l \neq j)=1}^{N} \sum_{m(\neq l)=1}^{N} \hat{G} \hat{T}_{j} \hat{G} \hat{T}_{l} \hat{G} \hat{T}_{m} E^{\mathrm{inc}}+\cdots,
\end{aligned}
$$

whereas the substitution of Eq. (35) in Eq. (28) results in the following expansion, traditionally called in mathematics the Neumann series:

$$
\begin{aligned}
E= & E^{\mathrm{inc}}+\sum_{i=1}^{N} \hat{G} \hat{T}_{i} E^{\mathrm{inc}}+\sum_{i=1}^{N} \sum_{j(\neq i)=1}^{N} \hat{G} \hat{T}_{i} \hat{G} \hat{T}_{j} E^{\mathrm{inc}} \\
& +\sum_{i=1}^{N} \sum_{j(\neq i)=1}^{N} \sum_{l(\neq j)=1}^{N} \hat{G} \hat{T}_{i} \hat{G} \hat{T}_{j} \hat{G} \hat{T}_{l} E^{\mathrm{inc}}+\cdots
\end{aligned}
$$

It is easily seen that the Neumann series is fundamentally based on the fact that $\stackrel{\leftrightarrow}{T}_{i}$ for each $i$ is an individual property of the $i$ th particle computed as if this particle were alone rather than a member of the group. As a consequence, there has been a tendency to characterize the Neumann expansion (36) as describing "multiple scattering" by the $\mathrm{N}$-particle group. It is important to recognize, however, that although the notion of multiple scattering, as embodied by Eq. (36), can be a useful mathematical abstraction, it is not a real physical phenomenon wherein the incident light is scattered sequentially by one, two, three or more particles before reaching the observation point $[168,180]$. In other words, one can speak of multi-particle sequences contributing the various terms on the right-hand side of Eq. (36), but not of actual "multiple-scattering paths" or "multiple-scattering trajectories". 
The concept of the Neumann expansion (also known as the Liouville-Neumann or Liouville-Neumann-Volterra series) was introduced by the French mathematician Joseph Liouville (1809-1882) in 1837 and by the German mathematician Carl Gottfried Neumann (1832-1925) thirty years later. The Italian mathematician Vito Volterra (1860-1940) used this expansion in his general theory of integral equations as a technique for solving Fredholm and Volterra equations of the second kind, wherein the unknown function is expanded in a power series in terms of so-called iterated kernels. The method is applicable whenever the series converges [181,182]. In quantum theory of particle scattering, an expansion of the type (36) is often called the Born series [170,171].

\subsection{Far-field Foldy equations}

The reader should recognize that the formulation of electromagnetic scattering in Sections 4.1-4.4 has been quite general. Although we chose to speak of the scattering object in the form of a fixed group of $N$ "distinct" nonoverlapping particles, Eqs. (13)-(36) apply to any morphology of the scattering object as long as the entire object is finite. For example, we could consider a single ellipsoid and subdivide it into an arbitrary set of $N$ non-overlapping volume elements, in which case the FEs would still hold. This demonstrates again that the concept of multiple frequency-domain scattering, as represented by the Neumann expansion (36), is nothing more than a mathematical abstraction [180].

As already mentioned, the FEs offer no computational advantages over the VIE in the case of an arbitrary fixed scattering object. However, they become indispensable if the scattering object is a large, sparse, random cloud of particles, in which case the Neumann expansion (36) helps accommodate in a very straightforward analytical fashion two main morphological traits of the object: large particleto-particle distances and statistically uncorrelated particle positions.

Specifically, let us first assume that:

- the particles forming the group are separated widely enough that each of them is located in the far zones of all the other particles, and

- the observation point is located in the far zone of any particle in the group (but not necessarily in the far zone of the entire group).

As a consequence, each partial exciting field $\widetilde{\mathbf{E}}_{i j}^{\text {exc }}(\mathbf{r})$ in Eq. (29) becomes an outgoing spherical wavelet centered at particle $j$, while the integral FEs are converted into algebraic far-field FEs. The latter imply that the total field at any observation point located sufficiently far from any particle in the sparse multi-particle group is the superposition of the incident plane wave and $N$ partial spherical wavelets contributed by the $N$ particles. The observation point is not required to be in the far zone of the entire group. It can be anywhere in space as long as it resides in the far zones of all the particles forming the group.
It is important to recognize that even though each component of the cumulative scattered field given by the far-field FEs is a transverse electromagnetic wave, the scattered field itself is not, in general, a transverse electromagnetic wave. In other words, the far-field FEs describe the near field of the multi-particle group despite the underlying assumption that the observation point is located in the far zone of any constituent particle. The cumulative scattered field becomes a transverse electromagnetic wave only if the observation point is located in the far zone of the entire group.

The corresponding far-field Neumann expansion of the total electric field also becomes purely algebraic and takes the form

$$
\begin{aligned}
\widetilde{\mathbf{E}}= & \widetilde{\mathbf{E}}^{\text {inc }}+\sum_{i=1}^{N} \stackrel{\leftrightarrow}{B}_{r i 0} \cdot \widetilde{\mathbf{E}}_{i}^{\text {inc }}+\sum_{i=1}^{N} \sum_{j(\neq i)=1}^{N} \stackrel{\leftrightarrow}{B}_{r i j} \cdot \stackrel{\leftrightarrow}{B}_{i j 0} \cdot \widetilde{\mathbf{E}}_{j}^{\text {inc }} \\
& +\sum_{i=1}^{N} \sum_{j(\neq i)=1}^{N} \sum_{l(\neq j)=1}^{N} \stackrel{\leftrightarrow}{B}_{r i j} \cdot \stackrel{\leftrightarrow}{B}_{i j l} \cdot \overleftrightarrow{B}_{j l 0} \cdot \widetilde{\mathbf{E}}_{l}^{\text {inc }} \\
& +\sum_{i=1}^{N} \sum_{j(\neq i)=1}^{N} \sum_{l(\neq j)=1}^{N} \sum_{m(\neq l)=1}^{N} \stackrel{\leftrightarrow}{B}_{r i j} \cdot \stackrel{\leftrightarrow}{B}_{i j l} \cdot \stackrel{\leftrightarrow}{B}_{j l m} \cdot \stackrel{\leftrightarrow}{B}_{l m 0} \cdot \widetilde{\mathbf{E}}_{m}^{\text {inc }}+\cdots,
\end{aligned}
$$

where each dyadic $\stackrel{\leftrightarrow}{B}_{i j l}$ describes far-field scattering by the $j$ th particle. It is Eq. (37) that represents the starting point in the development of the microphysical theory of radiative transfer.

The far-field electromagnetic FEs were introduced heuristically by Anatoly Borovoi for a group of identical spherical particles in 1966 [122]. The first rigorous derivation from the general electromagnetic FEs (28)-(31) appeared in 1984 [173] (see also Refs. [137,183]).

\subsection{The Twersky approximation}

The terms with $j=i$ and $l=j$ in the triple summation on the right-hand side of the far-field Neumann expansion (37) are excluded, but the terms with $l=i$ are not. Therefore, we can decompose this summation as follows:

$$
\begin{aligned}
& \sum_{i=1}^{N} \sum_{j=1}^{N} \sum_{l=1}^{N} \stackrel{\leftrightarrow}{B}_{r i j} \cdot \stackrel{\leftrightarrow}{B}_{i j l} \cdot \stackrel{\leftrightarrow}{B}_{j l 0} \cdot \widetilde{\mathbf{E}}_{l}^{\text {inc }} \\
& j \neq i \quad l \neq j \\
& =\sum_{i=1}^{N} \sum_{\substack{j=1 \\
j \neq i}}^{N} \sum_{\substack{l=1 \\
l \neq i}}^{N} \stackrel{\leftrightarrow}{B}_{r i j} \cdot \stackrel{\leftrightarrow}{B}_{i j l} \cdot \stackrel{\leftrightarrow}{B}_{j l 0} \cdot \widetilde{\mathbf{E}}_{l}^{\text {inc }} \\
& l \neq j \\
& +\sum_{i=1}^{N} \sum_{j=1}^{N} \stackrel{\leftrightarrow}{B}_{r i j} \cdot \stackrel{\leftrightarrow}{B}_{i j i} \cdot \stackrel{\leftrightarrow}{B}_{j i 0} \cdot \widetilde{\mathbf{E}}_{i}^{\text {inc }}
\end{aligned}
$$

Higher-order summations in Eq. (37) can be decomposed similarly. Thus, the total field at an observation point $\mathbf{r}$ is composed of the incident field, one-particle contributions, and contributions from multi-particle sequences that can be divided into two groups. The first one includes all the terms contributed by self-avoiding multi-particle sequences, whereas the second group includes all the terms corresponding to multi-particle sequences that involve a particle more than once. The approximation introduced in 1964 by Victor Twersky [184] (for the case 
of scattering of scalar waves) results in a drastic simplification by neglecting the terms belonging to the second group and retaining only the terms from the first group:

$$
\begin{aligned}
& \widetilde{\mathbf{E}} \approx \widetilde{\mathbf{E}}^{\mathrm{inc}}+\sum_{i=1}^{N} \stackrel{\leftrightarrow}{B}_{r i 0} \cdot \widetilde{\mathbf{E}}_{i}^{\mathrm{inc}}+\sum_{i=1}^{N} \sum_{\substack{j=1 \\
j \neq i}}^{N} \overleftrightarrow{B}_{r i j} \cdot \overleftrightarrow{B}_{i j 0} \cdot \widetilde{\mathbf{E}}_{j}^{\text {inc }} \\
& +\sum_{i=1}^{N} \sum_{\substack{j=1 \\
j \neq i}}^{N} \sum_{\substack{l=1 \\
l \neq i}}^{N} \stackrel{\leftrightarrow}{B}_{r i j} \cdot \stackrel{\leftrightarrow}{B}_{i j l} \cdot \stackrel{\leftrightarrow}{B}_{j l 0} \cdot \widetilde{\mathbf{E}}_{l}^{\text {inc }} \\
& l \neq j \\
& +\sum_{i=1}^{N} \sum_{j=1}^{N} \sum_{l=1}^{N} \sum_{m=1}^{N} \stackrel{\leftrightarrow}{B}_{r i j} \cdot \stackrel{\leftrightarrow}{B}_{i j l} \cdot \overleftrightarrow{B}_{j l m} \cdot \stackrel{\leftrightarrow}{B}_{l m 0} \cdot \widetilde{\mathbf{E}}_{m}^{\text {inc }}+\cdots \\
& j \neq i \quad l \neq i \quad m \neq i \\
& l \neq j \quad m \neq j
\end{aligned}
$$

It can be shown indeed that in the limit $N \rightarrow \infty$ the Twersky approximation accounts for the overwhelming majority of multi-particle sequences, which implies that it can be expected to yield rather accurate results provided that the number of particles in the group is sufficiently large.

\subsection{The Poynting-Stokes tensor and dyadic correlation function}

The Twersky expansion (39) coupled with the first equation of Eq. (13) can be used to write an extended expression for the time average of the complex Poynting vector $\widetilde{\mathbf{S}}(\mathbf{r})=\frac{1}{2} \widetilde{\mathbf{E}}(\mathbf{r}) \times \widetilde{\mathbf{H}}^{*}(\mathbf{r})$, where the asterisk denotes a complex-conjugate value. However, doing that does not enable one to contract the resulting infinite set of nested summations and obtain a closed-form equation amenable to efficient analytical or numerical solution. The ultimate reason for that is the following inescapable fact: different combinations of electric and magnetic field vectors can yield the same Poynting vector. This means that forming the vector product of the electric and magnetic field vectors results in a quantity that does not carry unique information about the participating fields. As a consequence, the Poynting vector cannot be used to describe the phenomenon of electromagnetic scattering by, for example, expressing the Poynting vector of the total field in that of the incident field.

It is also customary to describe electromagnetic scattering in the far zone of a finite object in terms of the $4 \times 4$ Stokes phase matrix using the 4-element Stokes column vector as the primary descriptor of polarization. However, this formalism can be applied only to transverse (e.g., plane or spherical) electromagnetic waves, whereas the total electromagnetic field in the near zone of any object (e.g., at any observation point inside a multi-particle group) is never a transverse wave.

It was shown by the author in 2010 [116] (see also Ref. [140]) that instead of attempting to calculate directly the time-averaged Poynting vector or Stokes column vector, one should first calculate the time average of the Poytning-Stokes tensor (PST) defined as the dyadic product of the electric and magnetic field vectors:

$$
\stackrel{\leftrightarrow}{P}(\mathbf{r})=\frac{1}{2} \widetilde{\mathbf{H}}(\mathbf{r}) \otimes \widetilde{\mathbf{E}}^{*}(\mathbf{r})
$$

It is fundamentally important that by its very construct, the PST is applicable to an arbitrary electromagnetic field (including the near field) and thus can be used to find both the Poynting vector and, whenever applicable, relevant optical observables such as the Stokes parameters.

The PST involves both the electric and the magnetic field at the observation point $\mathbf{r}$. It is sometimes convenient to have an alternative representation involving only the electric field. It can be verified easily that in the case of nonmagnetic materials, Eq. (40) everywhere in space can be written in the form

$$
\stackrel{\leftrightarrow}{P}(\mathbf{r})=\left.\frac{1}{2 i \omega \mu_{0}}\left[\nabla_{\mathbf{r}^{\prime}} \times \overleftrightarrow{C}\left(\mathbf{r}^{\prime}, \mathbf{r}\right)\right]\right|_{\mathbf{r}^{\prime}=\mathbf{r}}
$$

where

$$
\overleftrightarrow{C}\left(\mathbf{r}^{\prime}, \mathbf{r}\right)=\widetilde{\mathbf{E}}\left(\mathbf{r}^{\prime}\right) \otimes \widetilde{\mathbf{E}}^{*}(\mathbf{r})
$$

is the so-called dyadic correlation function involving the electric field at two different points in space. The subscript $\mathbf{r}^{\prime}$ means that the $\nabla$ operator acts only on $\widetilde{\mathbf{E}}\left(\mathbf{r}^{\prime}\right)$.

Using the Twersky expansion (39), we can formulate the Twersky approximation for the dyadic correlation function diagrammatically according to Fig. 19. The different terms entering the expanded expression inside the angular brackets on the right-hand side of this equation can be classified using the notation introduced in Fig. 20a. In this particular case, the upper and lower multi-particle sequences involve different particles. However, the two multi-particle sequences can involve one or more common particles, as shown in Fig. 20c-f by using the dashed connectors. Moreover, if the number of common particles in a diagram is two or more, then they can enter the upper and lower sequences in the same order, as in Fig. 20d, or in the reverse order, as in Fig. 20e. Finally, Fig. 20f gives an example of a mixed diagram wherein two common particles appear in the same order while two other common particles appear in the reverse order.

\subsection{Ergodicity}

So far we have been considering electromagnetic scattering by a fixed configuration of particles. Let us now assume that the sparse $\mathrm{N}$-particle group varies in time, but does it slowly enough that any significant (i.e., modifying the solution of the MMEs) changes of the group occur over time intervals $T_{\mathrm{v}}$ much longer than the period of time-harmonic oscillations of the electromagnetic field. Then the time average of the dyadic correlation function is formally defined by

$\left\langle\left\langle\overleftrightarrow{C}\left(\mathbf{r}^{\prime}, \mathbf{r} ; t\right)\right\rangle\right\rangle=\frac{1}{T} \int_{t-(T / 2)}^{t+(T / 2)} \mathrm{d} t^{\prime} \stackrel{\leftrightarrow}{C}\left(\mathbf{r}^{\prime}, \mathbf{r} ; t^{\prime}\right)$

where the time interval $T$ is much longer than $T_{\mathrm{v}}$. During this time interval the variable $\mathrm{N}$-particle group goes through an infinite sequence of evolving discrete states governed by relevant physical and chemical processes. Therefore, the right-hand side of Eq. (43) must be evaluated by: (i) tracing the temporal evolution of the physical state of the entire 


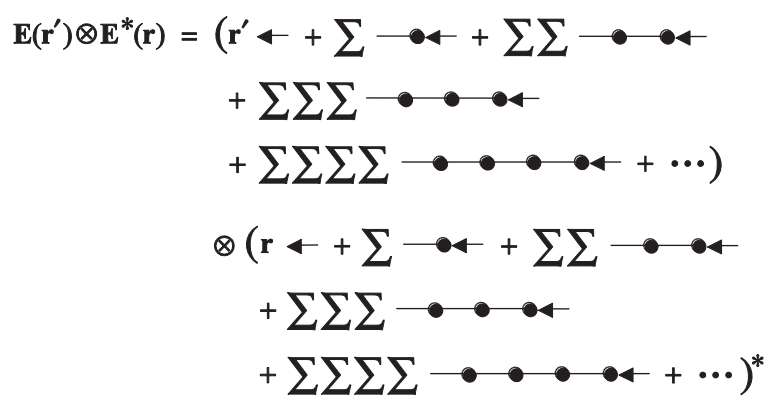

Fig. 19. The Twersky approximation for the dyadic correlation function. An arrow denotes the corresponding local incident field, while a dot denotes left multiplication by the corresponding $B$-dyadic.

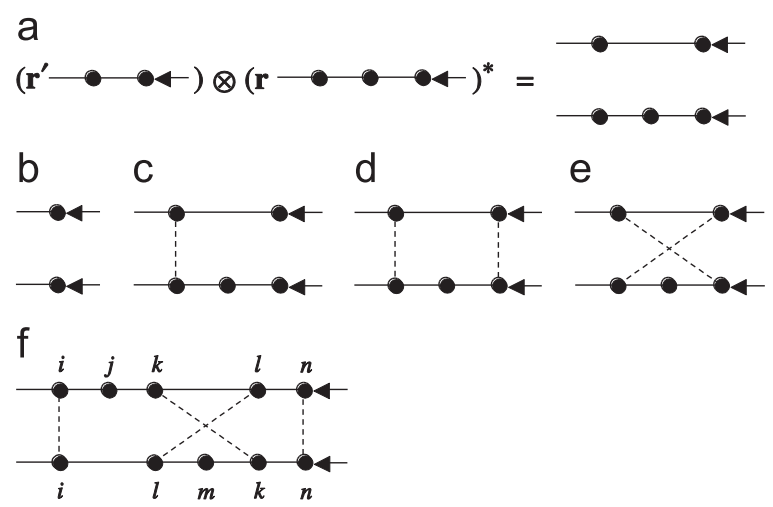

Fig. 20. Classification of different terms entering the Twersky approximation for the dyadic correlation function.

group (i.e., the complete set of particle coordinates and microphysical states), and (ii) computing $C\left(\mathbf{r}^{\prime}, \mathbf{r} ; t^{\prime}\right)$ for a sufficiently representative set of moments $t^{\prime} \in[t-T / 2, t+T / 2]$.

Although a computer implementation of this averaging procedure is not totally inconceivable, the direct use of the definition (43) in analytical derivations - such as the calculation of the time-averaged dyadic correlation function for a cloud of particles - typically leads to insurmountable technical difficulties. A much more practicable approach is based on the assumption that the $\mathrm{N}$ particle group is statistically random and sufficiently variable in time while the time interval $T$ is sufficiently long that averaging $\overleftrightarrow{C}\left(\mathbf{r}^{\prime}, \mathbf{r} ; t^{\prime}\right)$ over this interval is essentially equivalent to averaging $\overleftrightarrow{C}\left(\mathbf{r}^{\prime}, \mathbf{r} ; \psi\right)$ over an appropriate analytical probability distribution of the physical state $\psi$ of the group. In other words, it is assumed that averaging over time for one specific realization of the random scattering process is equivalent to ensemble averaging. The equivalence of the time and ensemble averages is called ergodicity.

The ergodic hypothesis was introduced by James Clerk Maxwell and Ludwig Boltzmann (1844-1906) as a basic underlying principle of statistical mechanics and kinetic theory. The mathematical foundation of the ergodic theory, its relation to the famous Poincare recurrence theorem [185], and applications to statistical physics are discussed in Refs. [186-188].

\subsection{Time-averaged Poynting vector}

According to the preceding subsection, instead of calculating the average $\left\langle\left\langle\overleftrightarrow{C}\left(\mathbf{r}^{\prime}, \mathbf{r} ; t\right)\right\rangle\right\rangle$, one can calculate the average $\left\langle\overleftrightarrow{C}\left(\mathbf{r}^{\prime}, \mathbf{r} ; \mathbf{R}, \xi\right)\right\rangle_{\mathbf{R}, \xi}$, where $\mathbf{R}$ denotes the complete set of particle coordinates and $\xi$ denotes the complete set of particle microphysical states. Although this problem remains very complex in general, it becomes manageable upon further assuming that:

1. The position and microphysical state of each particle are statistically independent of each other and of those of all the other particles.

2. The microphysical states of all the particles have the same statistical characteristics.

3. The spatial distribution of the particles throughout the medium is completely random and statistically uniform.

4. All diagrams with crossing connectors in the diagrammatic expansion of the dyadic correlation function (cf. Figs. 19 and 20) can be ignored. This is the essence of the so-called ladder approximation [125].

All intermediate steps of the subsequent analytical derivation are detailed in Ref. [140]. Each $\stackrel{\leftrightarrow}{B}$-dyadic in the Twersky expansion (39) includes a complex exponential factor of the type $\exp \left(i k_{1} R\right)$, where $R$ can be the distance between two particles or the distance from a particle to the observation point. Analytical averaging of these rapidly oscillating exponentials over uncorrelated and random particle positions leads to dramatic simplifications and as such is at the very heart of the microphysical RTT. The final expression for the time-averaged Poynting vector at an internal observation point $\mathbf{r}$ is as follows:

$\langle\langle\mathbf{S}(\mathbf{r}, t)\rangle\rangle=\operatorname{Re}\langle\widetilde{\mathbf{S}}(\mathbf{r} ; \mathbf{R}, \xi)\rangle_{\mathbf{R}, \xi}=\int_{4 \pi} \mathrm{d} \hat{\mathbf{q}} \hat{\mathbf{q}} \widetilde{I}(\mathbf{r}, \hat{\mathbf{q}})$,

where the integration is performed over all directions of the unit vector $\hat{\mathbf{q}}$ and $\widetilde{I}(\mathbf{r}, \hat{\mathbf{q}})$ is the first element of the 4element column

$\widetilde{\mathbf{I}}(\mathbf{r}, \hat{\mathbf{q}})=\left[\begin{array}{c}\widetilde{I}(\mathbf{r}, \hat{\mathbf{q}}) \\ \widetilde{Q}(\mathbf{r}, \hat{\mathbf{q}}) \\ \widetilde{U}(\mathbf{r}, \hat{\mathbf{q}}) \\ \widetilde{V}(\mathbf{r}, \hat{\mathbf{q}})\end{array}\right]$.

The latter is the solution of the following matrix integrodifferential equation:

$\hat{\mathbf{q}} \cdot \nabla \widetilde{\mathbf{I}}(\mathbf{r}, \hat{\mathbf{q}})=-n_{0}\langle\mathbf{K}(\hat{\mathbf{q}} ; \xi)\rangle_{\xi} \widetilde{\mathbf{I}}(\mathbf{r}, \hat{\mathbf{q}})+n_{0} \int_{4 \pi} \mathrm{d} \hat{\mathbf{q}}^{\prime}\left\langle\mathbf{Z}\left(\hat{\mathbf{q}}, \hat{\mathbf{q}}^{\prime} ; \xi\right)\right\rangle_{\xi} \widetilde{\mathbf{I}}\left(\mathbf{r}, \hat{\mathbf{q}}^{\prime}\right)$,

where $n_{0}$ is the average number of particles per unit volume, $\langle\mathbf{K}(\hat{\mathbf{q}} ; \xi)\rangle_{\xi}$ is the $4 \times 4$ real-valued single-particle extinction matrix averaged over the microphysical states of all the $N$ particles, and $\left\langle\mathbf{Z}\left(\hat{\mathbf{q}}, \hat{\mathbf{q}}^{\prime} ; \xi\right)\right\rangle_{\xi}$ is the $4 \times 4$ real-valued singleparticle phase matrix, also averaged over the microphysical states of all the $N$ particles. The elements of the matrices $\mathbf{K}(\hat{\mathbf{q}} ; \xi)$ and $\mathbf{Z}\left(\hat{\mathbf{q}}, \hat{\mathbf{q}}^{\prime} ; \xi\right)$ are expressed in the elements of the 
so-called $2 \times 2$ amplitude scattering matrix describing farfield scattering of a plane electromagnetic wave by an isolated particle with microphysical characteristics described by the state $\xi[139,140]$.

I provide no details of the derivation leading to Eq. (44) because this derivation contains no new concepts and is a straightforward, albeit nontrivial and instructive, mathematical exercise. It is worth noting, however, that the angular argument $\hat{\mathbf{q}}$ of $\widetilde{I}(\mathbf{r}, \hat{\mathbf{q}})$ (as well as of $\widetilde{\mathbf{I}}(\mathbf{r}, \hat{\mathbf{q}})$ ) at an internal point $\mathbf{r}$ spans the entire range of directions, $\hat{\mathbf{q}} \in 4 \pi$, since for any $\hat{\mathbf{q}}$ there is always a contribution to $\langle\widetilde{\mathbf{S}}(\mathbf{r} ; \mathbf{R}, \xi)\rangle_{\mathbf{R}, \xi}$ from pairs of multi-particle sequences such that the left-most common particle of each pair is located in the direction $-\hat{\mathbf{q}}$ relative to $\mathbf{r}$.

\subsection{Time-averaged reading of a WCR}

Consider now a WCR placed inside the random particulate medium, as shown in Fig. 21. The discussion in Section 3 implies that in the framework of the far-field Neumann expansion (39), this instrument reacts only to partial wavelets generated by multi-particle sequences having their end particles located within the conical acceptance volume $\Delta V_{\hat{\mathbf{q}}}$ defined by the WCR's small acceptance solid angle $\Delta \Omega_{\hat{\mathbf{q}}}$. Thus, the reading of the instrument is defined not by the full time-averaged PST, but rather by the corresponding partial PST $\left\langle\stackrel{\leftrightarrow}{P}\left(\mathbf{r} ; \Delta V_{\hat{\mathbf{q}}}\right)\right\rangle_{\mathbf{R}, \xi}$, where the subscripts $\mathbf{R}$ and $\xi$ denote averaging over coordinates and states of all the $N$ particles constituting the medium and not just those located inside $\Delta V_{\hat{\mathbf{q}}} \cdot\left\langle\stackrel{\leftrightarrow}{P}\left(\mathbf{r} ; \Delta V_{\hat{\mathbf{q}}}\right)\right\rangle_{\mathbf{R}, \xi}$ can be computed by making the standard assumptions invoked previously to calculate $\langle\stackrel{\leftrightarrow}{P}(\mathbf{r})\rangle_{\mathbf{R}, \xi}$, but also requiring that the end particle of any multi-particle sequence be located inside the acceptance volume $\Delta V_{\hat{\mathbf{q}}}$. This lengthy yet straightforward computation [140] shows that the reading of a WCR shown in Fig. 21 is given by

$\langle\langle$ EM power $(\mathbf{r}, \hat{\mathbf{q}})\rangle\rangle \approx S_{0} \int_{\Delta \Omega_{\hat{\mathbf{q}}}} \mathrm{d} \hat{\mathbf{q}} \widetilde{I}\left(\mathbf{r}, \hat{\mathbf{q}}^{\prime}\right)$,

where the unit vector $\hat{\mathbf{q}}$ specifies the orientation of the optical axis of the WCR and $S_{\mathrm{o}}$ is the surface area of the objective lens. If the WCR can measure all four Stokes parameters, then its polarized reading per unit time is given by

$\langle\langle\operatorname{Signal}(\mathbf{r}, \hat{\mathbf{q}})\rangle\rangle \approx S_{0} \int_{\Delta \Omega_{\hat{\mathbf{q}}}} \mathrm{d} \hat{\mathbf{q}} \widetilde{\mathbf{I}}\left(\mathbf{r}, \hat{\mathbf{q}}^{\prime}\right)$.

The fact that the signal recorded by the WCR can be modeled theoretically by solving the RTE often makes the \{WCR, RTE\} combination a useful optical-characterization tool. Furthermore, comparison of Eqs. (44) and (47) reveals that a WCR can be used to solve the energy-budget problem experimentally by integrating its reading over the entire range of WCR's orientations $\hat{\mathbf{q}} \in 4 \pi$. Of course, to make such optical-characterization and energy-budget applications of WCRs possible, the random particulate medium must possess the specific macro- and microphysical properties discussed earlier.

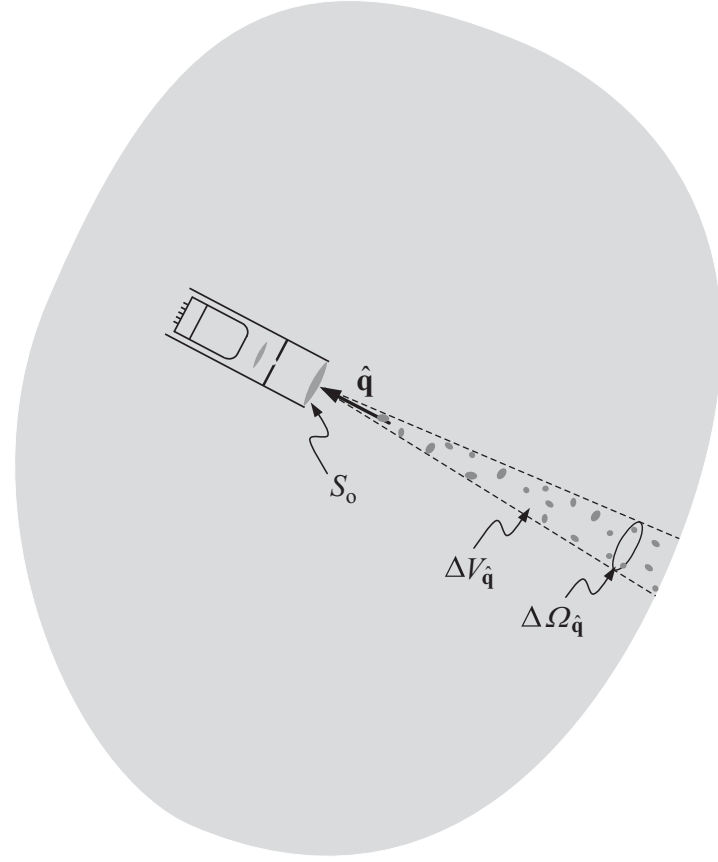

Fig. 21. A WCR placed inside the random particulate medium.

4.11. Corollaries of the microphysical approach to directional radiometry and radiative transfer

Eqs. (44)-(48) can easily be generalized to include the case of an external observation point [140] and finalize the solution of the two key problems formulated at the beginning of Section 4. The implications of the direct derivation of these formulas from the frequency-domain MMEs are quite profound and can be formulated as follows [140].

1. The derivation of Eqs. (44)-(48) does not need fundamental physical laws other than the MMEs. In particular, the ill-defined concepts of independently scattering particles, collective effects, elementary volume elements, incoherent light rays, and photons as localized particles of light have no relevance whatsoever to the transport of electromagnetic radiation in elastically scattering particulate media. Although Eq. (46) (traditionally called the RTE) has the formal mathematical structure of a kinetic equation describing the transport of point-like particles [189], it follows directly from the electromagnetic wave theory.

2. The 4-element column (45) (traditionally called the specific intensity column vector) does not characterize the instantaneous distribution of the radiation field inside the particulate medium. Instead, it emerges as a result of averaging over a sufficiently long period of time. The minimal averaging time necessary to ensure statistical ergodicity may vary depending on the particulate medium in question, but the following is always true: the longer the averaging time the more accurate the theoretical prediction based on Eqs. (44), (47), and (48). The accumulation of a signal over an extended time interval is often used to improve the 
measurement accuracy by suppressing the effect of random noise. However, the situation with Eqs. (44)(48) is fundamentally different since the very applicability of these formulas relies upon averaging over a sufficiently long period of time.

3. To ensure the applicability of Eqs. (44)-(48), the overall size of the particulate medium must be much greater than the wavelength, the average particle size, and the average distance between two neighboring particles.

4. In the context of Eqs. (44)-(48), each particle with its individual extinction and phase matrices is effectively replaced with a virtual random particle characterized by the extinction and phase matrices obtained by averaging over the microphysical states of all the particles.

5. The RTE is an inherently matrix equation involving all four elements of the specific intensity column vector. Its frequently used scalar version is obtained by artificially replacing $\widetilde{\mathbf{I}}(\mathbf{r}, \hat{\mathbf{q}})$ with its first element $\widetilde{I}(\mathbf{r}, \hat{\mathbf{q}})$ (traditionally called the specific intensity or radiance) and the extinction and phase matrices with their respective $(1,1)$ elements. As such, the scalar approximation has no fundamental physical justification.

6. All four elements of the specific intensity column vector are real-valued quantities. Furthermore, the specific intensity is always nonnegative. These corollaries ensure that Eqs. (44), (47), and (48) are physically meaningful.

7. Eqs. (44), (47), and (48) are easily generalized to the case of an incident field in the form of a superposition of several polychromatic parallel beams with quasimonochromatic components and arbitrary propagation directions.

8. The quantity $\widetilde{I}(\mathbf{r}, \hat{\mathbf{q}})$ is nothing but a formal solution of the intermediate Eq. (46) and appears merely as a byproduct of the mathematical derivation of Eqs. (44) and (47) from the frequency-domain MMEs for a sparse random particulate medium. As such, it cannot be interpreted as describing the angular distribution of electromagnetic energy flow at the point r. Fundamental physical significance can be ascribed only to the integral of $\hat{\mathbf{q}} \widetilde{I}(\mathbf{r}, \hat{\mathbf{q}})$ over all directions $\hat{\mathbf{q}}$, Eq. (44), rather than to the values of $\widetilde{I}(\mathbf{r}, \hat{\mathbf{q}})$ corresponding to individual directions. For example, adding to $\widetilde{I}(\mathbf{r}, \hat{\mathbf{q}})$ any function $f(\mathbf{r}, \hat{\mathbf{q}})$ such that

$$
\int_{4 \pi} \operatorname{d} \hat{\mathbf{q}} \hat{\mathbf{q}} f(\mathbf{r}, \hat{\mathbf{q}})=\mathbf{0}
$$

yields another "specific intensity" causing the same $\langle\langle\mathbf{S}(\mathbf{r}, t)\rangle\rangle$, a simple example being any symmetric function such that $f(\mathbf{r},-\hat{\mathbf{q}})=f(\mathbf{r}, \hat{\mathbf{q}})$. We have seen before that even the Poynting vector cannot be legitimately claimed to specify the direction of timeaveraged electromagnetic energy flow; obviously, there is even less rationale for attributing any "directional energy flow" content to the specific intensity.

9. If the particles forming the scattering medium are nonabsorbing, then it follows from the RTE that the time-averaged Poynting vector is divergence free:

$$
\nabla \cdot\langle\langle\mathbf{S}(\mathbf{r}, t)\rangle\rangle=0 \text {. }
$$

This means that the time-averaged amount of electromagnetic energy entering a differential volume element per unit time is equal to the time-averaged amount of electromagnetic energy leaving the differential volume element per unit time. Since the RTE follows from the frequency-domain MMEs only upon making several well-defined assumptions, including the consideration of only the ladder diagrams, Eq. (49) shows that these assumptions are sufficiently consistent with each other in that the final result complies with the energy conservation law. Furthermore, it implies that the contribution of all the other types of diagram to the time-averaged Poynting vector must also be divergence free in the case of nonabsorbing particles.

10. Electromagnetic scattering can be caused not only by particles with distinct boundaries, but also by density and anisotropy fluctuations in rarified molecular media such as gases. Eqs. (44)-(48) remain valid in the case of scattering by a pure gaseous medium or a gaseous medium containing randomly distributed particles provided that all density/anisotropy fluctuations and particles are located in the far zones of each other.

11. It is straightforward to generalize the theory of electromagnetic scattering and the microphysical derivation of Eqs. (44)-(48) in order to account for absorption in the host medium [190-192].

12. The ladder approximation can be expected to work well in the near zone of a sparse particulate medium. In the far zone of the medium as a whole, the so-called maximally crossed (or cyclical) diagrams exemplified by Fig. 20 e must be taken into account $[178,193]$ since they cause the effect of weak localization of electromagnetic waves in the backscattering direction $[127,139,193-200]$.

\section{Concluding remarks}

Fig. 22 shows a diagram summarizing Section 4 and tracing the place of the microphysical theory of radiative transfer in particulate media within the broader context of classical Maxwell's electromagnetics. Although I have been using the adjective "microphysical" to emphasize analytical back-traceability to the MMEs, it can also be said that this theory, as well as the theory of weak localization, is part of mesoscopic physics in that it deals with a size regime that is intermediate between the microscopic and macroscopic and is characteristic of a region where a large number of particles can interact in a correlated fashion. Direct computer solutions of the MMEs described in Refs. [201,202] demonstrate indeed how the macroscopic regime of radiative transfer and weak localization emerges from the microscopic particle-level regime of Maxwell's electromagnetics upon averaging over random realizations of a multi-particle group. Instructive 


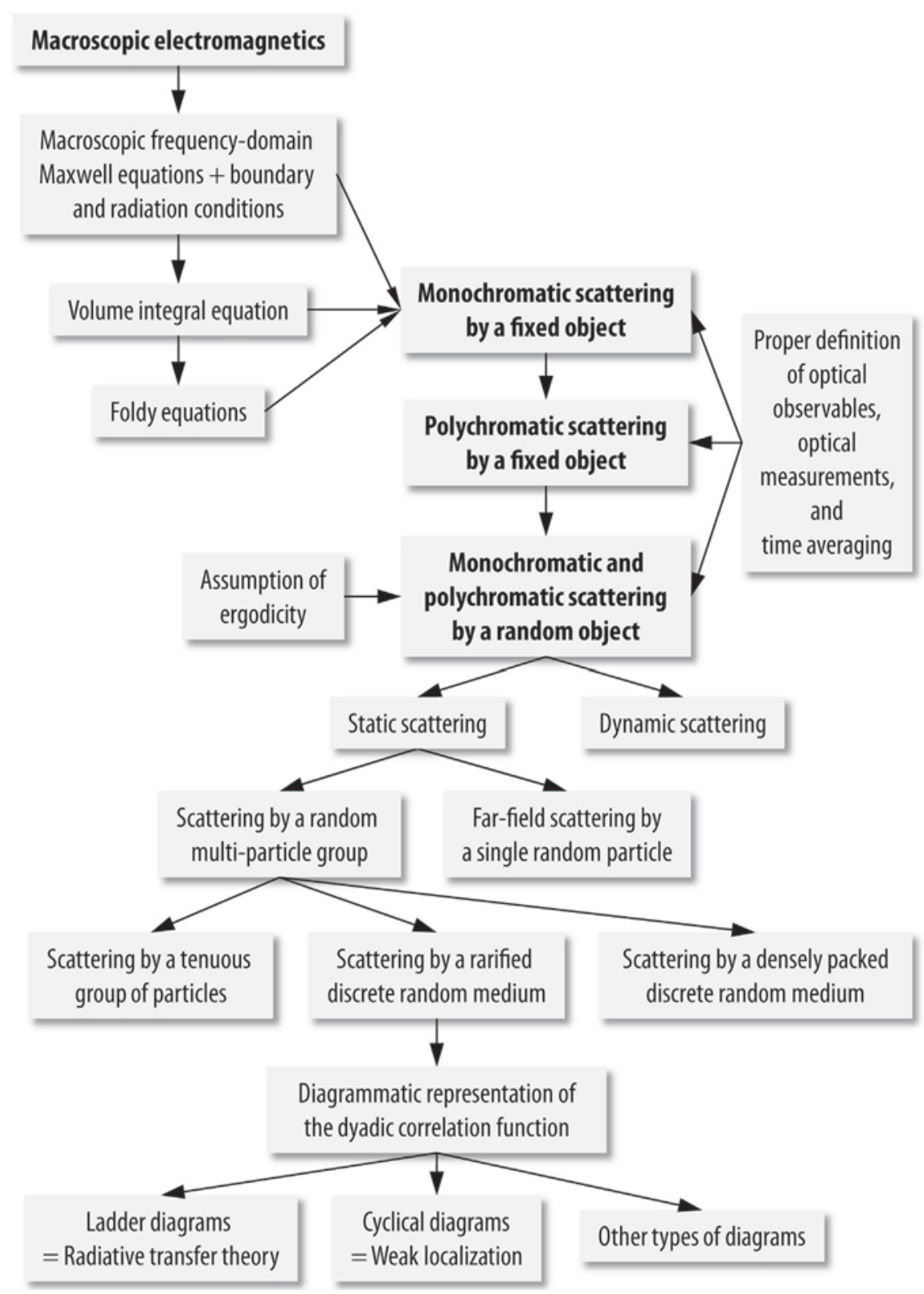

Fig. 22. Classification of electromagnetic scattering problems [140].

discussions of mesoscopic optical phenomena can be found in Refs. [199,200].

It is imperative to keep in mind that the formal applicability of the microphysical theory of radiative transfer rests on the specific approximations discussed in the preceding section, including the assumption that the scattering particles are located in each-other's far zones and are uncorrelated. The violation of this assumption in the case of densely packed particles can lead to significant errors in numerical predictions based on the RTT. Therefore, it is important to examine to what extent the RTT can be applied to densely packed particulate media. Initial results based on numerically exact solutions of the MMEs and controlled laboratory experiments have been reported in Refs. [202-205].
There is no doubt that the developments summarized in Section 4 amount to a profound paradigm shift. They reveal the inherently tensorial nature of electromagnetic energy transport in particulate media, clarify the statistical-optics content of the RTT and the physical nature of measurements with actual directional radiometers, and establish the microphysical disciplines of directional radiometry and radiative transfer in particulate media as valid and well-defined branches of physical optics. Of course, an interesting question is why these developments and their recognition by the scientific community have been so slow.

Part of the answer is provided by the following quote from Born and Wolf's Principles of Optics [118]: 
It seems to be a characteristic of the human mind that familiar concepts are abandoned only with the greatest reluctance, especially when a concrete picture of the phenomena has to be sacrificed.

Born and Wolf refer to the long struggle that Maxwell's electromagnetic theory had to endure before it had become a generally accepted fundamental paradigm. ${ }^{10}$ However, the continued unwillingness to give up the intuitively appealing yet heuristic and even unphysical concepts discussed in Section 3 shows that the above quote has much broader relevance.

The existence of the microphysical theory of radiative transfer and directional radiometry in sparse particulate media does not necessarily imply that the corresponding phenomenological disciplines will fall out of circulation any time soon. In 1965, Rudolph Preisendorfer predicted that even if a bridge connecting the mainland of fundamental physics and the island of the phenomenological RTT were built, one should not expect to see much traffic across this bridge [28]. To understand why this prediction has proved to be prophetic, one can open the standard graduate-level textbook on radiative heat transfer published in 2013 [61]. The third edition of this popular text, advertised as "a comprehensive reference for scientists, engineers, and graduate students", was "updated to include significant advances and the emergence of new research topics over the past decade". However, even though various elements of the microphysical RTT have been known for half a century, they are nowhere mentioned. Furthermore, this textbook has only one chapter on the electromagnetic wave theory which, according to the Preface, "can (and will be) skipped by most instructors for a first course in radiative heat transfer".

All in all, the resilience of the phenomenological paradigms of directional radiometry and radiative transfer has been quite enigmatic and represents an instructive proving ground for those applying the principles summarized in Thomas Kuhn's Structure [27] to explain the direction and rate of scientific progress as well as illustrate concepts such as "paradigm paralysis". In fact, it might even be of special interest to a new generation of philosophers who go far beyond the scope of Ref. [27] in exploring the social character of scientific knowledge and scientific inquiry [206].

\section{Acknowledgments}

I appreciate numerous illuminating discussions with Anatoli Borovoi, Oleg Bugaenko, Brian Cairns, Helmut Domke, Joop Hovenier, Vsevolod Ivanov, Michael Kahnert, Nikolai Khlebtsov, Pavel Litvinov, Daniel Mackowski, M. Pinar Mengüç, Viktor Tishkovets, Larry Travis, Cornelis van der Mee, Ping Yang, and Edgard Yanovitskij. Brian Cairns, Joop Hovenier, Michael Kahnert, Pavel Litvinov, Daniel Mackowski, M. Pinar Mengüç, Larry Travis, and an anonymous referee provided encouraging and helpful comments on a preliminary version of this Essay, while Lilly Del Valle helped with graphics. My research has been

\footnotetext{
${ }^{10}$ For example, Lord Kelvin, who died in 1907, had never accepted Maxwell's theory of electromagnetism.
}

partially funded over the years by the National Academy of Sciences of Ukraine, the NASA Radiation Sciences Program managed by Hal Maring, and the NASA Remote Sensing Theory Program managed by Lucia Tsaoussi.

\section{References}

[1] Hovenier JW. The polarization of light scattered by small particles: a personal review. J Quant Spectrosc Radiat Transf 2012;113: 2280-91.

[2] Van de Hulst HC. Light scattering by small particles. New York: Wiley; 1957.

[3] Van de Hulst HC. Multiple light scattering. New York: Academic Press; 1980.

[4] Hovenier JW, editor. Light scattering by non-spherical particles. J Quant Spectrosc Radiat Transf 1996;55:535-694.

[5] Van de Hulst HC. A new look at multiple scattering. New York: NASA Goddard Institute for Space Studies; 1963.

[6] Van de Hulst HC. Hints from history: a foreword. In: Mishchenko MI, Hovenier JW, Travis LD, editors. Light scattering by nonspherical particles: theory, measurements, and applications. San Diego: Academic Press; 2000. p. xxv-X.

[7] Mishchenko MI, Hovenier JW, Travis LD, editors. Light scattering by nonspherical particles: theory, measurements, and applications. San Diego: Academic Press; 2000.

[8] Hovenier JW, van der Mee CVM. Fundamental relationships relevant to the transfer of polarized light in a scattering atmosphere. Astron Astrophys 1983;128:1-16.

[9] Sobolev VV. Light scattering in planetary atmospheres. Moscow: Nauka; 1972 (in Russian). English edition: Oxford: Pergamon Press; 1975.

[10] Hansen JE, Travis LD. Light scattering in planetary atmospheres. Space Sci Rev 1974;16:527-610.

[11] Sobolev VV. Transport of radiant energy in stellar and planetary atmospheres. Moscow: Gostekhizdat; 1956 (in Russian). English edition: A treatise on radiative transfer. Princeton, NJ: Van Nostrand; 1963.

[12] Ishimaru A. Wave propagation and scattering in random media. New York: Academic Press; 1978.

[13] Lambert JH. Photometry, or on the measure and gradations of light, colors and shade. New York: Illuminating Engineering Society of North America; 2001 (English translation by DL DiLaura of the Latin original published in 1760).

[14] Kepler J. Ad Vitellionem Paralipomena, Quibus Astronomiæ pars Optica Traditur.Frankfurt: Claudium Marnium \& Hæredes Ioannis Aubrii; 1604.

[15] Bouguer P. Essai d'Optique sur la Gradation de la Lumiere. Paris: Claude Jombert; 1729.

[16] Bouguer P. Traité d'Optique sur la Gradation de la Lumiere. Paris: Académie Royale des Sciences; 1760 (English translation by WEK Middleton: Pierre Bouguer's optical treatise on the gradation of light. Toronto: University of Toronto Press; 1961).

[17] Lambert JH. Photometria, sive de Mensura et Gradibus Luminis, Colorum et Umbrae. Augsburg: Detlefsen; 1760.

[18] Beer A. Grundriss des Photometrischen Calcüles. Braunschweig: Friedrich Vieweg und Sohn; 1854.

[19] Smith DW. Phenomenology. In: Zalta EN, editor. Stanford encyclopedia of philosophy. Stanford, CA: Stanford University; 2013. 〈http://plato.stanford.edu/entries/phenomenology〉.

[20] Lambert JH. Neues Organon oder Gedanken über die Erforschung und Bezeichnung des Wahren und dessen Unterscheidung vom Irrthum und Schein. Leipzig: Johann Wendler; 1764.

[21] Gershun AA. The light field. Leningrad and Moscow: ONTI; 1936 (in Russian, English translation by Moon P, Timoshenko G. J Phys Math 1939; 18:51-151).

[22] Poincaré H. The foundations of science. Lancaster, PA: The Science Press; 1913.

[23] Bachelard G. The new scientific spirit. Boston: Beacon Press; 1984 (Original French edition: Le nouvel esprit scientifique. Paris: Presses Universitaires de France; 1934).

[24] Bachelard G. The formation of the scientific mind. Manchester: Clinamen Press; 2002 (Original French edition: La formation de l'esprit scientifique. Paris: Librairie Philosophique; 1938).

[25] Foucault M. The order of things. New York: Vintage; 1973 (Original French edition: Les Mots et les Choses. Paris: Gallimard; 1966). 
[26] Foucault M. The archaeology of knowledge. New York: Harper and Row; 1972 (Original French edition: L'Archéologie du Savoir. Paris: Gallimard; 1969).

[27] Kuhn TS. The structure of scientific revolutions. Chicago: University of Chicago Press; 1970.

[28] Preisendorfer RW. Radiative transfer on discrete spaces. Oxford: Pergamon Press; 1965.

[29] Lommel E. Die Photometrie der diffusen Zurückwerfung. Sitzber Acad Wissensch München 1887;17:95-124.

[30] Chwolson O. Grundzüge einer mathematischen Theorie der inneren Diffusion des Lichtes. Bull Acad Imp Sci St Pétersb 1889:33: 221-56.

[31] Ivanov VV. Making of radiative transfer theory. Trudy (Proc) Astron Observ St Petersb Univ 1994;44:6-29 (in Russian).

[32] Schuster A. Radiation through a foggy atmosphere. Astrophys J $1905 ; 21: 1-22$

[33] King LV. On the scattering and absorption of light in gaseous media, with applications to the intensity of sky radiation. Phil Trans R Soc Lond A 1913;212:375-433.

[34] Planck M. Theorie der Wärmestrahlung. Leipzig: Verlag Von Johann Ambrosius Barth; 1906.

[35] Planck M. The theory of heat radiation. Philadelphia: P. Blakiston's Son \& Co; 1914.

[36] Milne E. Thermodynamics of the stars. Handb Astrophys 1930;3 (Part 1):65-255

[37] Hopf E. Mathematical problems of radiative equilibrium. Cambridge, UK: Cambridge University Press; 1934.

[38] Chandrasekhar S. Radiative transfer. Oxford: Oxford University Press; 1950.

[39] Ambartsumian VA, editor. Theoretical astrophysics. London: Pergamon Press: 1958.

[40] Jerlov NG. Marine optics. Amsterdam: Elsevier; 1976.

[41] Lenoble J, editor. Radiative transfer in scattering and absorbing atmospheres: standard computational procedures. Hampton, VA: A. Deepak Publishing; 1985.

[42] Goody RM, Yung YL. Atmospheric radiation, theoretical basis. Oxford: Oxford University Press; 1989.

[43] Zege EP, Ivanov AP, Katsev IL. Image transfer through a scattering medium. Berlin: Springer; 1991.

[44] Liou KN. Radiation and cloud processes in the atmosphere: theory, observation, and modeling. New York: Oxford University Press; 1992.

[45] Lenoble J. Atmospheric radiative transfer. Hampton, VA: A. Deepak Publishing; 1993.

[46] Kirk JTO. Light and photosynthesis in aquatic ecosystems. Cambridge, UK: Cambridge University Press; 1994.

[47] McCluney WR. Introduction to radiometry and photometry. Boston, MA: Artech House; 1994.

[48] Mobley CD. Light and water: radiative transfer in natural waters. San Diego: Academic Press; 1994.

[49] Yanovitskij EG. Light scattering in inhomogeneous atmospheres. Berlin: Springer; 1997.

[50] Thomas GE, Stamnes K. Radiative transfer in the atmosphere and ocean. Cambridge, UK: Cambridge University Press; 1999.

[51] Liou KN. An introduction to atmospheric radiation. San Diego: Academic Press; 2002

[52] Hovenier JW, van der Mee C, Domke H. Transfer of polarized light in planetary atmospheres. Dordrecht: Kluwer; 2004.

[53] Kokhanovsky A. Light scattering media optics. Berlin: Springer; 2004

[54] Marshak A, Davis AB, editors. 3D radiative transfer in cloudy atmospheres. Berlin: Springer; 2005.

[55] Parr AC, Datla RU, Gardner JL, editors. Optical radiometry. Amsterdam: Elsevier; 2005.

[56] Zdunkovski W, Trautmann T, Bott A. Radiation in the atmosphere. Cambridge, UK: Cambridge University Press; 2007.

[57] Dombrovsky LA, Baillis D. Thermal radiation in disperse systems: an engineering approach. Begell House; 2010.

[58] Howell JR, Siegel R, Mengüç MP. Thermal radiation heat transfer. Boca Raton, FL: CRC Press; 2011.

[59] Bukshtab M. Applied photometry, radiometry, and measurements of optical lossess. Dordrecht: Springer; 2012.

[60] Wendisch M, Yang P. Theory of atmospheric radiative transfer. Weinheim: Wiley-VCH; 2012.

[61] Modest MF. Radiative heat transfer. San Diego: Academic Press; 2013

[62] Gans R. Die Farbe des Meeres. Ann Phys 1924;75:1-22.

[63] Rozenberg GV. Stokes vector-parameter. Uspekhi Fiz Nauk 1955;56 (1):77-110 (in Russian).
[64] Rozenberg GV. The light ray (contribution to the theory of the light field). Sov Phys Uspekhi 1977;20:55-79.

[65] Tsang L. Thermal emission of nonspherical particles. Radio Sci 1984;19:966-74.

[66] Tsang L, Kong JA, Ding K-H. Scattering of electromagnetic waves: theories and applications. New York: Wiley; 2000.

[67] Vladimirov VS. Mathematical problems in the one-velocity theory of particle transport. Chalk River, Ontario, Canada: Atomic Energy of Canada Limited; 1963. 〈http://www.iaea.org/inis/collection/ NCLCollectionStore/_Public/43/103/43103493.pdf $\rangle$.

[68] Case KM, Zweifel PF. Linear transport theory. Reading, MA: Addison-Wesley; 1967.

[69] Maslennikov MV. The Milne problem with anisotropic scattering. Providence, RI: American Mathematical Society; 1969.

[70] Kaper HG, Lekkerkerker CG, Hejtmanek J. Spectral methods in linear transport theory. Basel: Birkhäuser; 1982

[71] Ershov Yu I, Shikhov SB. Mathematical foundations of transport theory. Moscow: Energoatomizdat; 1985 (in Russian).

[72] Germogenova TA. Local properties of solutions of the transport equation. Moscow: Nauka; 1986 (in Russian).

[73] Greenberg W, van der Mee C, Protopopescu V. Boundary value problems in abstract kinetic theory. Basel: Birkhäuser; 1987.

[74] Maslennikov MV. Axiomatic model of particle transport phenomena. Moscow: Nauka; 1989 (in Russian).

[75] Agoshkov V. Boundary value problems for transport equations. Boston: Birkhäuser; 1998

[76] Dutré P, Bala K, Bekaert P. Advanced global illumination. Wellesley, MA: A. K. Peters, Ltd.; 2006.

[77] Pharr M, Humphreys G. Physically based rendering: from theory to implementation. Amsterdam: Elsevier; 2010.

[78] Lessig Ch. Modern foundations of light transport simulation. Toronto: University of Toronto; 2012. 〈http://users.cms.caltech. edu/ lessig/dissertation/lessig_dissertation.pdf $\rangle$.

[79] Maxwell JC. A dynamical theory of the electromagnetic field. Phil Trans R Soc Lond 1865:155:459-512.

[80] Stratton JA. Electromagnetic theory. New York: McGraw Hill; 1941.

[81] Jackson JD. Classical electrodynamics. New York: Wiley; 1999.

[82] Power EA. Introductory quantum electrodynamics. London: Longmans; 1964.

[83] Akhiezer AI, Berestetskii VB. Quantum electrodynamics. New York: Wiley; 1965.

[84] Healy WP. Non-relativistic quantum electrodynamics. London: Academic Press; 1982.

[85] Craig DP, Thirunamachandran T. Molecular quantum electrodynamics: an introduction to radiation-molecule interactions. London: Academic Press: 1984

[86] Cohen-Tannoudji C, Dupont-Roc J, Grynberg G. Photons and atoms: introduction to quantum electrodynamics. New York: Wiley; 1989

[87] Cohen-Tannoudji C, Dupont-Roc J, Grynberg G. Atom-photon interactions: basic processes and applications. New York: Wiley; 1992.

[88] Weinberg S. The quantum theory of fields, vol. 1. Cambridge, UK: Cambridge University Press; 1995.

[89] Ripoll J. Derivation of the scalar radiative transfer equation from energy conservation of Maxwell's equations in the far field. J Opt Soc Am A 2011:28:1765-75.

[90] Lorentz HA. The theory of electrons. Leipzig: B. G. Teubner; 1916.

[91] Arons AB, Peppard MB. Einstein's proposal of the photon concept a translation of the Annalen der Physik paper of 1905. Am J Phys 1965;33:367-74.

[92] Pomraning GC. The equations of radiation hydrodynamics. Oxford: Pergamon Press; 1973.

[93] Mihalas D, Weibel-Mihalas B. Foundations of radiation hydrodynamics. Oxford: Oxford University Press; 1984.

[94] Oxenius J. Kinetic theory of particles and photons. Berlin: Springer: 1986.

[95] Pomraning GC. Linear kinetic theory and particle transport in stochastic mixtures. Singapore: World Scientific; 1991.

[96] Otto S. Analytical properties of the radiance in atmospheric radiative transfer theory. J Quant Spectrosc Radiat Transf 2014;133: 329-50.

[97] Taylor JR, Zafiratos CD, Dubson MA. Modern physics for scientists and engineers. Upper Saddle River, NJ: Prentice Hall; 2004.

[98] Wentzel G. Zur Theorie des photoelektrischen Effekts. Z Phys 1926;40:574-89.

[99] Dodd JN. Atoms and light: interactions. New York: Plenum Press; 1991.

[100] Fearn H, Lamb Jr. WE. Corrections to the golden rule. Phys Rev A $1991 ; 43: 2124-8$. 
[101] Kimble HJ, Mandel L. Photoelectric detection of polychromatic light. Phys Rev A 1984;30:844-50.

[102] Petty GW. A first course in atmospheric radiation. Madison, WI: Sundog Publishing; 2006.

[103] Bohren CF, Clothiaux EE. Fundamentals of atmospheric radiation. Weinheim: Wiley-VCH; 2006.

[104] Scully MO, Sargent M. III. The concept of the photon. Phys Today 1972(3):38-47.

[105] Kidd R, Ardini J, Anton A. Evolution of the modern photon. Am J Phys 1989;57:27-35.

[106] Lamb Jr. WE. Anti-photon. Appl Phys B 1995;60:77-84.

[107] Bohm D. Quantum theory. Englewood Cliffs, NJ: Prentice-Hall; 1951.

[108] Kramers HA. Quantum mechanics. Amsterdam: North-Holland; 1957.

[109] Mandel L, Wolf E. Optical coherence and quantum optics. Cambridge, UK: Cambridge University Press; 1995.

[110] Meystre P, Sargent III M. Elements of quantum optics. Berlin: Springer; 1999.

[111] Wolf E. Coherence and radiometry. J Opt Soc Am 1978;68:6-17.

[112] Dirac PAM. The quantum theory of the emission and absorption of radiation. Proc R Soc Lond A 1927; 114:243-50.

[113] Jordan P, Pauli W. Zur Quantenelektrodynamik ladungsfreier Felder. Z Phys 1928;47:151-73.

[114] Heisenberg W, Pauli W. Zur Quantendynamik der Wellenfelder. Z Phys 1929;56:1-61.

[115] Heisenberg W, Pauli W. Zur Quantentheorie der Wellenfelder. II. Z Phys 1930;59:168-90.

[116] Mishchenko MI. The Poynting-Stokes tensor and radiative transfer in discrete random media: the microphysical paradigm. Opt Express 2010;18:19770-91.

[117] Mishchenko MI. Measurement of electromagnetic energy flow through a sparse particulate medium: a perspective. J Quant Spectrosc Radiat Transf 2013;123:122-34.

[118] Born M, Wolf E. Principles of optics. Cambridge, UK: Cambridge University Press; 1999.

[119] Goodman JW. Introduction to Fourier optics. Englewood, CO: Roberts \& Company; 2005.

[120] Darecki M, Stramski D, Sokólski M. Measurements of highfrequency light fluctuations induced by sea surface waves with an Underwater Porcupine Radiometer System. J Geophys Res 2011;116:COOH09.

[121] Apresyan LA, Kravtsov Yu A. Radiation transfer. Statistical and wave aspects. Basel: Gordon and Breach; 1996.

[122] Borovoi AG. Method of iterations in multiple scattering: the transfer equation. Izv Vuzov Fiz 1966;6:50-4 (in Russian).

[123] Barabanenkov Yu N. Equation of radiative transfer in a model of isotropic point scatterers. Sov Phys Dokl 1967;12:431-3.

[124] Walther A. Radiometry and coherence. J Opt Soc Am 1968;58: 1256-9.

[125] Dolginov AZ, Gnedin Yu N, Silant'ev NA. Photon polarization and frequency change in multiple scattering. J Quant Spectrosc Radiat Transf 1970;10:707-54.

[126] Marchand EW, Wolf E. Radiometry with sources of any state of coherence. J Opt Soc Am 1974;64:1219-26.

[127] Barabanenkov Yu N. Multiple scattering of waves by ensembles of particles and the theory of radiation transport. Sov Phys Uspekhi 1975;18:673-89.

[128] Wolf E. New theory of radiative energy transfer in free electromagnetic fields. Phys Rev D 1976;13:869-86.

[129] Zubairy MS, Wolf E. Exact equations for radiative transfer of energy and momentum in free electromagnetic fields. Opt Commun 1977;20:321-4.

[130] Walther A. Theorem on the uniqueness of the generalized radiance. Opt Lett 1978;3:127-9.

[131] Fante RL. Relationship between radiative-transport theory and Maxwell's equations in dielectric media. J Opt Soc Am 1981;71: $460-8$.

[132] Sudarshan ECG. Quantum theory of radiative transfer. Phys Rev A 1981;23:2802-9.

[133] Pedersen HM. Exact geometrical theory of free-space radiative energy transfer. J Opt Soc Am A 1991;8:176-85.

[134] Friberg AT, Agarwal GS, Foley JT, Wolf E. Statistical wave-theoretical derivation of the free-space transport equation of radiometry. J Opt Soc Am B 1992;9:1386-93.

[135] Greffet J-J, Nieto-Vesperinas M. Field theory for generalized bidirectional reflectivity: derivation of Helmholtz's reciprocity principle and Kirchhoff's law. J Opt Soc Am A 1998;15:2735-44.
[136] Tsang L, Kong JA. Scattering of electromagnetic waves: advanced topics. New York: Wiley; 2001.

[137] Mishchenko MI. Vector radiative transfer equation for arbitrarily shaped and arbitrarily oriented particles: a microphysical derivation from statistical electromagnetics. Appl Opt 2002;41:7114-34.

[138] Mishchenko MI. Microphysical approach to polarized radiative transfer: extension to the case of an external observation point. Appl Opt 2003;42:4963-7.

[139] Mishchenko MI, Travis LD, Lacis AA. Multiple scattering of light by particles: radiative transfer and coherent backscattering. Cambridge, UK: Cambridge University Press; 2006. 〈http://www.giss.nasa.gov/ staff/mmishchenko/books.html>.

[140] Mishchenko MI. Electromagnetic scattering by particles and particle groups: an introduction. Cambridge, UK: Cambridge University Press; 2014.

[141] Mishchenko MI. Directional radiometry and radiative transfer: a new paradigm. J Quant Spectrosc Radiat Transf 2011;112:2079-94.

[142] Boltzmann L. Vorlesungen über Maxwells Theorie der Elektricität und des Lichtes, vol. II. Leipzig: J. A. Barth; 1893.

[143] Kline M. Mathematical thought from ancient to modern times. New York: Oxford University Press; 1972.

[144] Whittaker E. A history of the theories of aether and electricity, vols. I-II. New York: American Institute of Physics; 1987.

[145] Hunt BJ. The Maxwellians. Ithaca, NY: Cornell University Press; 1991.

[146] Maxwell JC. A treatise on electricity and magnetism. Oxford: Clarendon Press; 1873.

[147] Heaviside O. Electromagnetic theory. New York: Dover; 1950.

[148] Poincaré H. L'état actuel et l'avenir de la Physique mathématique. Bull Sci Math 1904;28: 302-24. English translation: The principles of mathematical physics. In: Rogers HJ, editor. Congress of arts and science, St. Louis, 1904, vol. I. Cambridge, MA: Riverside Press; 1905. p. 604-22.

[149] Poincaré H. Sur la dynamique de l'électron. C R Acad Sci 1905;140: 1504-8.

[150] Poincaré H. Sur la dynamique de l'électron. Rend Circ Matem Palermo 1906;21:129-75.

[151] Poynting JH. On the transfer of energy in the electromagnetic field. Phil Trans R Soc Lond 1884;175:343-61.

[152] Heaviside O. Electromagnetic induction and its propagation. Electrician 1885;14:178-80, 306-7.

[153] de Groot SR, Suttorp LG. Foundations of electrodynamics. Amsterdam: North-Holland; 1972.

[154] Robinson FNH. Macroscopic electromagnetism. Oxford: Pergamon Press; 1973.

[155] Huttner B, Barnett SM. Quantization of the electromagnetic field in dielectrics. Phys Rev A 1992;46:4306-22.

[156] Ho S-T, Kumar P. Quantum optics in a dielectric: macroscopic electromagnetic-field and medium operators for a linear dispersive lossy medium - a microscopic derivation of the operators and their commutation relations. J Opt Soc Am B 1993;10:1620-36.

[157] Suttorp LG, Wubs M. Field quantization in inhomogeneous absorptive dielectrics. Phys Rev A 2004;70:013816.

[158] Lukš A, Peřinová V. Quantum aspects of light propagation. Berlin: Springer; 2009.

[159] Huffman DR. The applicability of bulk optical constants to small particles. In: Barber PW, Chang RK, editors. Optical effects associated with small particles. Singapore: World Scientific; 1988. p. 279-324.

[160] Müller C. Foundations of the mathematical theory of electromagnetic waves. Berlin: Springer; 1969.

[161] Sommerfeld A. Die Greensche Funktion der Schwingungsgleichung. Jahresber Deutsch Math-Verein 1912;21:309-53.

[162] Schot SH. Eighty years of Sommerfeld's radiation condition. Hist Math 1992;19:385-401.

[163] Silver S, editor. Microwave antenna theory and design. New York: McGraw-Hill; 1949.

[164] Levine H, Schwinger J. On the theory of electromagnetic wave diffraction by an aperture in an infinite plane conducting screen. Commun Pure Appl Math 1950;3:355-91.

[165] Green G. An essay on the application of mathematical analysis to the theories of electricity and magnetism. Nottingham: T. Wheelhouse; 1828.

[166] Saxon DS. Lectures on the scattering of light. Los Angeles: University of California at Los Angeles; 1955 (Scientific Report No. 9, Department of Meteorology).

[167] Dmitriev VI, Zakharov EV. Integral equations in boundary problems of electrodynamics. Moscow: Moscow State University Press; 1987 (in Russian). 
[168] Mishchenko MI. Gustav Mie and the fundamental concept of electromagnetic scattering by particles: a perspective. J Quant Spectrosc Radiat Transf 2009; 110:1210-22.

[169] Lippmann BA, Schwinger J. Variational principles for scattering processes. Phys Rev 1950;79:469-80.

[170] Goldberger ML, Watson KM. Collision theory. New York: Wiley; 1964.

[171] Newton RG. Scattering theory of waves and particles. New York: Springer; 1982.

[172] Tsang L, Kong JA. Multiple scattering of electromagnetic waves by random distributions of discrete scatterers with coherent potential and quantum mechanical formalism. J Appl Phys 1980;51:3465-85.

[173] Prishivalko AP, Babenko VA, Kuzmin VN. Scattering and absorption of light by inhomogeneous and anisotropic spherical particles. Minsk: Nauka i Tekhnika; 1984 (in Russian).

[174] Tsang L, Kong JA, Shin RT. Theory of microwave remote sensing. New York: Wiley; 1985.

[175] Foldy LL. The multiple scattering of waves. Phys Rev 1945;67: 107-19.

[176] Lax M. Multiple scattering of waves. Rev Mod Phys 1951;23: 287-310.

[177] Watson KM. Multiple scattering and the many-body problem applications to photomeson production in complex nuclei. Phys Rev 1953;89:575-87.

[178] Watson KM. Multiple scattering of electromagnetic waves in an underdense plasma. J Math Phys 1969;10:688-702.

[179] Martin PA. Multiple scattering. Interaction of time-harmonic waves with $N$ obstacles. Cambridge, UK: Cambridge University Press; 2006.

[180] Mishchenko MI, Tishkovets VP, Travis LD, et al. Electromagnetic scattering by a morphologically complex object: fundamental concepts and common misconceptions. J Quant Spectrosc Radiat Transf 2011;112:671-92.

[181] Yosida K. Lectures on differential and integral equations. New York: Interscience; 1960.

[182] Arfken GB, Weber HJ. Mathematical methods for physicists. Amsterdam: Elsevier; 2005.

[183] Babenko VA, Astafyeva LG, Kuzmin VN. Electromagnetic scattering in disperse media: inhomogeneous and anisotropic particles. Chichester, UK: Praxis; 2003.

[184] Twersky V. On propagation in random media of discrete scatterers. Proc Symp Appl Math 1964;16:84-116.

[185] Poincaré H. Sur le problème des trois corps et les équations de la dynamique. Acta Math 1890;13:1-270.

[186] Khinchin AI. Mathematical foundations of statistical mechanics. New York: Dover; 1949.

[187] Uhlenbeck GE, Ford GW. Lectures in statistical mechanics. Providence, RI: American Mathematical Society; 1963.
[188] Farquhar IE. Ergodic theory in statistical mechanics. London: Wiley; 1964.

[189] Duderstadt JJ, Martin WR. Transport theory. New York: Wiley; 1979.

[190] Mishchenko MI. Electromagnetic scattering by a fixed finite object embedded in an absorbing medium. Opt Express 2007;15: 13188-202.

[191] Mishchenko MI. Multiple scattering by particles embedded in an absorbing medium. 1. Foldy-Lax equations, order-of-scattering expansion, and coherent field. Opt Express 2008;16:2288-301.

[192] Mishchenko MI. Multiple scattering by particles embedded in an absorbing medium. 2. Radiative transfer equation. J Quant Spectrosc Radiat Transf 2008;109:2386-90.

[193] Barabanenkov Yu N. Wave corrections to the transfer equation for "back" scattering. Radiophys Quantum Electron 1973;16:65-71.

[194] Kuga Y, Ishimaru A. Retroreflectance from a dense distribution of spherical particles. J Opt Soc Am A 1984;1:831-5.

[195] Tsang L, Ishimaru A. Backscattering enhancement of random discrete scatterers. J Opt Soc Am A 1984;1:836-9.

[196] Wolf P, Maret G. Weak localization and coherent backscattering of photons in disordered media. Phys Rev Lett 1985;55:2696-9.

[197] Van Albada MP, Lagendijk A. Observation of weak localization of light in a random medium. Phys Rev Lett 1985;55:2692-5.

[198] Barabanenkov Yu N, Kravtsov Yu A, Ozrin VD, Saichev AI. Enhanced backscattering in optics. Prog Opt 1991;29:65-197.

[199] Sheng P. Introduction to wave scattering, localization, and mesoscopic phenomena. Berlin: Springer; 2006.

[200] Akkermans E, Montambaux G. Mesoscopic physics of electrons and photons. Cambridge, UK: Cambridge University Press; 2007.

[201] Mishchenko MI, Liu L, Mackowski DW, Cairns B, Videen V. Multiple scattering by random particulate media: exact 3D results. Opt Express 2007;15:2822-36.

[202] Mackowski DW, Mishchenko MI. Direct simulation of extinction in a slab of spherical particles. J Quant Spectrosc Radiat Transf 2013;123:103-12.

[203] Voit F, Schäfer J, Kienle A. Light scattering by multiple spheres: comparison between Maxwell theory and radiative-transfertheory calculations. Opt Lett 2009;34:2593-5.

[204] Muinonen K, Mishchenko MI, Dlugach JM, Zubko E, Penttilä A, Videen G. Coherent backscattering verified numerically for a finite volume of spherical particles. Astrophys J 2012;760:118.

[205] Mishchenko MI, Goldstein D, Chowdhary J, Lompado A. Radiative transfer theory verified by controlled laboratory experiments. Opt Lett 2013;38:3522-5.

[206] Longino H. The social dimensions of scientific knowledge. In: Zalta EN, editor. Stanford encyclopedia of philosophy. Stanford, CA: Stanford University; 2013. 〈http://plato.stanford.edu/entries/ scientific-knowledge-social〉. 